\title{
CANCER CELL CYTOSKELETON BEHAVIOR ON TITANIUM OXIDES SYNTHESIZED THROUGH ULTRAFAST PULSED LASER IRRADIATION
}

by

Chandramouli Chinnakkannu Vijayakumar

B.Tech., SRM University, Chennai, India, 2008

M.S., SUNY Stony Brook University, New York, USA, 2010

A thesis presented to

Ryerson University in partial fulfillment of the requirements

for the degree of Master of Applied Science in the

Program of Mechanical and Industrial Engineering

Toronto, Ontario, Canada, 2015

(C) Chandramouli Chinnakkannu Vijayakumar 2015 


\section{AUTHORS DECLARATION FOR ELECTRONIC SUBMISSION OF THESIS}

I hereby declare that I am the sole author of this thesis. This is a true copy of the thesis, including any required final revisions, as accepted by my examiners.

I authorize Ryerson University to lend this thesis to other institutions or individuals for the purpose of scholarly research.

I further authorize Ryerson University to reproduce this thesis by photocopying or by other means, in total or in part, at the request of other institutions or individuals for the purpose of scholarly research.

I understand that my thesis may be made electronically available to the public. 


\title{
Cancer cell cytoskeleton behavior on titanium oxides synthesized through ultrafast pulsed laser irradiation
}

\author{
Chandramouli Chinnakkannu Vijayakumar \\ Master of Applied Science in Mechanical and Industrial \\ Engineering \\ Ryerson University, 2015
}

\begin{abstract}
Conventionally, single phases of $\mathrm{TiO}_{2}$ are used for targeted therapy and a drug carrier systems. In this research a harmonized approach in synthesizing multi-Ti oxide phases in a nanostructure and its ability to control cancer cell cytoskeleton behavior. This modulation of HeLa cancer cell cytoskeleton behaviour including shape of the cell, surface area of the cell, alignment of the cell is diligent by using the combination of $\mathrm{TiO}, \mathrm{Ti}_{3} \mathrm{O}, \mathrm{Ti}_{2} \mathrm{O}$ phases. Field emission scanning electron microscope investigation (FESEM) revealed that multi-Ti oxide nanostructure revealed a greater reduction of HeLa cell relative to fibroblast cell. This altered cell adhesion was followed by modulation of HeLa cell architecture with significant reduction in actin stress fibers. The intricate combination of multi-Ti oxide nanostructures renders a biomaterial that can precisely alter HeLa cell but not the fibroblast cell behaviour has the potential application of creating a multi-Ti oxide nanostructure for targeted cancer therapy, developing nano patterning devices. This unique interaction of HeLa cancer cell with multi-Ti oxide nanostructure has provided an insight of cellcell signalling which is the fundamental mechanism in regulating their proliferative characteristics.
\end{abstract}




\section{Acknowledgements}

I express my deepest gratitude to my supervisor Dr.Krishnan Venkatakrishnan for his guidance, understanding, patience, constant support and encouragement in every aspect of my life. His mentorship was paramount in shaping me to an independent thinker and providing constant expertise. I would also like to thank my Co-Supervisor Dr.Bo Tan for helping me to understand the physics behind laser fabrication and revising my thesis. I have been fortunate to have supervisors who had faith in me and gave me an opportunity to establish a career in Canada.

I would also like to thank my loving parents Mr.M.M.C.Vijayakumar and Mrs. Devi Vijayakumar for their constant support and motivation throughout my life. I would like to thank my brother Sivaprasad who was a provided step by step guidance throughout my education and helped me when I need of guidance.

Last but not the least I would like to thank my friends who supported me through good and bad times in my research. My sincere gratitude to interns Advait Pai who helped me to draw various illustrations and spent hours working with me patiently. Also Nasif Nayeer who did statistical analysis for my research and helped to edit my thesis by working long hours. I would especially like to thank mechanical and industrial engineering staff for their support.

Finally, I thank the almighty for paving the path for my success. 


\section{Table of Contents}

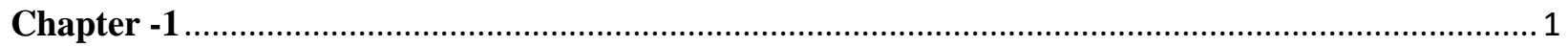

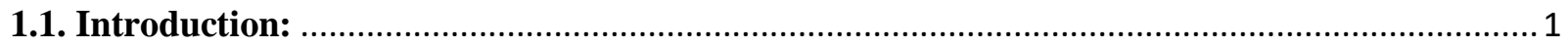

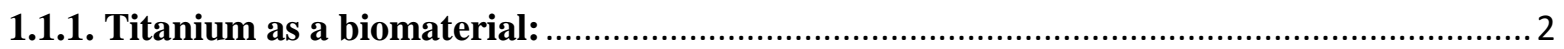

1.1.2. Methods of Fabricating Titanium Oxide Nanostructures:

1.2 Influence of various morphology of nanostructures for targeted therapy: .............................. 6

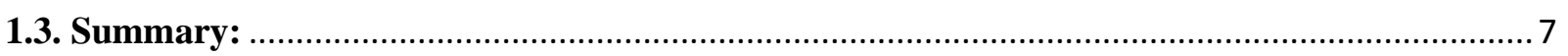

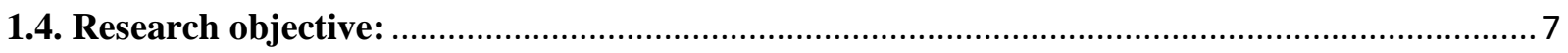

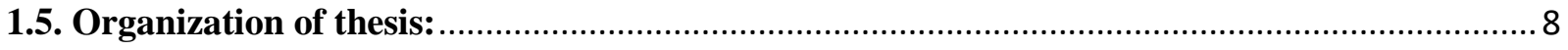

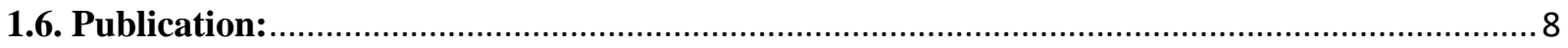

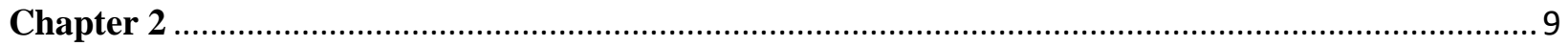

2.1 Materials and methods: ………………………………............................................

2.1.1. Synthesizing multi-Ti oxide phased nanostructure formation throughultrafast

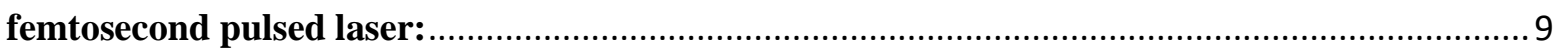

2.1.2 Cell culture:

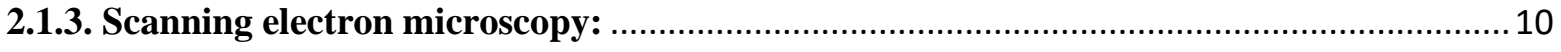

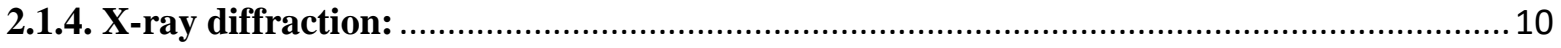

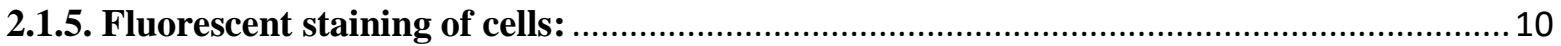

2.1.6. Measuring cellular alignment using F-actin ............................................................

2.1.7. Nanoparticle size measurement:.........................................................................11

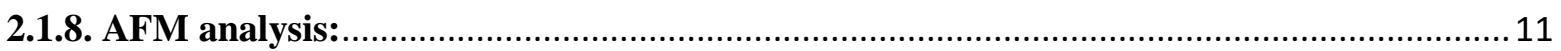

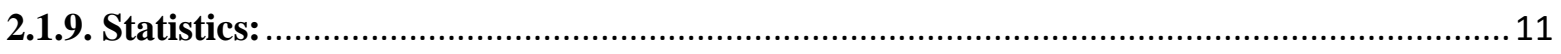

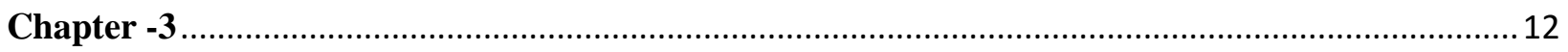

Harmonizing HeLa cell cytoskeleton behaviour by multi-Ti oxide phased nano structure

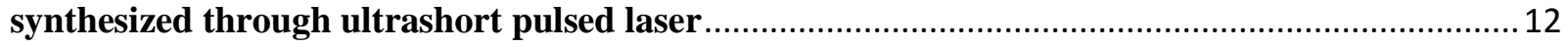

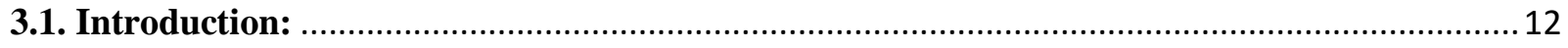

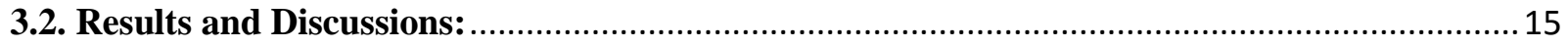

3.2.1. Synthesizing multi-Ti oxide phased nanostructures using ultrashort pulsed laser: .........15

3.2.2. Mechanism of multi-Ti oxide phased nanostructure formation by ultrashort pulsed laser:

3.2.3. FESEM and HR-TEM-EDX analysis of multi-Ti oxide phased nanostructure:..............19

3.2.4. Elemental characterization of multi-Ti oxide phased nanostructure by $\mathrm{X}$-ray diffraction

3.2.5. Decreased HeLa and NIH3T3 cell adhesion on multi-Ti oxide phased nano structure: ..22 
3.2.6. Modulating HeLa cell cytoskeleton behaviour when interacting on multi-Ti oxide phased nanostructure

3.2.7. Lack of Stress fiber on multi-Ti oxide phased nano structure: ....................................26

3.2.8. AFM analysis of multi-Ti oxide phased nanostructure interacting with HeLa cell:.........27

3.2.9. Surface area analysis of HeLa cell on multi-Ti oxide phased nano structure: .................29

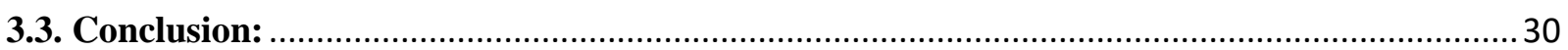

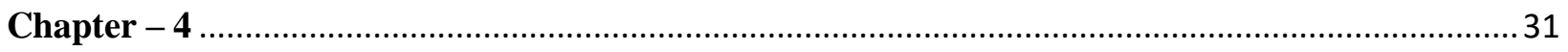

HeLa cell patterning by ying-yang proliferative zones using multiphase titanium oxide nanomaterial synthesized by ultrashort pulsed laser ................................................................ 31

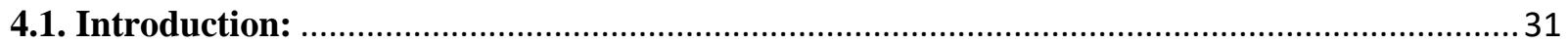

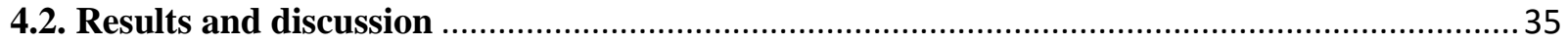

4.2.1. FESEM with EDX analysis of nanostructures generated by varying ionization energy:. 35

4.2.2. AFM analysis of investigating nanostructures generated at various ionization energy: .. 35

4.2.3. Effect of nanoparticle size by varying the ionization energy: .........................................36

4.2.4. XRD investigation of multiphase titanium oxide nanostructure generated by varying

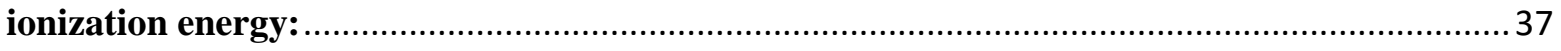

4.2.5. Decrease of HeLa cell adhesion pattern on nanostructure synthesized by varying the

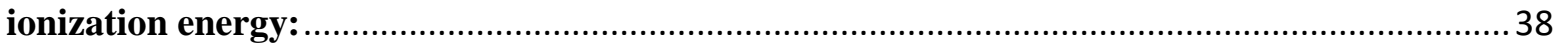

4.2.6. Proliferative and Non-proliferative zone determine the HeLa cell cytoskeleton shape.... 40

4.2.7. Various patterns of multiphase titanium oxide nanostructures determine the HeLa cell into proliferative and non-proliferative zone: ..................................................................... 42

4.3. Conclusion:

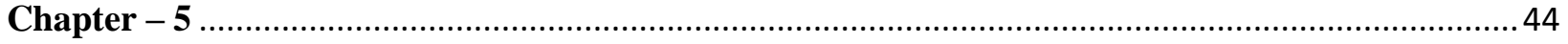

Cancer cell signalling under the influence of multi-Ti oxide nanostructures having dominant phases

5.1. Introduction: 44

5.2. Results and discussion:

5.2.1. Influence of phase dominance of titanium oxide nanostructure determines cell-to-cell signaling

5.2.1. Mechanotransduction mechanism by which cellular elongation is determined by the influence of multi-Ti oxide phased nanostructure:

5.2.2. Directed HeLa cell and NIH3T3 cell repelling away from multi-Ti oxide nanostructure:

5.2.3. Effect of multi-Ti oxide phases of nanostructure for cancer cell directionality:

5.2.4. HeLa cell alignment in the proliferative zone due to the influence of energy from the ultrashort pulsed laser: 
5.2.5. Rounding of HeLa cancer cell when interacting with multi-Ti oxide phased nanostructure:

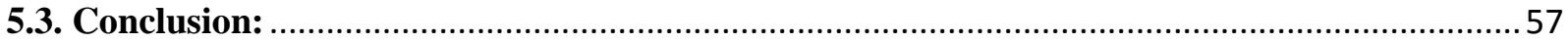

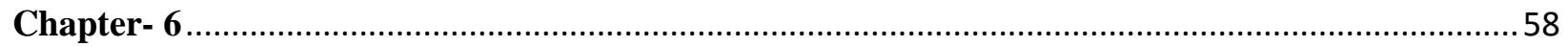

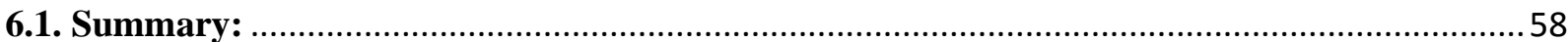

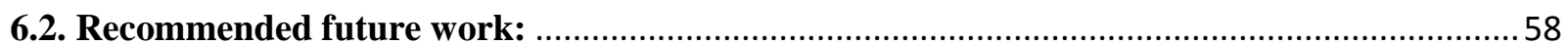

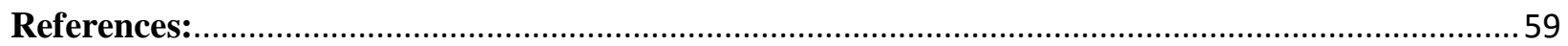




\section{List of Figures}

Figure 1: Schematic representation of various nano structure that can be synthesized using anodization process

Figure 2: Schematic representation of electrospinning system (a) Vertical set-up (b) Horizontal

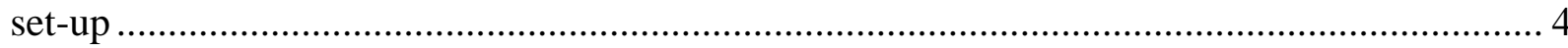

Figure 3: Schematic representation of titanium di oxide nanoparticle coated with relevant drug

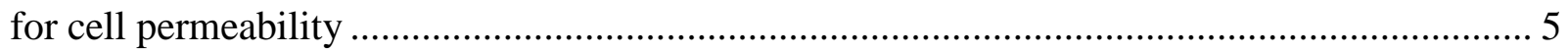

Figure 4: Primary osteoblast cell interacting with various nanomorphologies ....................... 5

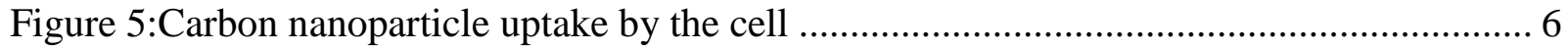

Figure 6: Schematic representation of ultrafast pulsed laser set up ................................... 8

Figure 7: Over all graphical abstract for synthesizing multi-Ti oxide phased nanostructure and its ability to modulate HeLa cell cytoskeleton behaviour ............................................. 13

Figure 8: Graphical illustration of two dominant phases formed in a multi-Ti oxide phased nanostructure by varying the ionization energy of the ultrashort pulsed laser ...................... 16

Figure 9: XRD analysis of multi-Ti oxide phase of nanostructure and its quantitative analysis:

(a) FESEM micrograph of multi Ti-oxide phase titanium nanostructure, (b-c) Apparent core shell morphology of rutile dominant nanostructure,(d-e) TEM-EDX results of anatase dominant and rutile dominant nanostructure, $(\mathrm{f}-\mathrm{j})$ HRTEM images of rutile dominance nanostructure showing the different lattice spacing....

Figure 10: (A) XRD analysis of multi-Ti oxide phase nanostructres ,(B) Quantitative analysis of various phases present in the nanostructures ......................................................... 18

Figure 11: HeLa cell and NIH3T3 cell adhesion on anatase dominance and rutile dominance nanostructure at 24 hours and 48 hours

Figure 12: HeLa cell and NIH3T3 cell on anatase dominance and rutile dominance nanostructure at 24 hours and 48 hours

Figure 13: (1) Anatase dominant nanostructure controlling HeLa cell cytoskeletonat at 24 hours and 48 hours, (2)Rutile dominant nanostructure controlling HeLa cell cytoskeleton

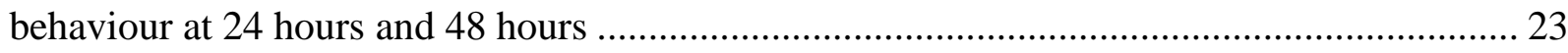

Figure 14: Fluorescent micrographs of HeLa cells on rutile dominant nanostructure and control titanium surface. Scale bar is $10 \mu \mathrm{m}$.

Figure 15: AFM method to analyze the height and phase contrast image of the HeLa cancer cell interacting with rutile dominant nanostructure and the control sample

Figure 16: Surface area of HeLa cell on anatase dominance nanostructure, rutile dominance nanostructure and control titanium sample in 24 hours, units in $\mu \mathrm{m}^{2}$

Figure 17: Graphical abstract illustrating ying-yang proliferative zones using ultrashort pulsed laser 30 
Figure 18: Multiphase titanium oxide nanostructure synthesized by varying the ionization energy of ultrashort pulsed laser, $(a, b, c)$-nanostructure generated by high ionization energy from ultrashort pulsed laser, $(\mathrm{d}, \mathrm{e}, \mathrm{f})$ - nanostructure generated by low ionization energy from ultrashort pulsed laser.

Figure 19: AFM method to analyze the change in height and material composition of nanostructure synthesized by varying ionization energy, $(\mathrm{a}-\mathrm{b})$ height and phase- high ionization energy, (c-d) height and phase-low ionization energy 32

Figure 20: Nanoparticle size determined by varying the ionization energy from the ultrashort pulsed laser, (a) Nanoparticle size varied at high ionization energy, (b) Nanoparticle size varied at low ionization energy .

Figure 21: XRD plot of multiphase titanium oxide nanostructure synthesized by varying the ionization energy, (a) Lattice fringes of multiphase titanium oxide nanostructure generated by high ionization energy, (b) Lattice fringes of multiphase titanium oxide nanostructure generated by low ionization energy ....

Figure 22: HeLa cell count on proliferative zone and non-proliferative zone of the nanomaterial by varying the energy from the ultrashort pulsed laser. 35

Figure 23: HeLa cell count on proliferative zone and non-proliferative zone synthesized by high and low ionization energy from ultrashort pulsed laser 36

Figure 24: Influence of proliferative and non-proliferative zone of the multi-Ti oxide phased nanostructure determine the HeLa cell cytoskeleton shape $37-38$

Figure 25: Influence of various nanopatterns synthesized by high energy from the ultrashort pulsed laser.

Figure 26: Graphical illustration representing the dominant phases of titanium oxide influence HeLa cell - cell signalling. 43

Figure 27: HeLa cancer cell-cell communication influenced by dominant phase of multi-Ti oxide phased nanostructure

Figure 28: Displaying the influence of dominant phases of multi-Ti oxide nanostructure in determining HeLa cell elongation at 24 hours

Figure 29: Influence of dominant phases of multi-Ti oxide nanostructure in multi cell channelling and single cell channelling

Figure 30: Cancer cell directionality is determined by the dominant phase of multi-Ti oxide nanostructure

Figure 31: Influence of dominant phase of multi-Ti oxide phased nanostructure determines the HeLa cell alignment at 24 hours $51-52$

Figure 32: Scanning electron micrograph showing the influence of titanium nano material when interacting with HeLa on (A -C) Anatase dominant titanium nano material (D-F) Rutile and non-stoichiometric cubic $\mathrm{TiO}$ oxide dominant 


\section{List of Abbreviations}

AFM Atomic force microscopy

BSA Bovine serum albumin

CVD Chemical vapor deposition

DMEM Dulbecco’s Modified Eagle Medium

DMSO Dimethyl sulfoxide

DPBS Dulbecco's Phosphate-Buffered Saline

ECM Extra cellular matrix

EDS Energy dispersive X-ray spectroscopy

fs Femtosecond

HAZ Heat affected zone

HEPS 4-(2-hydroxyethyl)-1-piperazineethanesulfonic acid

MEMS Microelectromechanical systems

MHz Megahertz pulse

MTT 3-(4,5-dimethylthiazol-2-Y1)-2,5-diphenyltetrazolium bromide

nm Nanometer

SEM Scanning electron microscope

TEM Transmission electron microscopy

XRD X-Ray diffraction

$\boldsymbol{\mu m}$ Micrometer 


\section{List of Nomenclature}

Apoptosis

Biocompatible

Cytoskeleton

Ex Vivo

Extra Cellular Matrix

Fibroblasts

Filopodia

Focal adhesion

In vitro

In vivo

Integrin

Lamellipodia

Osteoblasts

Proliferation
Death of a cell

Characteristic of not being harmful to tissue

A network of protein filaments that give the shape to a cell

Condition where tests are performed on living tissue outside the body with minimal changes to the natural environment

A collection of molecules that are secreted by cells that provides structural and biochemical support to the cells

A type of cell that synthesizes extra cellular matrix and collagen and has a critical role in wound healing

Slender cytoplasmic projections that aid in migration

Strongest point of adhesion by the cell to the substrate

Tests that take place outside a living organism

Tests that take place inside a living organism

Receptors that form bridges between cell-cell and cell-substrate interactions

Flattened protrusion of the cell that adheres to the substrate

Cells that contribute to bone production

Rapid increase in numbers 


\section{Chapter -1 \\ Introduction}

\subsection{Introduction:}

Nano titanium oxide structures is now recently has the application in cancer therapy because of its excellent photocatalytic, photo excitation capability in destroying cancer cells. There is also application of nanoparticles in genetic engineering where cells can be manipulated depending on the interaction with mammalian cells surrounding them. Cell imaging and as a carrier for drug delivery is the application where titanium oxide nanoparticles was proven candidate. Metallic nanostructures is a synthetic material, which replace a part or function of the human body using implants or to function in intimate contact with living tissue in a safe, reliable, economic and physiologically acceptable manner in a human body. Up to date plenitude of research work has unveiled the role of nanostructures in harmonizing the response of cells for delicate control over their adhesion and proliferation by engineering the biomaterials. Currently regulation of the cancer cells was achieved through properties including surface chemistry, topology, varying the spaces in-between the nanostructures 3. However there were greater efforts taken in killing cancer cells by doping nitrogen with titanium oxide nanostructures because ROS generation where species such as $\mathrm{O}_{2-}, \mathrm{H}_{2} \mathrm{O}_{2}, \mathrm{OH}^{-}$where by applying photodynamic therapy.

Current cancer therapy approach is through chemotherapy and radiation treatment whose treatment is the most effective. However, these approaches would completely damage the cancer cells but also surrounding healthy mammalian cells. These methods have a non-specific mode of action but further anti-cancer effects is induced by chemotherapeutic agents such as paclitaxel and doxorubicin. Also these methods are whole body treatments which results in side effects where deterioration in the quality of life.

Nanostructures cues play the role of extracellular matrix in modulating the cellular behaviour such as adhesion, migration, proliferation and differentiation. The relationship between the cells and the ECM is complex and suggest great attention is required to control the charteristics of the cell. Many research studies have been analyzed to understand the fibroblast ${ }^{4}$, epithelial cells ${ }^{5}$, neuron

cells ${ }^{6}$ behaviour but not sufficient research work on controlling the cytoskeletal behaviour of cancer cells. However membrane bound heterodimeric receptor - Integrin acts as a communicator 
in-between the ECM and the cells intercellular membrane act with. Biological molecules are in inactive state and they freely interact with the available cellular membrane to form a binding domain with the nanostructures. For practical applications the major requirement for developing the engineered nanofabrication approach is to allow processing the materials with desired structural, mechanical, optical, magnetic and electrical properties. The nanofabrication technique is divided into two major categories in creating nanostructures: top-down and bottom-up approaches. In top-down approaches, the desired shapes and characteristics of the nanoscale structures can be synthesized from larger dimensioned features. While in the bottom-up approaches, molecular components are built up into complex nanoscale structured assemblies ${ }^{6,7}$.

\subsubsection{Titanium as a biomaterial:}

Titanium is a common and well-established biomaterial that is widely used in dental, orthopedic and cardiovascular implants because of its biocompatibility, biodegradability, strong chemical stability and mechanical strength ${ }^{8}$. Also titanium is now being proposed to be used as a carrier for drugs and to reduce magnetic resonance imaging artifacts ${ }^{9}$. When titanium is exposed to the atmosphere, protective $\mathrm{TiO}_{2}$ stable oxide film is formed that provides the ability to absorb proteins due to high surface to volume ratio thereby inducing a platform for cells to differentiate. Findings imply that approaches to modulate cell migration behaviour include varying nanotopography of the substrate ${ }^{10-12}$, controlling spacing in between the nanostructures and drug loading of nanotubes ${ }^{13,14}$. These approaches have provided numerous intrinsic investigations and understanding the factors governing cell adhesion onto nanostructure are limited to specific morphology such as nanotubes, nanorods and nanowire. There are no reported studies where the nanostructures' size has remained the same but the material composition of the nanostructure is varied for studying different effects responsible for cancer cell behaviour ${ }^{12}$. In particular, designing an effective nanostructure that governs HeLa cell cytoskeletons but allows mammalian cell to spread has remained a fundamental challenge ${ }^{8}$.

\subsubsection{Methods of Fabricating Titanium Oxide Nanostructures:}

The most common methods nanostructures synthesized for manipulated cell is anodization process, electrospinning process and nanoparticle generated through ball milling process. However in the anodization process, nanotubes is the most common morphology synthesized. 


\subsubsection{Anodization process:}

This multistep process involves pre-treatment of titanium material is subjected to several voltages for a period of time which leads to the formation of nanotube structure over the surface. Then the nanotube structure is removed by ultrasonically in deionized water and by exposing the glossy underlying surface at the substrate. Further synthesizing of the nanotube structure is achieved by using the glossy surface by applying the electrical voltage. There are attractive methods to synthesize the nanotubes by controlling the fluoride presence in an electrolyte resulting in highly ordered layers of nanotube ${ }^{15,16}$. Precise control of the nanotube diameter is achieved in phosphate fluoride electrolyte presence by applying the voltage from $1 \mathrm{~V}$ to $20 \mathrm{~V}^{17}$. Length of the nanotube is controlled by the duration of the anodization process however the final length of the nanotube is dependent on the voltage applied and the type of electrolyte Stimulated cell response is achieved when the spacing of the nanotubes is achieved at $15-30 \mathrm{~nm}^{10}$. There are few research where they have evidence that in the presence of aqueous electrolytes the nanotubes are not in uniform shape and replies are formed outside of the tube walls. These ripples make the nanotube structure mechanically unstable which leads to distortion and cells won't be able to adhere ${ }^{15}$.

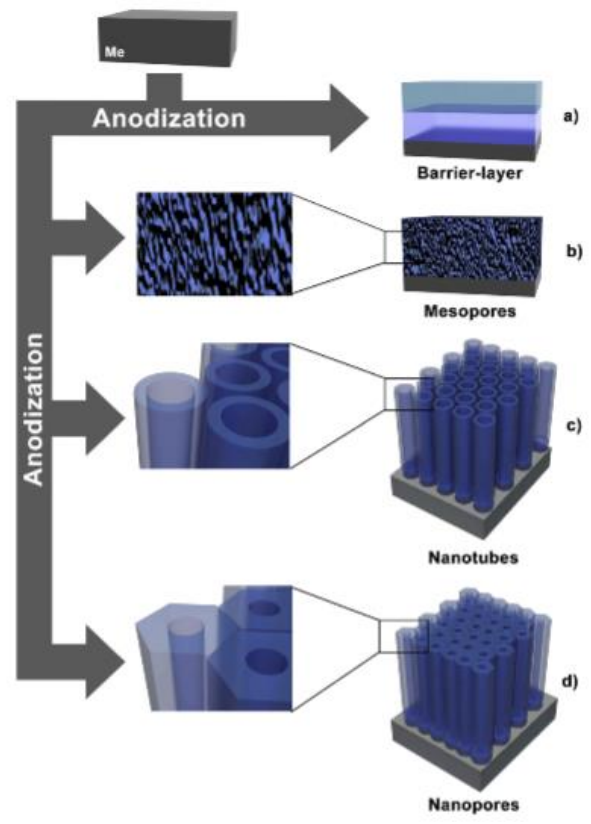

Figure 1: Schematic representation of various nano structure that can be synthesized using anodization process 


\subsubsection{Electrospinning technique:}

Electrospinning is the process which utilizes the polymeric solution driven from a syringe into needle by a syringe pump to synthesize polymeric fibers ranging from $2 \mathrm{~nm}$ to several micrometers ${ }^{18,19}$. It is the only process where controllable pore structure can be achieved however the application of the smaller pore size, high surface area, malleability to conform to a wide variety of shape and size has gained attention in the field of nanocatalysis, tissue engineering scaffold, protective clothing, filtration, biomedical and environmental engineering ${ }^{20}$. There has been increasing interest in fabricating nanofibrous scaffold using natural and synthetic fibers such as polylactic acid ${ }^{21}$, polyurethanes ${ }^{22}$, silk fibroin ${ }^{23}$, collagen $^{24}$, hyaluronic acid $^{25}$, cellulose ${ }^{26}$, chitosan and collagen ${ }^{27}$.Despite several advantages because of their versatility for wider application, there is a bottle neck problem where single jet electrospinning system cannot synthesize larger quantities. However the major challenge in using the electrospun nanofibers in tissue engineering is nano-uniform cellular distribution and the lacking of cellular migration which is an important application for wound healing ${ }^{28}$.

a)

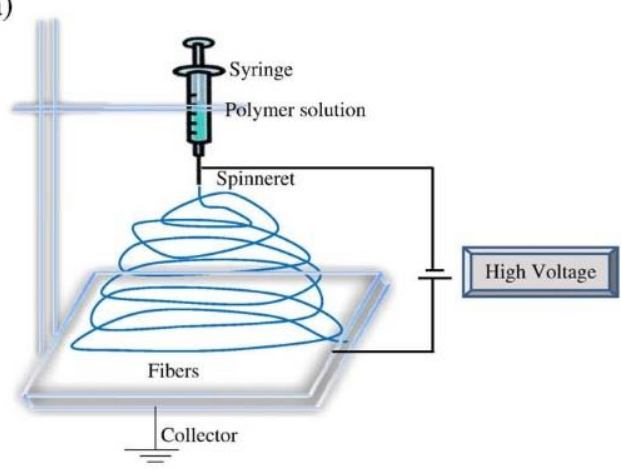

b)

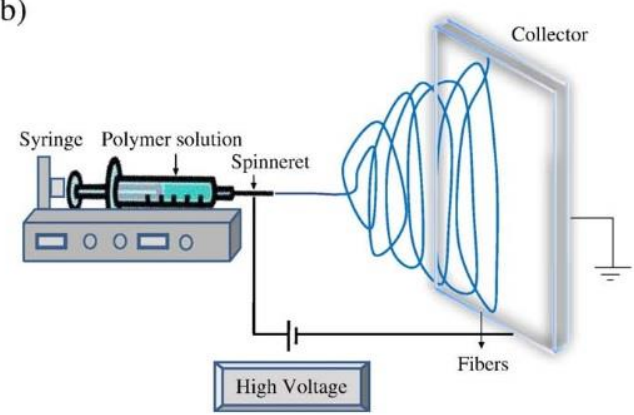

Figure 2: Schematic representation of electrospinning system (a) Vertical set-up (b) Horizontal set-up 29 


\subsubsection{Titanium nanostructure as a drug carrier:}

Titanium nanoparticle widely used because of their unique physio-chemical characteristics having wide application such as sunscreen, cosmetic, medicine, paints, textiles, plastics, paper, industrial products and photocatalytic process ${ }^{30-33}$. However because of their unique size, they have an important application in medical industry where they can be used as drug delivery systems, targeted cancer therapy, bio sensing application, and nano-imaging. Recently $\mathrm{TiO}_{2}$, which is earlier used in semiconductor industry was now used as a photosensitizer in photodynamic therapy because of their excellent biocompatibility, photo reactivity and low toxicity. But these materials when react with water results in releasing reactive oxygen species which is harmful to cells. This interaction with the cells would not be selective targeting of cancer cells ${ }^{34}$. The limitations of this method is only titanium-di-oxide nanostructure can be used as drug carrier and also drugs loaded into nanotubes has the ability to withhold only limited capacity of drug.

B

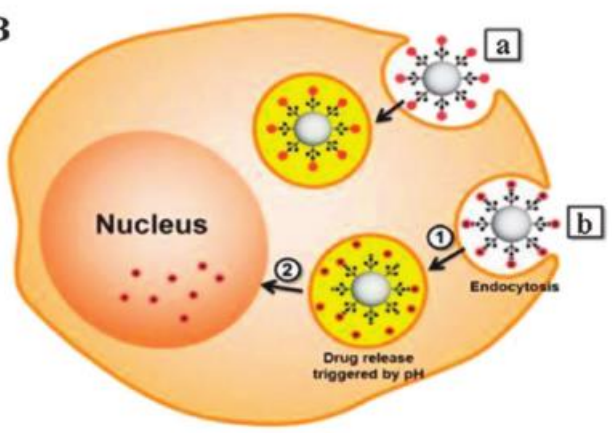

A

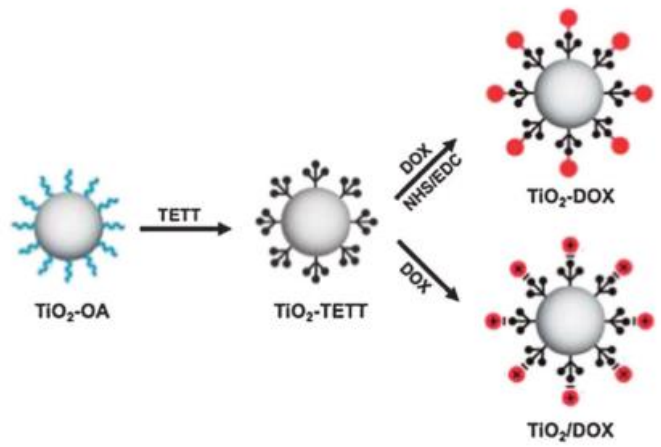

Figure 3: Schematic representation of titanium di oxide nanoparticle coated with relevant drug for cell permeability ${ }^{35}$ 


\subsection{Influence of various morphology of nanostructures for targeted therapy:}

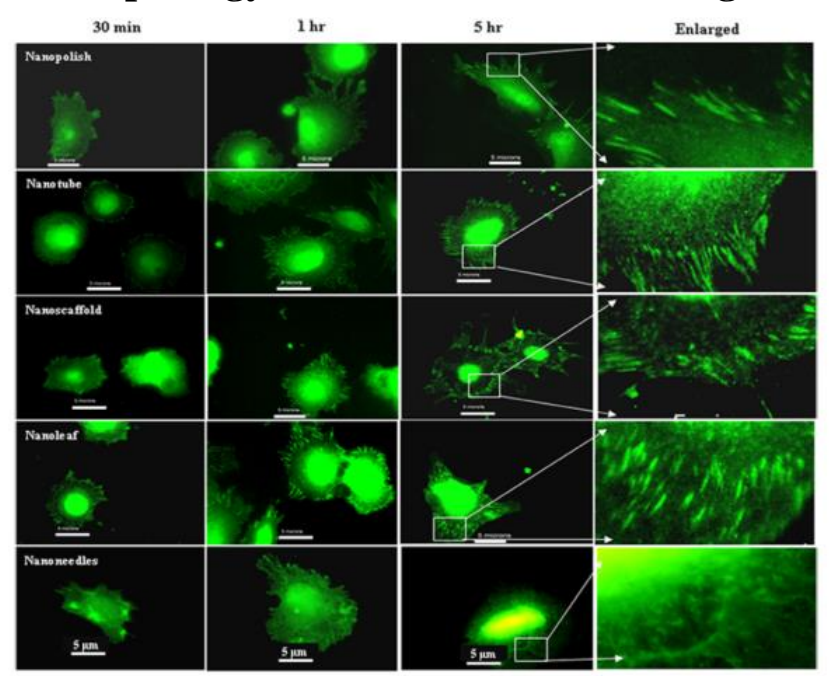

Figure 4: Primary osteoblast cell interacting with various nanomorphologies ${ }^{33}$

In the figure 4, it is observed how osteoblast cells interaction with nanotube, nanoscaffold, nano leaf and nanoneedles where enlarged actin stress fiber are formed when compared to nano needles. This indicate that needle shaped morphology of the nanostructure does not allow adhesion of cells 33. However there is evidence of material composition of the nanostructure determines the cell adhesion and proliferation. Limitation of this method is single phases of $\mathrm{TiO}_{2}$ nanostructure is synthesized and used for the application in cell manipulation. Also different morphology of the nanostructure has influence in directing cell polarization and migration.

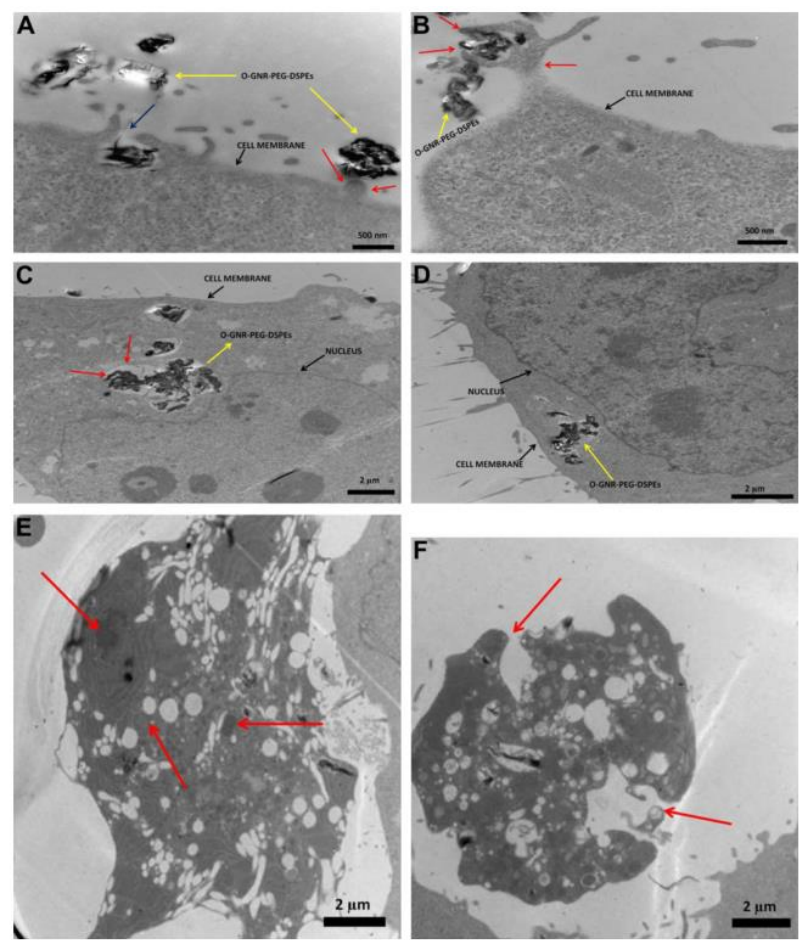


Figure 5: Carbon nanoparticle uptake by the cell ${ }^{36}$

In the figure 5, oxidized graphene nanoribbons and amphiphilic polymer enclosed nanoribbons are interacted with the HeLa cervical cancer cell. Nanoparticle uptake by the cell is clearly observed through TEM cross section. This indicate that the cell uptake of the nanoribbons results in necrosis where outer cell membrane of the cell is penetrated 36. Depending on the cytotoxicity of the material, cell death happens. However there are few research where nanoparticle is coated will cell specific material which enables them to penetrate.

\subsection{Summary:}

Nanostructures is the only method that can intervene cancer cells at molecular level however this is achieved in using them as targeted therapy and as a drug carrier. However these methods resulted in manipulating cell by varying the individual nanostructure morphology for directing cell polarization, varying the distance in-between the nanostructures in preventing focal adhesion of the cell adhering. Also these nanostructures are made up off uniform material composition whereby resulted in specific application of allowing cell proliferation only. Therefore these results in quested us to wonder whether having a nanostructure can be made up off multiple phases of various combination of titanium oxides. The gap in the current research was to synthesize phase dominance in multi-Ti oxide nanostructure for the application of regulating cancer cell cytoskeleton behavior. In this dissertation, dominant phase of multi-Ti oxide nanostructure having 3-D randomly oriented nanostructure was synthesized for manipulating selectively HeLa cancer cell not the mammalian cells.

\subsection{Research objective:}

The ultrashort pulsed laser interaction with the titanium substrate has an important characteristics of controlling HeLa cancer cell proliferative behaviour and manipulating their cytoskeleton behaviour using multi-Ti oxide nanostructure. This distinct material fabrication technique enables us to tune unique composition of titanium oxide phases for the application of targeted cancer cell therapy. The main objective of this thesis is to synthesize three dimensional nanostructures having 
multiples phases of titanium oxides using ultrafast pulsed laser. The three sub-objective that is accomplished through the following tasks:

Sub Objective 1 Create dominant phases of titanium oxides in a nanostructure

Task 1 Harmonizing HeLa cell cytoskeleton behavior by multi-Ti oxide nanostructure

Sub Objective 2 Create ying-yang proliferative nanostructure zones having dominant phases of titanium oxides

Task 2 Study HeLa cell patterning using ying-yang proliferative nanostructure zone

Sub Objective 3 Cancer cell signalling influenced by dominant phases of multi-Ti oxide phases in a nanostructure

\subsection{Organization of thesis:}

Chapter -3

Harmonizing HeLa cell cytoskeleton behaviour by multi-Ti oxide phased nano structure synthesized through ultrashort pulsed laser

$>$ FESEM, TEM, EDX: Study the morphology of multi-Ti oxide phased nanostructure

$>$ XRD: Quantitative and qualitative analysis of phases of titanium oxide

$>$ AFM: Qualitative analysis of nanostructure with HeLa cell

Chapter -4

HeLa cell patterning by Ying-Yang proliferative zones using multiphase titanium oxide nanostructure synthesized by ultrashort pulsed laser

$>$ Fluorescence: Study the actin cytoskeleton and nuclei of cells in the proliferative and nonproliferative zone

Chapter -5

Cancer cell-cell signalling under the influenced by dominant phases of multi-Ti oxide nanostructures

Fluorescence: Alignment and elongation of HeLa cells on multi-Ti oxide phased nanostructure

\subsection{Publication:}

1. Manuscript under review "Harmonizing HeLa cell cytoskeleton behaviour by multi-Ti oxide phased nano structure synthesized through ultrashort pulsed laser "in Nature Scientific reports, 2015 , Impact Factor 5.578 


\section{Chapter 2}

\subsection{Materials and methods:}

\subsubsection{Synthesizing multi-Ti oxide phased nanostructure formation throughultrafast}

\section{femtosecond pulsed laser:}

Simple but effective tuning of multi-Ti oxide phased nanostructures with pattern regularity is achieved through ultrashort pulsed laser interaction. Titanium sample (ASTM B265) grade were ground finished by grit silicate carbon paper to remove macro level defects and finally machine polished using alumina. The irradiation from ultrashort pulsed laser source was constituted by 1040nm wavelength direct-diode-pumped Yb-doped fiber amplified femtosecond laser system (Clark MXR) with an average power of $16 \mathrm{~W}$ and repetition rate ranging from $4 \mathrm{MHz}$ to 26MHZ.The titanium sample was mounted on a precision X-Y-Z stage normal to the ultrashort pulsed laser beam.

Ultrashort pulsed laser interaction technique is an unique non-contact nanostructure processing approach that can facilitate in synthesizing various combination of multi-Ti oxide phases in a nano structures through the vapor condensation mechanism where the material undergoes a phase transformation through melting and vaporization as well as phase explosion if laser fluence are sufficiently high.

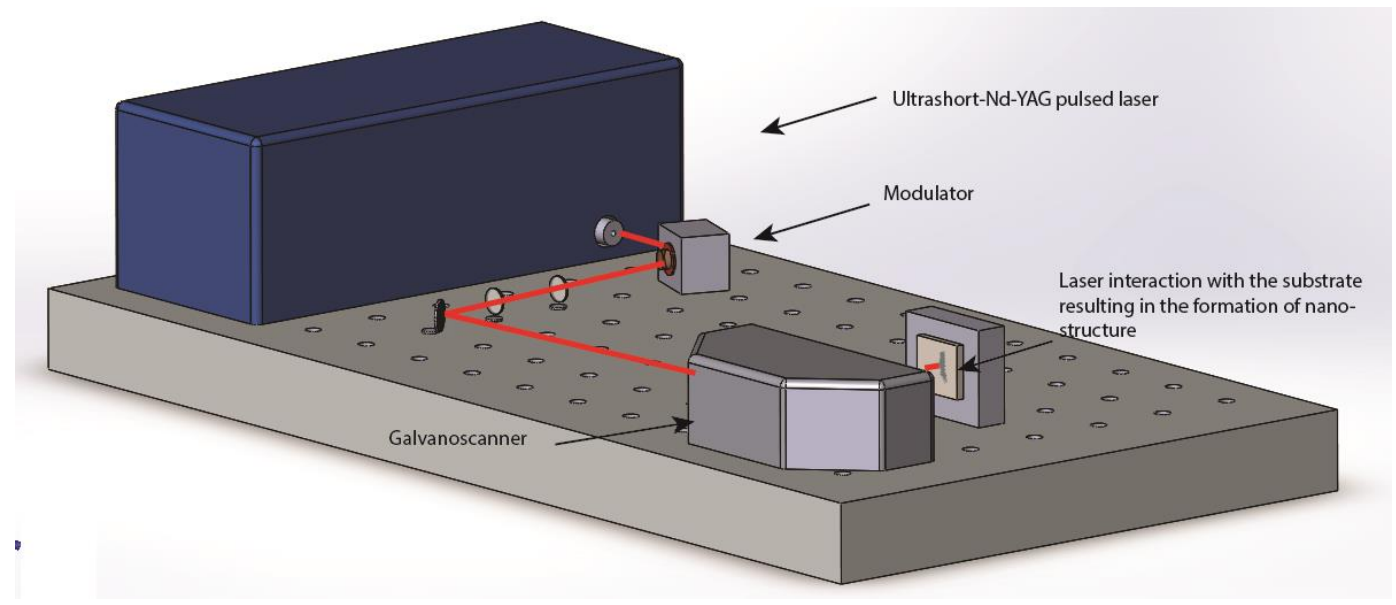

Figure 6: Schematic representation of ultrafast pulsed laser set up

\subsubsection{Cell culture:}

HeLa, human cervical cancer cell line was obtained from ATCC (American type culture collection, ATCC No. CCL-2) and were cultured in DMEM-F12 medium with Figure 1 Schematic 
representation of ultrafast pulsed laser set-up phenol red containing $10 \%$ heat inactivated fetal bovine serum and $1 \%$ penicillin-streptomycin antibiotics at $35^{\circ} \mathrm{C}$ in $5 \% \mathrm{CO}_{2}$. NIH3T3, fibroblast cells was grown in DMEM medium containing $10 \%$ heat activated fetal bovine serum with $1 \%$ penicillin-streptomycin antibiotics at $35^{\circ} \mathrm{C}$ in $5 \% \mathrm{CO}_{2}$.

\subsubsection{Scanning electron microscopy:}

The specimen multi - Ti oxide phase nano structure was qualitatively evaluated using field emission-scanning electron microscope (Hitachi, SU-8200) and HeLa cell seeded onto the sample were analyzed by using scanning electron microscope (Hitachi, SU-1500). To study the interaction of cell with the nanostructure they were incubated at 24 hours and 48 hours. After incubation the spent medium was removed and the sample is fixed by glutaraldehyde for 30 minutes at $4^{\circ} \mathrm{C}$. Subsequently sample was washed twice with $1 \%$ sodium cacodylate buffer at $4^{\circ} \mathrm{C}$. After that cell were dehydrated through a graded ethanol series (from $10 \%$ to $100 \%$ ) for $15 \mathrm{~min}$. Then samples are critical point dried and prior to SEM examination, samples were sputtered with gold layer.

\subsubsection{X-ray diffraction:}

The elemental composition data of multi-Ti oxide phase nanostructure was collected on Bruker AXS D8 Advance micro diffraction system and it is equipped with $\mathrm{Cu}-\mathrm{Ka}$ source and a graphite monochromatic for elimination of unwanted $\mathrm{Cu}-\mathrm{K}$-beta lines. The interaction of the incident rays with the sample creates an interference and the diffracted X-rays is detected, processed and scanned through 2D detector. All the possible lattice planes were obtained by calculating the dspacing, thus allows us to identify the individual mineral. Each mineral has a signature d-spacing, which is compared with the standard patterns for identifying the phase corresponding to diffraction peaks.

\subsubsection{Fluorescent staining of cells:}

The samples are first fixed in paraformaldehyde followed by incubation in milk to prevent nonspecific binding. To stain the actin and cytoskeleton, the samples are incubated with Alexa fluor 488(Life Technologies) followed by DAPI (4', 6'-diamidino-2-phenylindole, Life Technologies) to stain the nucleus. An epi-fluorescent nikon E-400 microscope was used and data were recorded by DS-5M-U1 color digital camera (Nikon, Canada). 


\subsubsection{Measuring cellular alignment using $\mathrm{F}$-actin:}

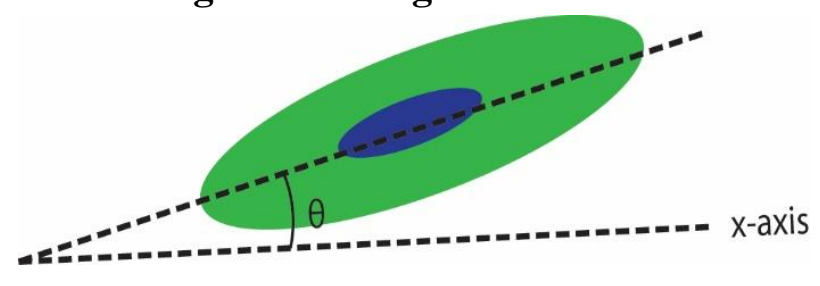

Illustration represents the angles that were measured within the cell in order to carry out fiber alignment angle measurements. Cell outline is shown in green and nucleus in blue. Angle $(\theta)$ is the angle that is formed between the $\mathrm{x}$-axis and the long axis of the cell.

\subsubsection{Nanoparticle size measurement:}

This analysis was performed using HR-TEM to obtain the nanoparticle to measure its shape and size. HR-TEM measurement involves the preparation using carbon grid to swab the sample the nanoparticles to the grid and then grid is scanned. Also using lattice fringes of the nanoparticle, crystal orientation was determined.

\subsubsection{AFM analysis:}

AFM is used to measure the phase contrast and topographical contour mapping of the titanium nanomaterial and their interaction with HeLa cell. They were mapped in the non-contact mode using NT-MDT, AFM, Russia equipped with prefabricated cantilever at scan rate of 30 min and all images were acquired at a scale of 100 X $100 \mu \mathrm{m}$ for better resolution. All the images obtained by AFM are measured using the same cantilever and identical scanning conditions.

\subsubsection{Statistics:}

All experiments were triplicated and data represented as mean \pm standard deviation unless otherwise mentioned. 


\section{Chapter -3 \\ Harmonizing HeLa cell cytoskeleton behavior by multi-Ti oxide phased nano structure synthesized through ultrashort pulsed laser}

Multi-Ti oxide nanostructures with controllable dominant phases in titanium oxides were synthesized by ultrashort pulsed laser interaction with the $\alpha$-phase titanium. In this way, by varying the ionization energy from the ultrashort pulsed laser either anatase dominant phase in multi-Ti oxide nanostructure or rutile dominant phase in multi-Ti oxide nanostructure. Such unique combination of titanium oxide phases served as a proliferative behaviour for mammalian cells but not HeLa cancer cell indicates that nanostructured material can be effectively designed for targeted cancer therapy. There was change in cytoskeleton behavior including the various cytoskeleton shape, reduction in surface area of the cell.

\subsection{Introduction:}

Regulating cancer cell behaviour is a complex biological process, where there is a need to restrain the cytoskeletal arrangement by bio-mimetic nano structured materials ${ }^{12,39,40}$. This communication is mediated by the direct interaction between cell surface receptors and physical extra cellular matrix (ECM) molecules. Mnemonic ability of these ECM's plays an imperative role in regulating cancer cell behaviour such as cell adhesion, spreading, proliferation, differentiation, gene expression and signal transduction ${ }^{41-43}$. Two approaches that synthesize the physical ECM are biocompatible surface nanostructure topography and composing hydrophilic functional groups on nanostructures to initiate specific cellular response.

The dialogue between surface nanostructure materials and the cancer cell behaviour is a result of external biophysical stimulus, which is crucial for understanding many fundamental biological questions in designing biomaterial ${ }^{44}$. Anodization is one of the primary techniques that can synthesize the surface nanostructure topography ${ }^{45}$. A majority of experimental evidence indicates that the nanotubes increases focal adhesion formation and subsequent cell spreading ${ }^{46}$. However in the anodization process there is no control over construction of nanotubes with uniform material composition, and due to the extended anodization process the outermost tubes becomes 
substantially thinner and disintegrate ${ }^{47}$. As a result nanotube feature size and material composition is of great concern in regulating cancer cell behaviour.

Functionalizing the titanium nanotubes by combining them with drug delivery systems including functional proteins ${ }^{48}$, growth factor enclosed in fibrin hydrogels ${ }^{49}$ and anti-inflammatory drugeluting compounds ${ }^{50}$ determines the cell fate : cell adhesion, proliferation and differentiation. Commonly used methods for surface coating the titanium nanotubes are Sol-gel, evaporationinduced surface crystallization and spin assisted layer-by-layer technique ${ }^{51-53}$. For instance Mehdi Kazemzadeh-Narbat et al., reported that phospholipid coating of $\mathrm{CaP}$ in titania nanotube shows a reduction in bacterial growth but allows proliferation of bone cell. However the limitations on loading capacity of the drug onto the nanotubes is dependent on the structural parameters such as tube diameter and length ${ }^{54}$. In order for cells to interact with nanotubes their optimum diameter is $15 \mathrm{~nm}$, which indicates the limitations in releasing drug for longer duration ${ }^{10,55}$.

Titanium is a common and well-established biomaterial that is widely used in dental, orthopedic and cardiovascular implants because of its biocompatibility, biodegradability, strong chemical stability and mechanical strength ${ }^{8}$. Also titanium is now being proposed to be used as a carrier for drugs and to reduce magnetic resonance imaging artifacts ${ }^{9}$. When titanium is exposed to the atmosphere, protective $\mathrm{TiO}_{2}$ stable oxide film is formed that provides the ability to absorb proteins due to high surface to volume ratio thereby inducing a platform for cells to differentiate. Findings imply that approaches to modulate cell migration behaviour include varying nanotopography of the substrate ${ }^{10-12}$, controlling spacing in between the nanostructures and drug loading of nanotubes ${ }^{13,14}$. These approaches have provided numerous intrinsic investigations and understanding the factors governing cell adhesion onto nanostructure are limited to specific morphology such as nanotubes, nanorods and nanowire. There are no reported studies where the nanostructures' size has remained the same but the material composition of the nanostructure is varied for studying different effects responsible for cancer cell behaviour ${ }^{12}$. In particular, designing an effective nano-structure that governs HeLa cell cytoskeletons but allows mammalian cell to spread has remained a fundamental challenge ${ }^{8}$.

The objective of the current research work is the ability to generate the combinatorial titanium oxide phased nanostructure that selectively allows fibroblast cells to proliferate but control the 
differentiation of HeLa cancer cells. Ultrashort laser pulses allows the formation of various combinations of multi-Ti oxide phases in a nanostructure which cannot be achieved through any other fabrication method. Ionization energy of the laser pulses and pulse to pulse separation time were able to induce various concentrations of multi-Ti oxide phases in the nanostructure. Results indicate that at high ionization energy at shortest pulse to pulse separation time displayed a material chemistry property of rutile phase dominance along with the presence of hongquiite phase in this multi-Ti oxide phased nanostructure. Rutile phase dominant with the presence of hongquiite phase nanostructures resulted in non-proliferation of the HeLa cancer cell but simultaneously allowed proliferation of fibroblast cell. Thus the consequence of such generation of multi-Ti oxide phase nanostructure has opened up the possibility to judiciously alter the entire material chemistry of these multi-Ti oxide phased nanostructure while at the same time retaining the continuous formation of nano structure.
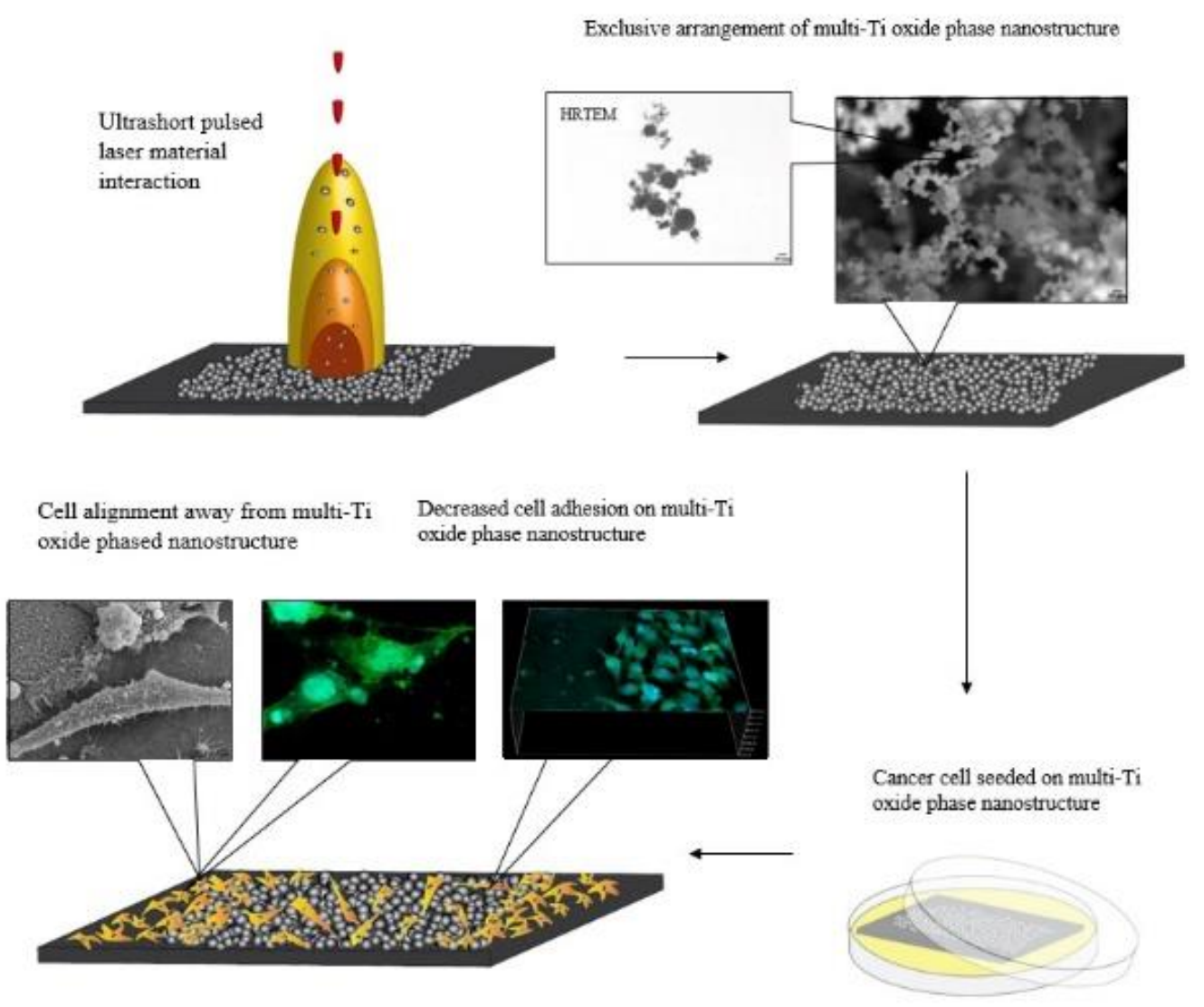

Figure 7: Over all graphical abstract for synthesizing multi-Ti oxide phased nanostructure and its ability to modulate HeLa cell cytoskeleton behaviour 


\subsection{Results and Discussions:}

\subsubsection{Synthesizing multi-Ti oxide phased nanostructures using ultrashort pulsed laser:}

Laser ablation plume is a highly dynamic and non-linear process. The interaction of plume in the presence of ambient gas is a highly elaborate gas dynamic process involving various steps: surface adsorption of laser, vaporization , plasma ignition, plasma adsorption and rapid cooling followed by condensation ${ }^{56}$. When the energy delivered by the ultrashort pulsed laser is in excess to the binding energy of that atom it breaks by means of repetitive laser pulses. When ultrashort pulsed laser interacts with the target material the surface gets heated up to higher temperature and they combine with air which is present in the background to obtain the energy required for vaporizing the material and thus plasma plume is formed consisting of electrons and ionized atoms. When this plasma plume expands outward there is a heat transfer in-between the plume and the ambient gas, results in cooling down of plume and the condensation process begins. This condensation process results in nucleation step, where the growth of the supercritical nuclei initiated and comes to a halt by quenching. Thus the growth of nanoparticles is initiated they aggregate due to the collision of nuclei and hence 3-D nanostructures are formed. When these ultrashort laser pulses are focused onto the target material only the localized region within the focal volume absorbs the energy by a nonlinear process, such as multiphoton avalanche ionization, resulting in minimizing the thermal stress and collateral damage. The plume diffusion time depends on the various conditions: laser parameters, material properties and the ambient gas condition ${ }^{57}$. Earlier studies revealed that only $\mathrm{MHz}$ laser pulse repetition rates can keep the atomic flow into the plume at the critical level in order to synthesize 3-D nanostructures ${ }^{58,59}$.

Evaporation rate of single pulsed laser can be expressed as

$$
\left\langle R_{\text {evp }}\right\rangle \text { therm } \approx n_{\text {air }}\left(\frac{k_{B} a A t_{\text {eq }}^{3 / 2}}{M_{a} k_{h} \pi^{3 / 2}} \frac{P_{\text {avg }}}{R_{\text {rep }} A_{\text {foc }}}\right)^{1 / 2}\left[\frac{\text { atom }}{c m^{2}}\right]
$$

Number of atoms evaporated from a single spot after multiple laser pulses interact at time interval Dt is expressed as

$$
N_{m p} \approx N_{p} R_{r e p} D_{t}=R_{e v p} A_{f o c} R_{r e p} D_{t}[\text { atoms }]
$$

Therefor by substituting Eq. 1 into Eq.2, the number of atoms evaporated by multiple laser pulses based on laser parameters and the material property is expressed as 


$$
N_{m p} \approx n_{\text {air }}\left(\frac{k_{b} a A t_{e q}^{3 / 2}}{M_{a} k_{h} \pi^{3 / 2}}\right)^{1 / 2}\left(P_{\text {avg }} R_{\text {rep }} A_{\text {foc }}\right)^{1 / 2} D_{t}[\text { atoms }]
$$

Thus the pulse to pulse separation time is directly controlling the evaporation rate of the atoms and similarly the pulse repetition rate influence the number of evaporated atoms by a square root. In order to maintain the quality of these 3-D nanostructures and their overall repeatability, the pulse to pulse separation time is the controlling factor where electron excitation and energy transfer occur in two separate phases. However varying the repetition rate $(\mathrm{MHz})$ of the ultrashort laser pulses results in bringing the temperature of the target surface to the temperature where the synthesized 3-D nanostructures are oxidised depending on the ionization energy induced by this ultrashort laser pulses.

\subsubsection{Mechanism of multi-Ti oxide phased nanostructure formation by ultrashort pulsed laser:}

The mechanism of phase transformation in a material is fundamental for controlling material characteristics of the synthesized nanostructure. Among the oxides formed, $\mathrm{TiO}_{2}$ is known for its polymorphism, and they exist in anatase, rutile and brookite phases. The stability of this polymorph is critical, and the kinetics of the parameters is essential in obtaining multi-Ti oxide phased nanostructures. The phase transformation of anatase to rutile is invariable, and they begin to transform in the temperature range varying from $400-1200^{\circ} \mathrm{C}^{60-63}$. Phase transformation of anatase to rutile is referred to as nucleation, and growth process is dependent on variables such as heat flow condition, temperature and time. Thermodynamic phase stability of $\mathrm{TiO}_{2}$ polymorph is stable in the ascending order anatase, brookite, rutile which indicate that anatase and brookite are in less stable form when comparing with rutile ${ }^{64}$. This study shows that when the ionization energy is of higher magnitude, the titanium nanostructure formed is completely rutile phase dominant with the presence of hongquiite phase. However this hongquiite phase is not present when the ionization energy is of lower magnitude resulting in the formation of anatase dominant titanium nanostructures. This indicates that at high energy and at very short condensation time,inbetweenlaser pulses they results a higher amount of ionization energy resulting in phase transformation of anatase into rutile. Low energy and longer condensation time in-between pulses results in low ionization energy, leading to completely anatase phase dominant nanostructure ${ }^{56}$. Thus there remains a question why anatase in a less stable state is predominantly formed in $\mathrm{TiO}_{2}$, when the ionization energy is lower. Extensive research is needed to develop a clear understanding 
of how ionization energy plays an important role in determining phase transformation in nanostructure. 


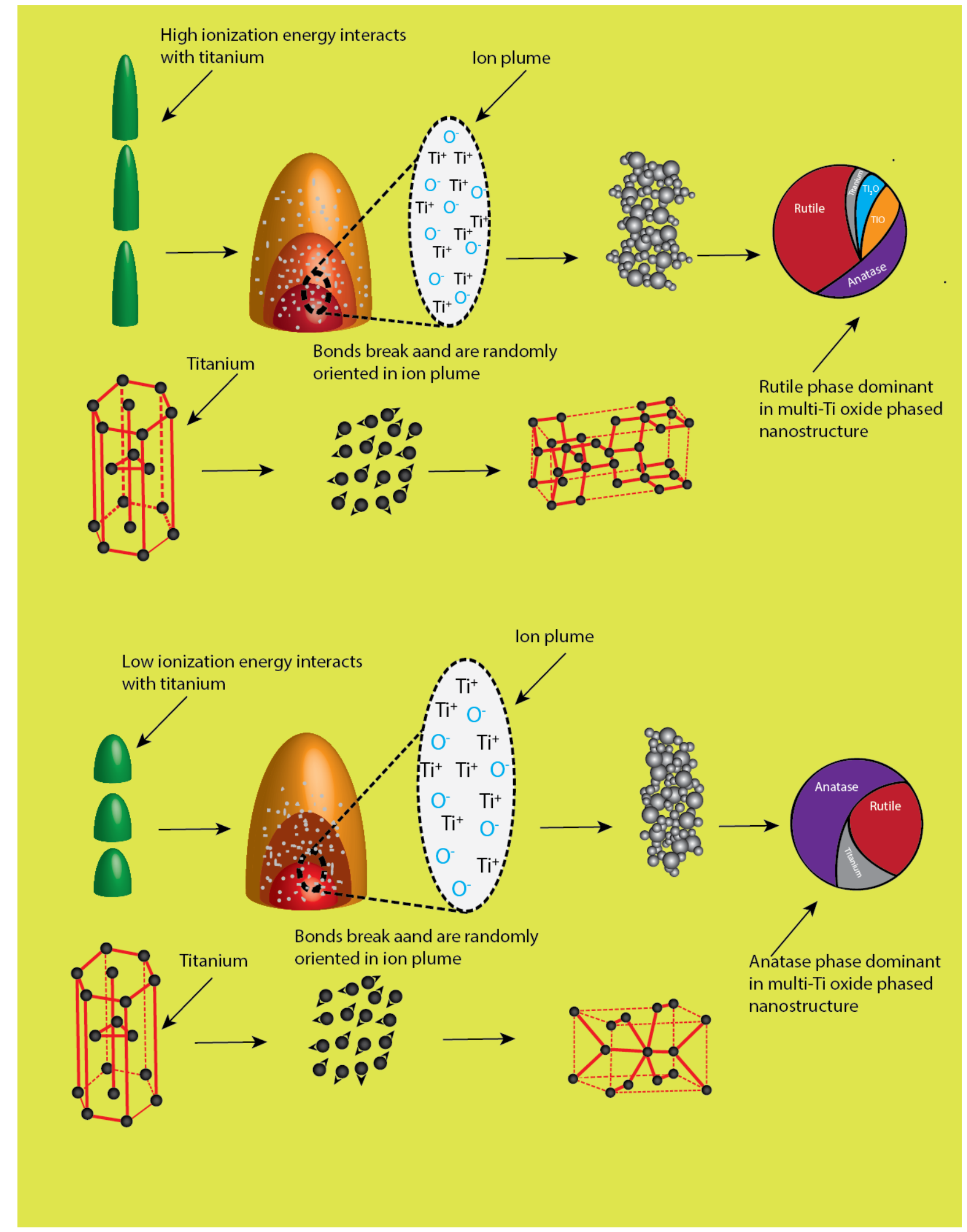

Figure 8: Graphical illustration of two dominant phases formed in a multi-Ti oxide phased nanostructure by varying the ionization energy of the ultrashort pulsed laser 


\subsubsection{FESEM and HR-TEM-EDX analysis of multi-Ti oxide phased nanostructure:}

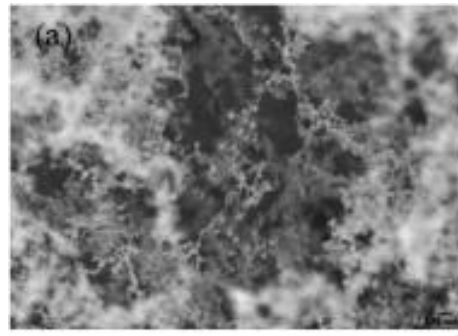

(b)
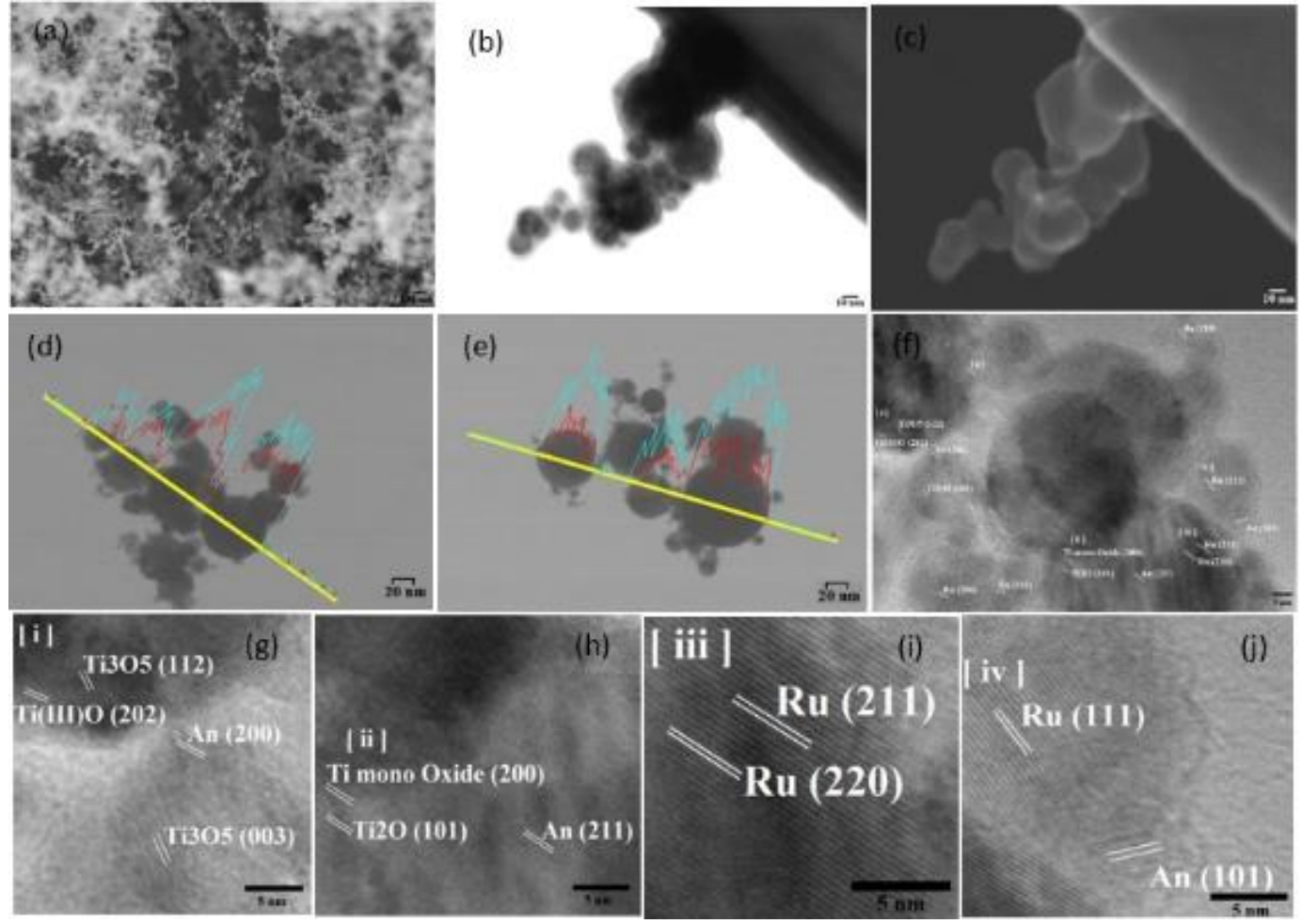

Figure 9: XRD analysis of multi-Ti oxide phase of nanostructure and its quantitative analysis: (a) FESEM micrograph of multi Ti-oxide phase titanium nanostructure ,(b-c) Apparent core shell morphology of rutile dominant nanostructure,(d-e) TEM-EDX results of anatase dominant and rutile dominant nanostructure,(f-j)HRTEM images of rutile dominance nanostructure showing the different lattice spacing

When the ionization energy from the ultrashort pulsed laser interacts with titanium material, this reveals the formation of 3-D multi-Ti oxide phased nanostructure; in this study the arrangement and structural composition of multi-Ti oxide phased nanostructure is analyzed. A close-up view of rutile dominant nanostructure arrangement (figure 3(a)) indicates that it is an agglomeration of self-assembled randomly organized closed rings or chains and they are connected together by a small necking as observed in figure 3(b) and 3(c). Individual nanoparticles are aggregated together and the mere loose packing in-between the nanoparticles is strong because of their bonds ${ }^{56}$. This structural arrangement of the nanostructure remains randomly arranged and display no particular pattern . The back scattered analysis of this rutile dominant nanostructure reveals that the individual nanostructure is made up-of two layers: apparent core-shell morphology. Also this similar 
arrangement is also formed on anatase dominant nanostructure. The TEM-EDX analysis reveals that there is variation in titanium and oxygen peaks in the individual nanostructure when the ionization energy is varied, and thus it is presumed that the outer shell is composed of various titanium oxides, which is validated by XRD analysis. Anatase dominant nanostructure is predominantly formed when the ionization energy is low (figure 3(d)) and the results obtained by TEM-EDX analysis provide the evidence of variation in titanium and oxygen peaks which indicate the there is formation of various phases of titanium oxides. However when compared to anatase dominant nanostructure there is a significant variation in titanium and oxygen peaks of rutile dominance nanostructure (figure 3(e)) that is formed due to higher ionization energy. For further verification, distance between the lattice fringes was measured and identified for rutile dominant nanostructure which indicate there is diverse planes of anatase, rutile , titanium oxide $\left(\mathrm{Ti}_{3} \mathrm{O}_{5}\right)$, titanium mono oxide formed at when high ionization energy interacts with titanium material. This analysis confirms the mixed-oxide phases of titanium formed which is further analyzed by the XRD analysis.

\subsubsection{Elemental characterization of multi-Ti oxide phased nanostructure by X-ray diffraction:}

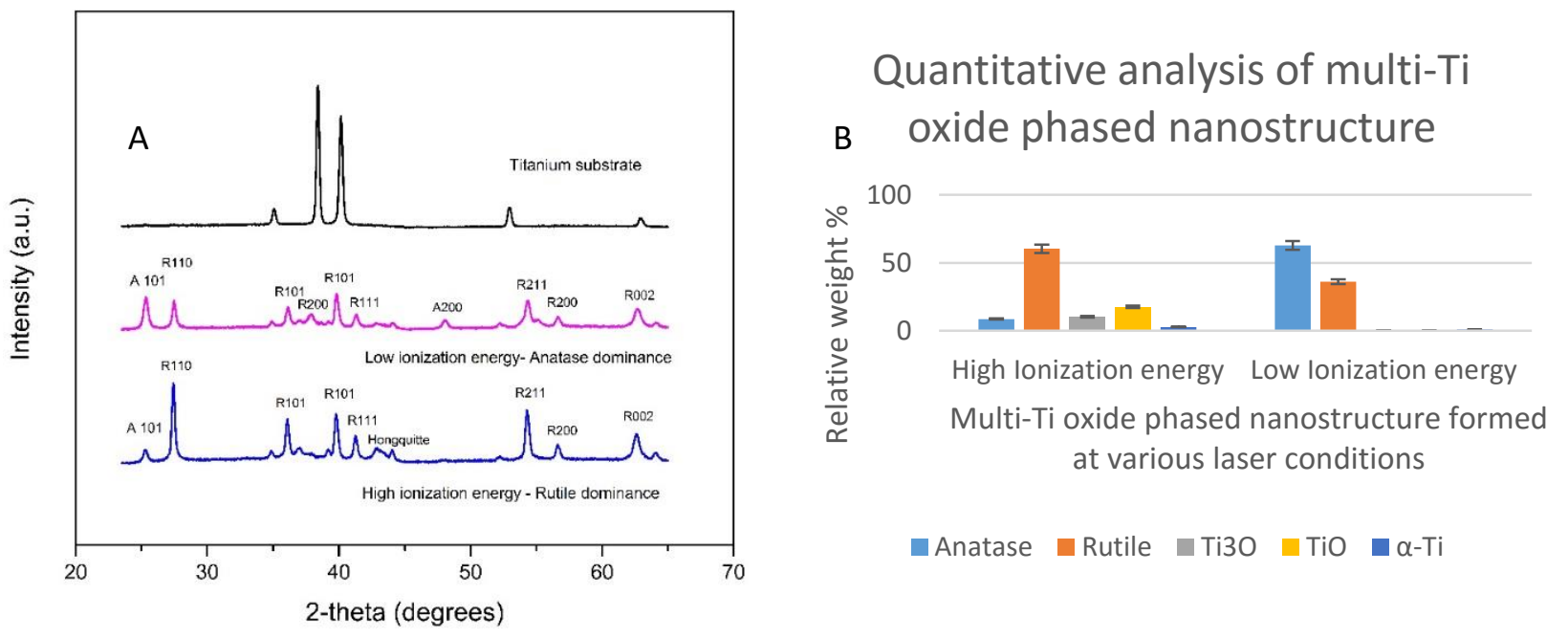

Figure 10: (A) XRD analysis of multi-Ti oxide phase nanostructres ,(B) Quantitative analysis of various phases present in the nanostructures

The elemental composition of this readily scalable multi-Ti oxide phased nano structure is quantitatively analyzed by employing XRD. This unique multi-Ti oxide phased nano structure 
consist of tetragonal $\mathrm{TiO}_{2}$ (anatase and rutile) and cubic $\mathrm{TiO}$ (Hongquiite) unprocessed titanium substrate is entirely composed of alpha-phase titanium. In the previous section, a mixtured proportion of these crystalline phases was observed by varying the ionization energy of the ultrashort pulsed laser. The random orientation of these crystal structures could be attributed to the varying plume mechanism and available oxygen ${ }^{63}$. From the figure $3(\mathrm{c})$, it observed that this multi-Ti oxide phase nanostructure has apparent titanium core and shell morphology; this shell is made up of multiple titanium oxides revealing the presence of anatase, rutile, trigonal oxide and non-stoichiometric cubic $\mathrm{TiO}$ phases. As earlier discussed in TEM-EDX analysis indicates there is a variation of titanium and oxygen peaks proportional, the vapor condensation formation mechanism for anatase dominance nanostructure and rutile dominance nanostructure. The ratio of titanium and oxygen formation on this multi-Ti oxide nanostructure relates directly to high ionization energy, which is achieved at high pulse fluence and shortest pulse to pulse separation time, and similarlily for low ionization energy is achieved low fluence and longest pulse to pulse separation time. As noted above as the peak power reduces with either increase of decrease in pulse to pulse separation time, anatase phase is dominant to rutile phase. However only with the combination of high peak power, and the shortest pulse to pulse separation time conditions is rutile phase dominant to anatase phase and accompanied with the presence of non-stoichiometric cubic TiO phase. Also at high peak power, and the high longest pulse to pulse separation time conditions, rutile phase is dominant to anatase phase but there is no presence of rare titanium oxide phases (trigonal cubic TiO, non-stoichiometric cubic TiO). Thus varying the pulse to pulse separation time at high peak power condition results in formation of rare titanium oxide phases. The role of these multi-Ti oxide phased nanostructures was further probed in the following section to study the potential correlation in reducing cancer cell differentiation and proliferation. 


\subsubsection{Decreased HeLa and NIH3T3 cell adhesion on multi-Ti oxide phased nano structure:}

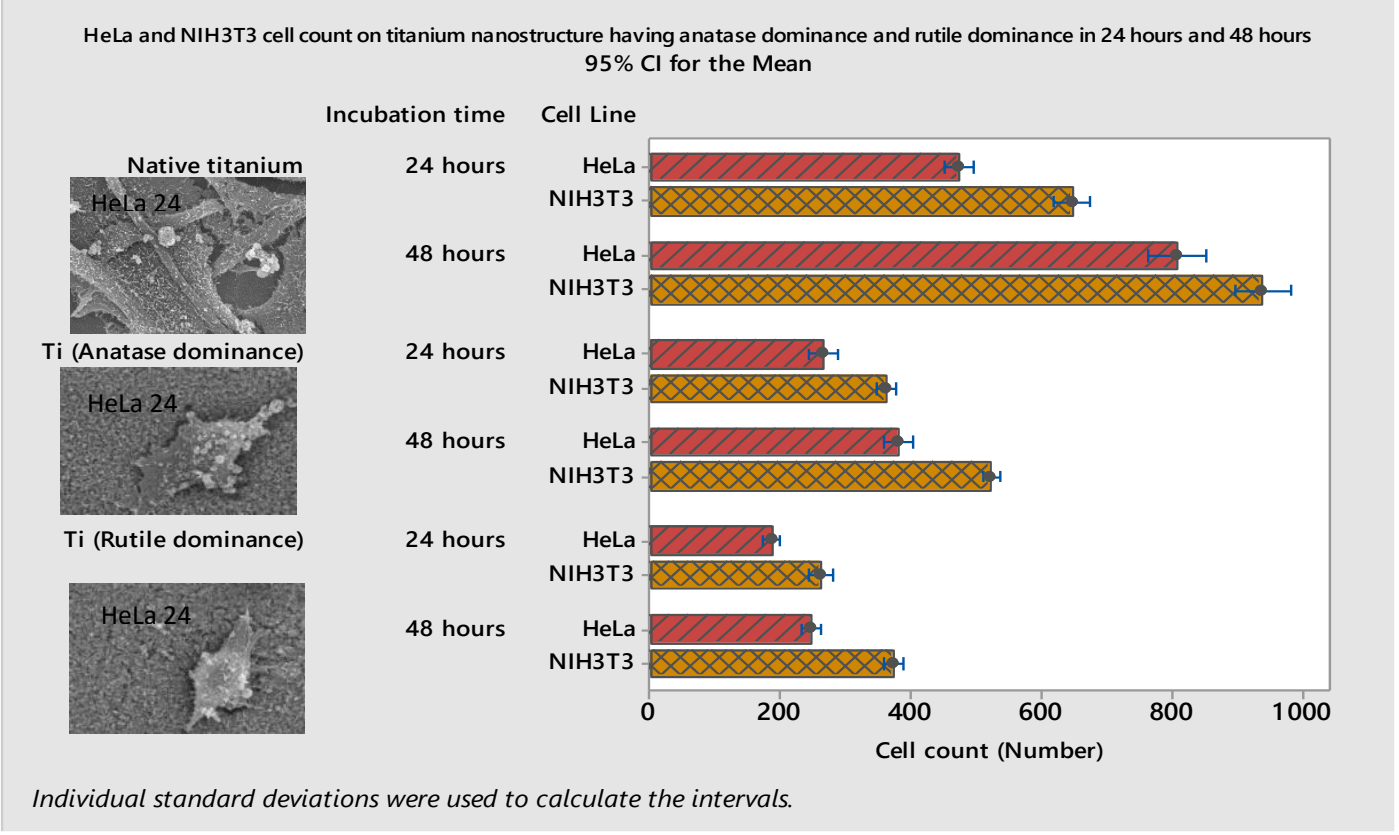

Figure 11: HeLa cell and NIH3T3 cell adhesion on anatase dominance and rutile dominance nanostructure at 24 hours and 48 hours

The number of HeLa cell and the NIH3T3 cell adhesion on anatase dominant and rutile dominant nanostructure was investigated after incubation for 24 hours and 48 hours period. The cells cultured on smooth native titanium material was used as control. To investigate if the anatase dominance, rutile dominance of multi-Ti oxide phase nanostructure reduces the HeLa cancer cell adhesion or supports survival of the NIH3T3 cell, we have quantified the total number of cells adhered onto the multi-Ti oxide phased nano structure at the end of 24 hours and 48 hours. The total number of the HeLa cell and the NIH3T3 cell adhered on rutile dominant multi-Ti oxide phased nanostructure is greatly decreased when compared to anatase dominant multi-Ti oxide phased nanostructure at the end of 24 hours (Figure 6(a) and 6(e)). But this reduction in cell adhesion was observed for both the HeLa cell and theNIH3T3 cell when they interact with the rutile dominant multi-Ti oxide phased nanostructure which indicates that these cell lines are susceptible to this dominant multi-Ti oxide phased nanostructure. These results substantiate the theory that cells sense changes in their environment and react via transmitting extracellular signals to the nucleus ${ }^{65}$.However this order of magnitude decrease in the HeLa cell adhesion when compared to the fibroblast cell indicates that these fibroblast cell is less susceptible to anatase 
dominant multi-Ti oxide phase nanostructure, when compared to rutile dominant nanostructure. At the end of 48 hours, the HeLa cell and the NIH3T3 cell we observed an up surged cell proliferative characteristics when they interact with anatase dominant nanostructure when compared to rutile dominant titanium nanostructure (Figure 6(b), 6(d), 6(f) and 6(h)). This outcome was the consequence of multi-Ti oxide phased nanostructure losing their efficacy in controlling the cell proliferation. But both the HeLa cell and the NIH3T3 cell proliferation in 48 hours was amassed in anatase dominance nanostructure, when compared to rutile dominance nanostructure which divulge that these rutile dominance nano structure are intrusive in controlling the cell proliferation. It could be speculated that at the rutile dominant multi-Ti oxide phase nanostructure the polymerization of actin filaments in the HeLa cell was adversely affected when compared to the anatase dominant multi-Ti oxide phase nanostructure. Earlier Jung lu et al., reported that there is improved cellular adhesion when they interact with the nanostructure surfaces which is achieved through by varying the height of the nanostructure ${ }^{66}$. Also Popat et al., reported that there is evidence of enhanced cellular adhesion by synthesizing a novel-metal oxide titania nanotubes that corroborate our research work being the exclusive method in utilizing the dominant phases present in multi-Ti oxide phase nanostructure for modulating the cell adhesion ${ }^{17}$. 


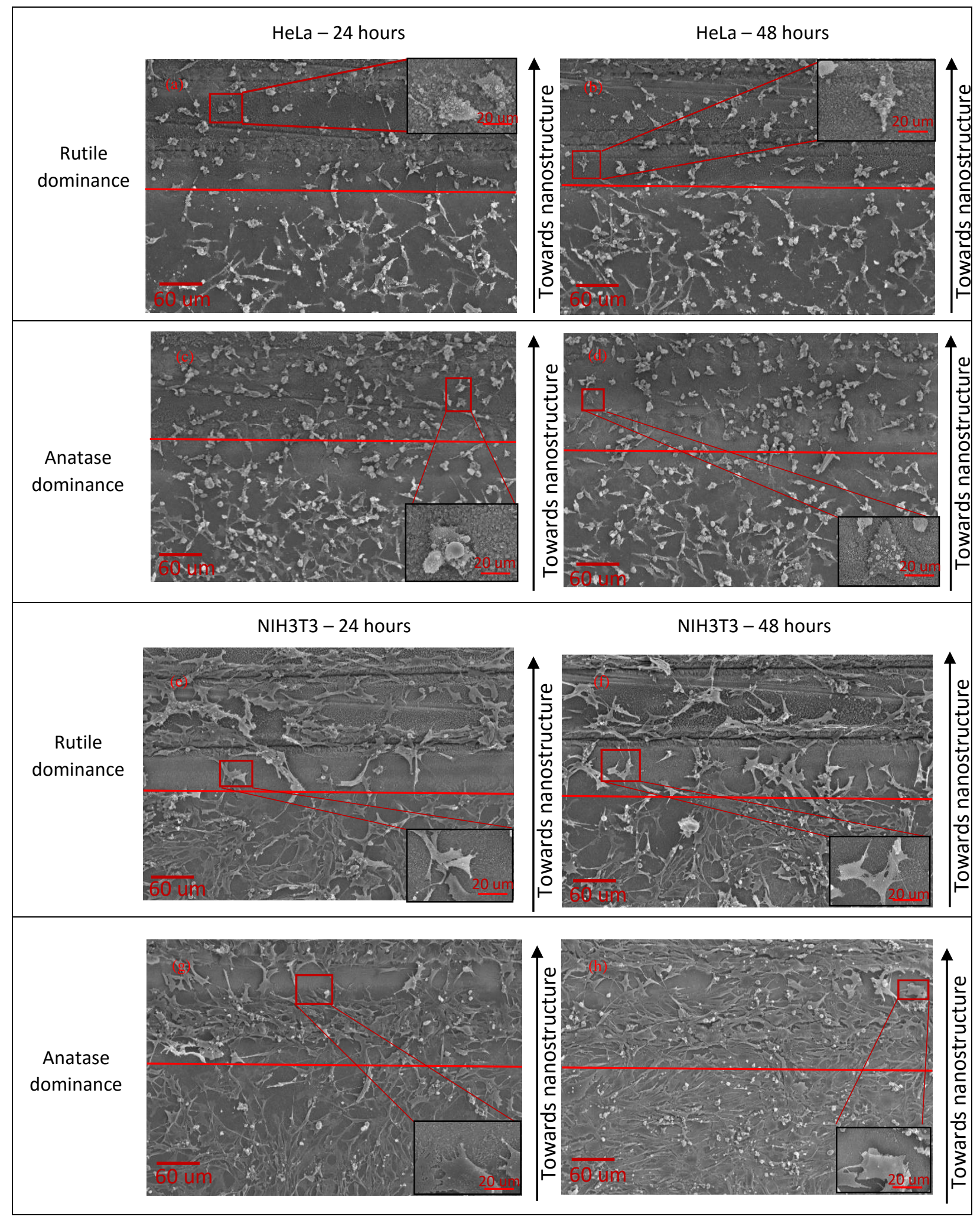

Figure 12: HeLa cell and NIH3T3 cell on anatase dominance and rutile dominance nanostructure at 24 hours and 48 hours 


\subsubsection{Modulating HeLa cell cytoskeleton behaviour when interacting on multi-Ti oxide}

phased nanostructure:
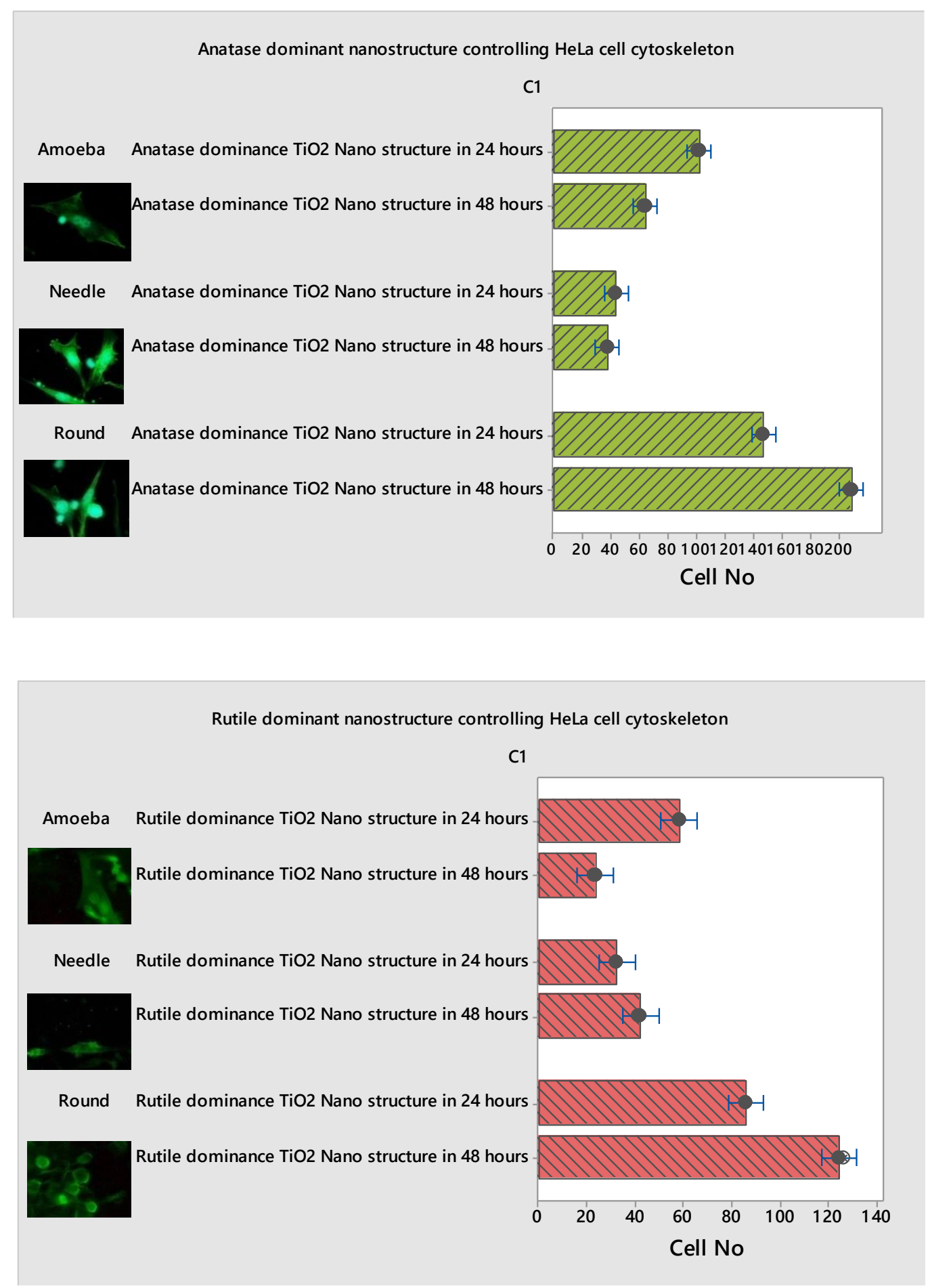

Figure 13: (1) Anatase dominant nanostructure controlling HeLa cell cytoskeletonat at 24 hours and 48 hours, (2)Rutile dominant nanostructure controlling HeLa cell cytoskeleton behaviour at 24 hours and 48 hours 
Using multi-Ti oxide phase nanostructures fabricated as described above, we are now analyzing how the HeLa cell cytoskeleton behaviour is determined under the influence of phase dominance in nanostructures. After 24 hours the HeLa cell is predominantely amoeba shaped cells (well defined) found in higher number on the anatase dominant nanostructure, when compared to the rutile dominant nanostructures. We consistently found that the proportion of needle shaped cell appeared in higher number on the rutile domiant nanostructures when compared to the anatase dominant nanostructures, which indicate that HeLa cells senses the change in material chemistry of the nanostructure favourable for them to migrate away and adhere. This behavior revealsthat the HeLa cells doesn't favor the rutile domiant nanostructure to adhere and form a well defined shape (amoeba), which is contradictory to the HeLa cell interaction with the anatase dominant nanostructure where amoeba shape cell is in amassed magnitude ${ }^{67,68}$. However to the best of our knowledge, there is no previous research work on the HeLa cell cytoskeleton behaviour can be determined by phase dominant nanostructures. Our results corroborate to our earlier result, where cell proliferation is observed more on anatase dominant nanostructure that was also observed profusively.

\subsubsection{Lack of Stress fiber on multi-Ti oxide phased nano structure:}

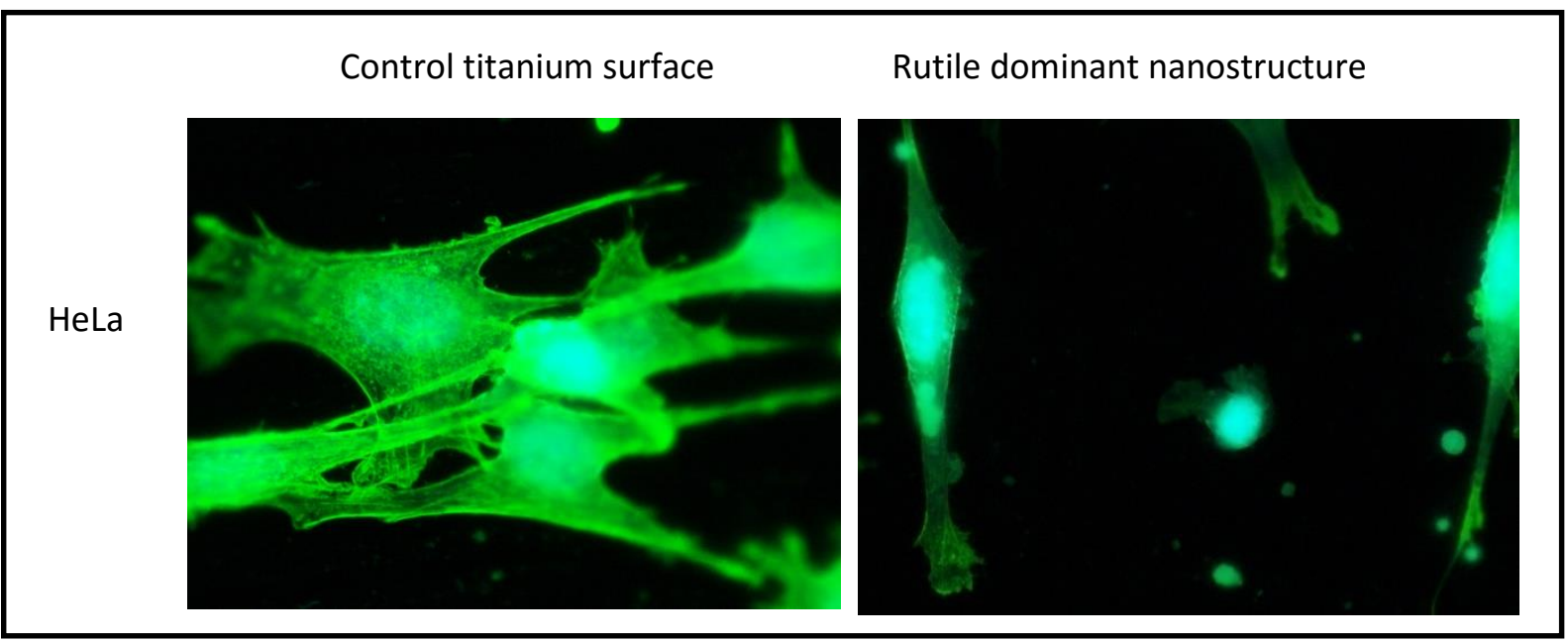

Figure 14: Fluorescent micrographs of HeLa cells on rutile dominant nanostructure and control titanium surface. Scale bar is $10 \mu \mathrm{m}$.

When the HeLa cell adhered on multi-Ti oxide phased nanostructures, they were unable to form stress fibers, which infers that there is decrease in average area of cell spread. This implies these nanostructure provides cues for cells, not to initiate stress fibers because they are not the favourable 
location for the HeLa cells to adhere and proliferate. However no initiation of stress fibers in the HeLa cell, when they interact with both anatase dominance and rutile dominance nanostructures. This indicate that the both phase dominant nanostructures has influence in reducing the stress fiber formation in the HeLa cell. Also the HeLa cells has developed highly migratory morphology having long protrusions, which is contrary to control surface where cells appeared well spread with visible stress fibers that indicate they found a favourable place to adhere. Jiyeon lee et al., stated that when the spacing between the nano rods is more than $80-100 \mathrm{~nm}$ resulting in non-adherence of cell onto the nanorods which is contrary to our research finding that the material chemistry of these multi-Ti oxide phased nanostructure results in determining actin stress fibers formation even when the distance between the nanostructure is less than $10 \mathrm{~nm}^{69}$. However this similar reduction in stress fibers was also found when the cells adhered on zinc oxide nanorods where the diameter of nanorods is approximately 50nm ${ }^{11}$. When the HeLa cell adhered on multi-Ti oxide phased nanostructures they did not have visible lamellipodial which is due to their inability to establish strong cell adhesion and thus it explains the decrease in weblike cell morphology and the increase round cell morphology on both phase dominant nanostructures ${ }^{70}$.

\subsubsection{AFM analysis of multi-Ti oxide phased nanostructure interacting with HeLa cell:}

(a)

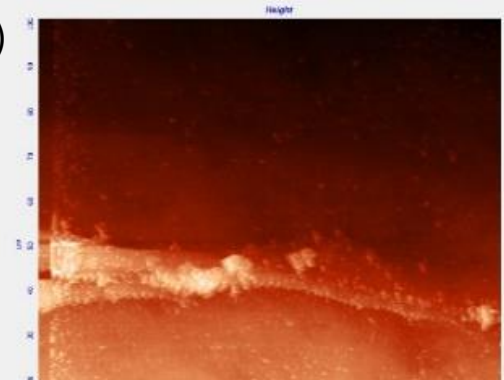

Rutile dominant nanostructure with the HeLaCell - Height

(c)

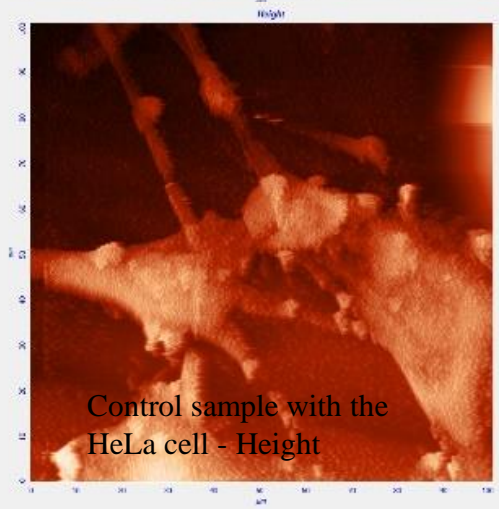

(b)

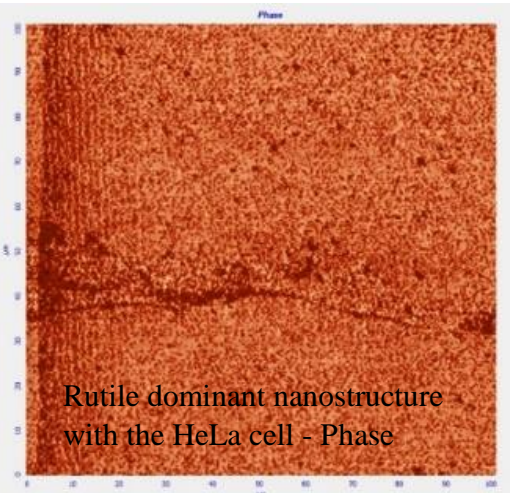

(d)

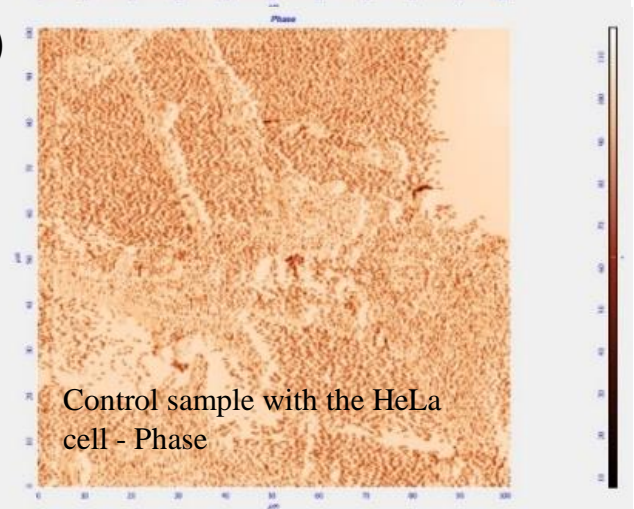


Figure 15: AFM method to analyze the height and phase contrast image of the HeLa cancer cell interacting with rutile dominant nanostructure and the control sample (a) Rutile dominant nanostructure with the HeLa Cell - Height (b) Rutile dominant nanostructure with the HeLa cell - Phase (c) Control sample with the HeLa cell - Height (d) Control sample with the HeLa cell Phase

AFM is the method that understand the extracellular structures of the cell organized at various surfaces, chemical composition and physiochemical properties of the nano structures they interact at the same time. The figure 9(a) and 9(b) provides the evidence how the HeLa cell when interacting with rutile dominance nanostructure give rise to complex phase signal shift in the sample. These phase contrast images provides a valuable information on the heterogeneity of the rutile dominance nanostructure, which is the evidence to X-ray diffraction analysis that the rutile dominance nanostructure synthesized is a mixture of anatase, rutile, trigonal oxide and nonstoichiometric cubic $\mathrm{TiO}$ phases. In figure 9(c) shows the cell adhered to the control titanium sample and cells forms a broad lamellipodia, is the evidence that the HeLa cell adhered firmly because they have found a favourable place to adhere and proliferate. In phase contrast image figure 9(d) there is no evidence of variations in material properties but in figure $8(\mathrm{~b})$ there is variations in material property. This results indicate that the HeLa cell elongates, when there is a variation in material property but a broad lamellipodia is formed, when there is no variation in material property. Thus AFM analysis of multi-Ti oxide phased nanostructure interacting with the HeLa cell is the method where the HeLa cell behavioural changes due to the change in material property can be analyzed simultaneously. 


\subsubsection{Surface area analysis of HeLa cell on multi-Ti oxide phased nano structure:}

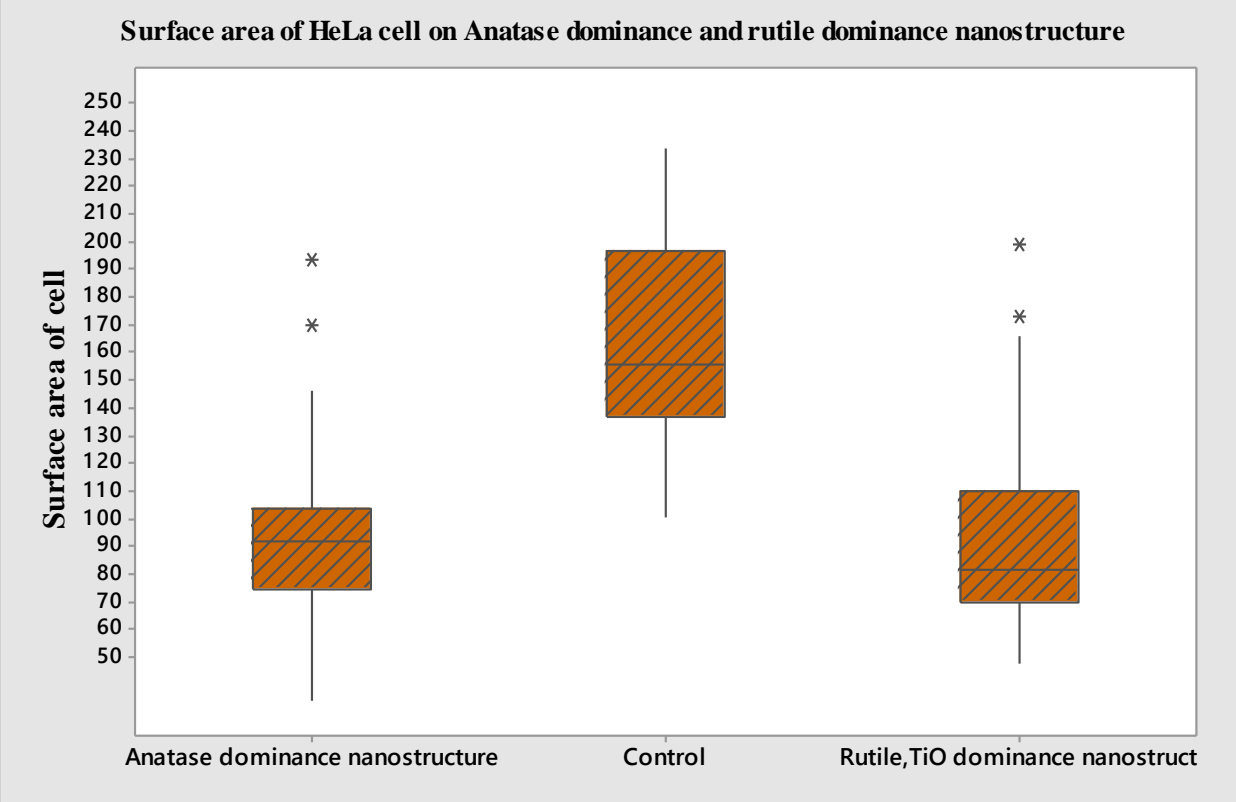

Figure 16: Surface area of HeLa cell on anatase dominance nanostructure, rutile dominance nanostructure and control titanium sample in 24 hours, units in $\mu \mathrm{m}^{2}$

From figure 16, the smooth control surface, the HeLa cell exhibited multipolar morphology with wide distribution in surface area measurement for the HeLa cell. In anatase dominance nanostructure, both amoeba and needle shaped cell was seen predominantly, when compared to rutile dominance nanostructure. Thus when composition of nanostructure is varied and applied to the HeLa cell cytoskeleton study, there is evidence in varied cytoskeleton morphology depending on the dominant nanostructure they interact (Figure 7). The quantified results for the surface area measurement of the HeLa cell is presented in the box whisker plots (Figure 10). The surface area measurement graph displayed the HeLa cell distribution midpoint (median), the first and third quartile (boxes), and the largest and smallest observation (whiskers). Results on the smooth control surface showed that the median for surface area of the cell is 160 . When subjected to rutile dominance nanostructure, surface area of cells gradually decreased and the median is 80.4. However in the anatase dominance nanostructure the surface area of cells median is 91 and this indicate the cells do not prefer to adhere on rutile dominance nanostructure. Thus most probable occurrence of surface area is high on anatase dominance nanostructure, when compared to rutile dominance nanostructure even when they both have same morphology. 


\subsection{Conclusion:}

The results added an insight into understanding the phase dominance of titanium oxides in local cellular adhesion on multi-Ti oxide nanostructure even when their individual nanostructure morphology remains the same. This unique method of fabrication using ultrashort pulsed laser where nanostructure material composition is varied but their structural morphology remained same. There were earlier studies where topography of the nanostructure has influence in regulating cancer cell proliferation. The fact that mammalian cells and cancer cells can differentiate the material composition of the nanostructure indicates that cellular functions can be modulated for targeted cancer cell therapy. 


\section{Chapter -4 \\ HeLa cell patterning by ying-yang proliferative zones using multiphase titanium oxide nanomaterial synthesized by ultrashort pulsed laser}

Cell-cell communication is mediated by various mechanical and physical cues arising from the microenvironments they interact with and the fundamental understanding of the cellular behavior aid us in designing a nanopatterning device. However there is no methods to modulate the cellular behavior using chemical cues from the nanostructure. Also there is no evidence of synthesizing ying-yang proliferative ones using varied material composition from multi-Ti oxide nanostructure. This method of fabrication ying-yang proliferative zone is applicable to HeLa cancer cell but mammalian cells does not have influence on ying-yang proliferative zones of multi-Ti oxide nanostructures.

\subsection{Introduction:}

Precise controlling of cellular microenvironments for the application of cancer cell engineering constructs, understanding the cellular interactions in diseased tissues and creating functional tissue replacements is the current problem in nanopatterning devices ${ }^{71}$. These cellular communication in microenvironments aid us to understand the influence of spatial and geometric locations of cancer cell adhesion, proliferation, and metastasis ${ }^{72}$. Thus engineering the cancer cell constructs through cellular patterning help to minimize and mimic the cellular distribution for histologic research studies. However replicating in vivo cancer cell growth environments in an in vitro model provides us an unique opportunity to study the cancer cell metastasis, their cellular interactions for the application of cancer drug screening.

There have been increased high-throughput and combinatorial biomaterial development for studying customizable efficient cellular nanopatterns for running multiple cancer drug test screening ${ }^{73,74}$. Thus nanopatterned biomaterial arrays are ideal for cellular constructs for cell-tocell, cell-to-drug and cell-o-biomaterial interactions. These engineered biomaterials mimic three dimensional environments, mechanical properties of the ECM and chemical nature of the materials that interact with the cells. Clustered, large structured integrin of the cell act as the focal adhesion and as sensors for the ECM environment at act accordingly for altering the morphology of the cell ${ }^{75,76}$.Cells responds morphologically by elongation, aligning themselves around the 
nanotopography ${ }^{77}$. The influence of topography in cellular patterning especially the depth of the grooves has more response on cellular patterning than the width or pitch of the grooves ${ }^{78}$. Clark et al., stated earlier that cells aligns to microscale grooves when they increase in depth of $1.7 \mu \mathrm{m}$ then the change in pitch of $20 \mu \mathrm{m}$. However there is no work established where cellular alignment to groove width is less than $100 \mathrm{~nm}^{78}$.

Chemical method of cellular nanopatterning has the ability to restrict cellular morphology, location of adherence, differentiation, proliferation and apoptosis of the cell. The techniques which produce cellular patterns for studying the biomolecules interaction and manipulation is micro contact $(\mu \mathrm{CP})$ printing and dry lift-off method.

The traditional and widely used method for cell patterning include micro-contact printing ${ }^{79,80}$, photolithography ${ }^{81}$, dip pen nanolithography ${ }^{82}$ and inkjet printing ${ }^{83}$. However laser based direct write technique are the only non-contact method which include laser-induced forward transfer (LIFT), absorbing film assisted laser-induced forward transfer (AFA-LIFT), biological laser processing (BioLP), matrix-assisted pulsed laser evaporation direct writing (MAPLE DW), laserguided direct writing (LG DW) for cell patterning.

Thus in this research work, we introduce a unique method to create proliferative zone and non proliferative zone nanomaterials from $\alpha$-phase titanium material using ultrashort pulsed laser. This interaction of ultrashort pulsed laser with titanium is in the femtosecond scale where both the zones created have become bio-functionalized because of various phases of titanium. This method of fabrication allows us to vary the HeLa cell cytoskeleton and the cell alignment dependent upon the nanomaterial zones they interact with. Results indicate that nanostructures formed at both low energy and high energy from the laser has both proliferative zone and non-proliferative zone. However in the proliferation zone nanomaterial anatase phase is low concentration in contrast to the non-proliferation zone nanomaterial where anatase phase is of higher concentration. This unique way of creating two different zones in one nanostructure by varying the ionization temperature in the plume during ultrashort pulsed laser interaction. 
Ultrashort pulsed laser interacting with titanium substrate

Ionization process in the laser plume resulted in the formation of various combination of titanium oxides depending upon the energy from laser
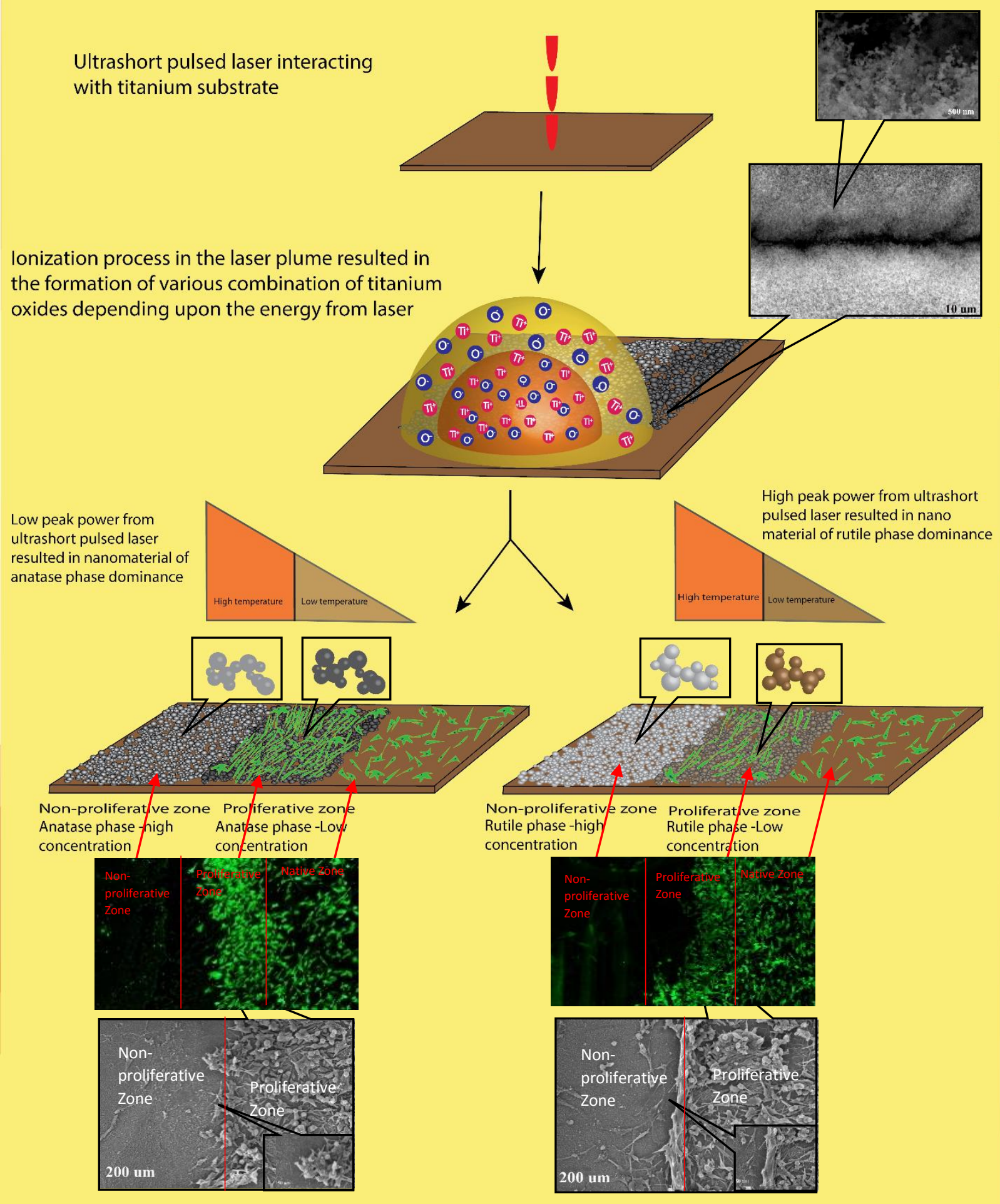

Figure 17: Graphical abstract illustrating ying-yang proliferative zones using ultrashort pulsed laser 


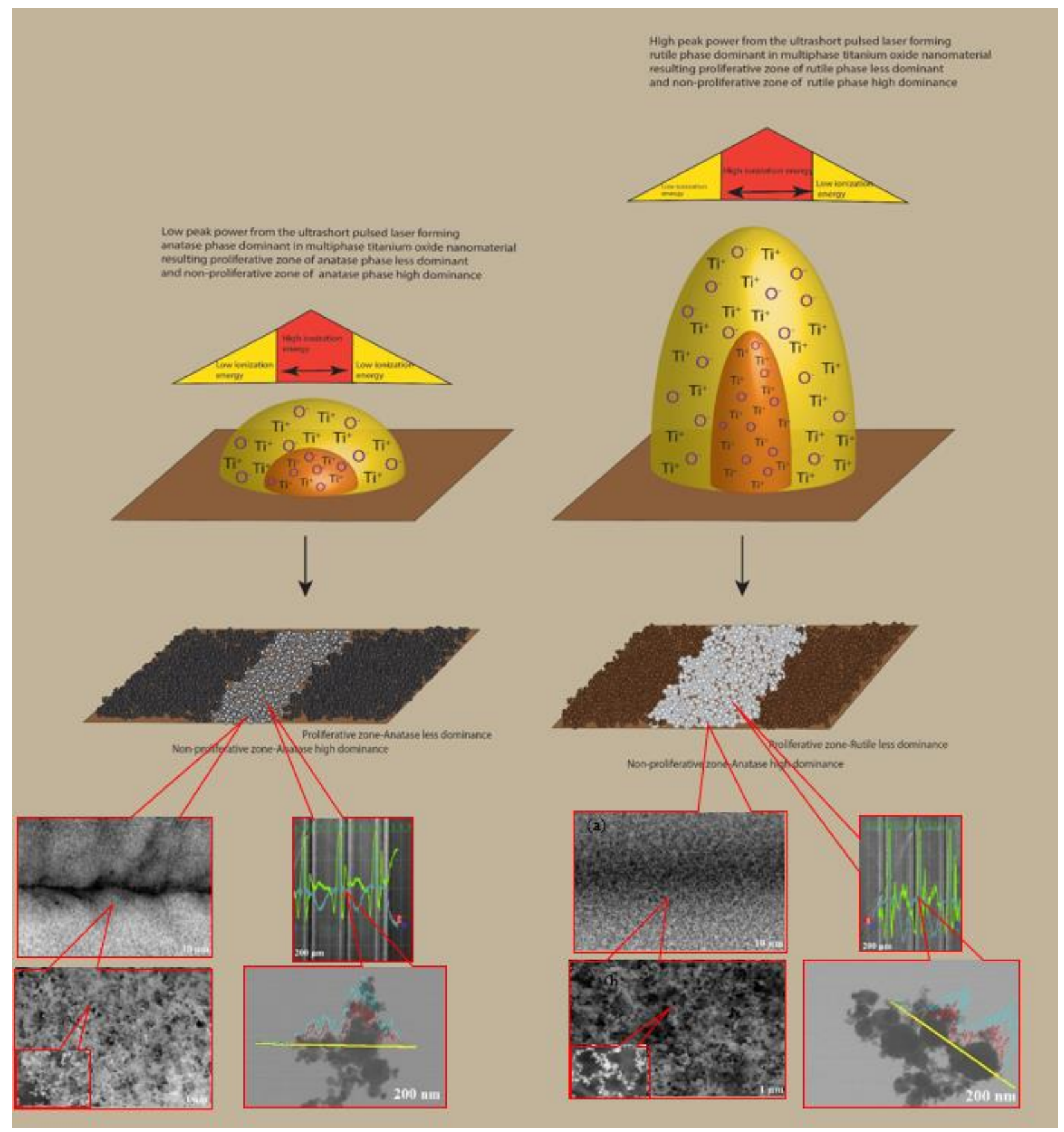

Figure 18: Multiphase titanium oxide nanostructure synthesized by varying the ionization energy of ultrashort pulsed laser, (A, B, C)-nanostructure generated by high ionization energy from ultrashort pulsed laser, (D, E, F) - nanostructure generated by low ionization energy from ultrashort pulsed laser 


\subsection{Results and discussion}

\subsubsection{FESEM with EDX analysis of nanostructures generated by varying ionization energy:}

In fig 12, FESEM image shows multi oxide phased titanium nanostructure synthesized by varying the ionization energy from the ultrashort pulsed laser. It is evident from the figure 18(A) and 18(D), the overall nanostructures synthesized by high ionization energy and low ionization energy respectively. However when ionization energy from the ultrashort pulsed laser low interacted with titanium there is evidence of trenches when compared to high ionization energy. When comparing two figures $18(\mathrm{~B}-\mathrm{E})$, it is observed clearly that trenches were formed when low ionization energy interacted with titanium, this appearance of the morphology was due to the low energy generated from the laser pulses and the pulse duration in between the pulses is longer. However this result is contradictory to nanostructures generated at high ionization energy at longer pulse to pulse separation time where there is no observance of trenches. EDX results of FESEM micrograph of the multiphase titanium oxide nanostructures results indicate that there is variation in titanium and oxygen concentration in both the conditions. HR-TEM -EDX of the nanoparticles results also confers with the FESEM-EDX results of the nanostructures. These results indicate that the influence of energy from the ultrashort pulsed laser resulted in nanostructure having a variation in titanium and oxygen concentration which corresponds phase transformation in nanostructure.

\subsubsection{AFM analysis of investigating nanostructures generated at various ionization energy:}

(a)

Height- High ionization energy

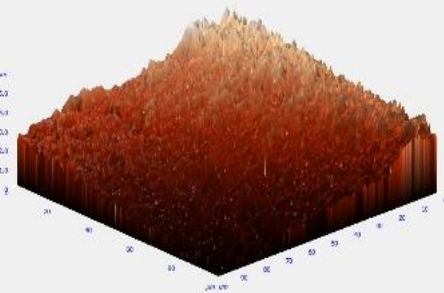

(c)

Height-Low ionization energy

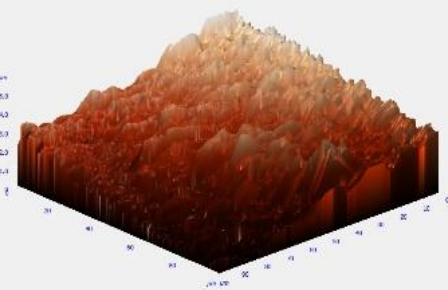

(b)

Phase- High ionization energy

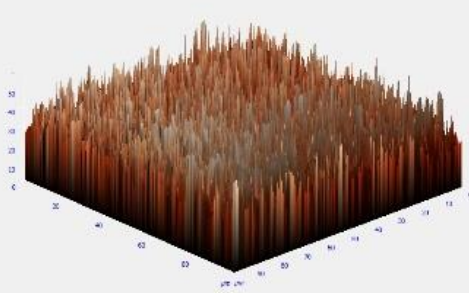

(d)

Phase-Low ionization energy

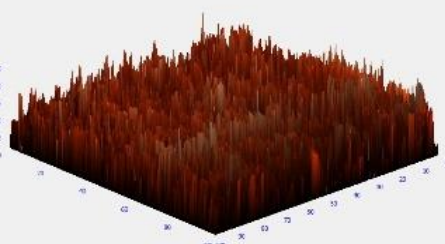


Figure 19: AFM method to analyze the change in height and material composition of nanostructure synthesized by varying ionization energy, $(\mathrm{a}-\mathrm{b})$ height and phase- high ionization energy, (c-d) height and phase-low ionization energy

AFM is the method where we can determine simultaneously the height and phase of the nanostructure. The qualitative increase in nanoscale was detected for both height and phase simultaneously when the ionization energy from the ultrashort pulsed laser interacted with titanium material. However when the ionization energy is lower we observed there is increase in height of the nanostructure when compared to the medium and high ionization energy. This result is attributed to sufficient duration in between the pulses and also to the effective number of pulses interacting with material at a constant time. Also in all the three ionization energy phase shift diagrams, we observed there is a significant enhancement in high ionization energy condition when compared to medium and low ionization energy conditions that indicate material shift is pronounced more. Wei-qiang et al., stated that changing the voltage of anodization method results only in change in morphology of the nanotube but there was no change in material composition between various sizes of nanotubes synthesized ${ }^{67}$. Thus multiphase titanium oxide nanostructure generated by ultrashort pulsed laser is the only method where nanostructure size can be modified simultaneously modifying the material composition of the nanostructure. This change in the phase shift is the result of effective number of pulses interacting with the target material is of higher magnitude resulting in material transformation from $\alpha$-phase titanium into $\mathrm{TiO}_{2}$, cubic $\mathrm{TiO}$ which is further analyzed through XRD-analysis.

\subsubsection{Effect of nanoparticle size by varying the ionization energy:}
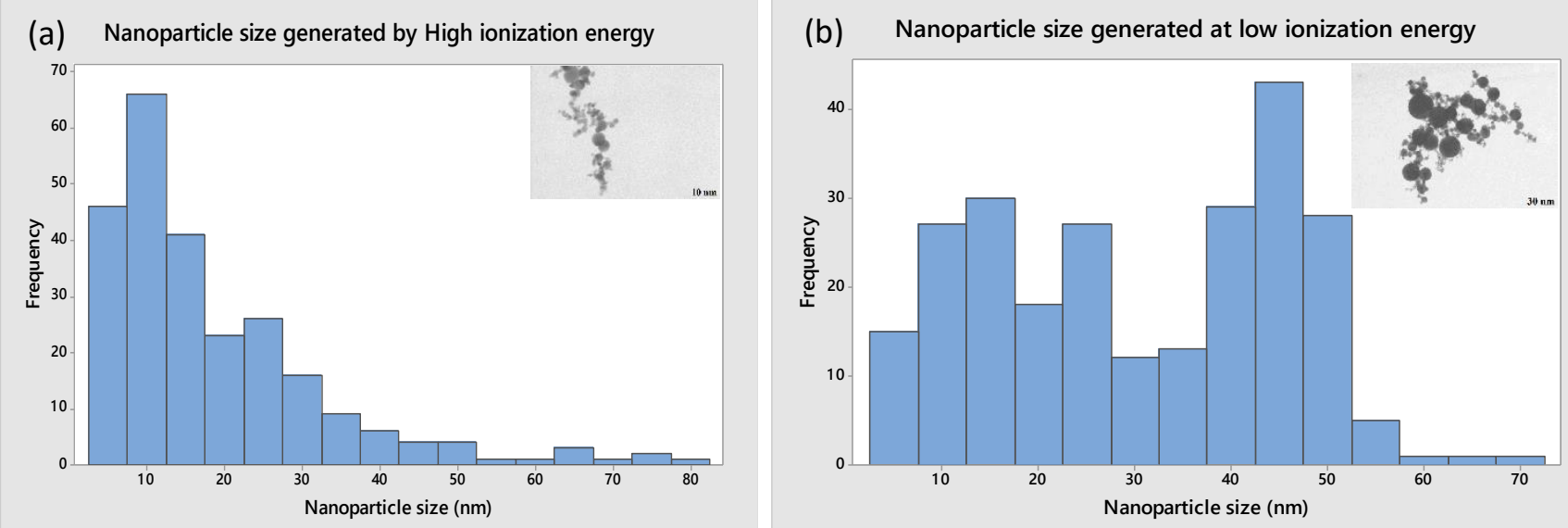
Figure 20: Nanoparticle size determined by varying the ionization energy from the ultrashort pulsed laser, (a) Nanoparticle size varied at high ionization energy, (b) Nanoparticle size varied at low ionization energy

Multi-phase titanium oxide nanostructures morphology are spherical in nature and they exhibit a highly dispersed nanoparticle sizes as observed in figure 20(A) and 20(B). When the ionization energy is high the average diameter of the spherical multi-phase titanium nano particles were about $10 \mathrm{~nm}$ however in low ionization energy conditions average nanoparticle size is $40-50 \mathrm{~nm}$. Frequency of the nanoparticle size is drastically reduced when high ionization energy is induced when compared to low ionization energy condition where we observed there is no reduction in frequency of nanoparticle size from $10 \mathrm{~nm}$ to $45 \mathrm{~nm}$. This phenomenon is attributed towards decreased laser peak power with increased pulse to pulse separation time.

\subsubsection{XRD investigation of multiphase titanium oxide nanostructure generated by varying ionization energy:}

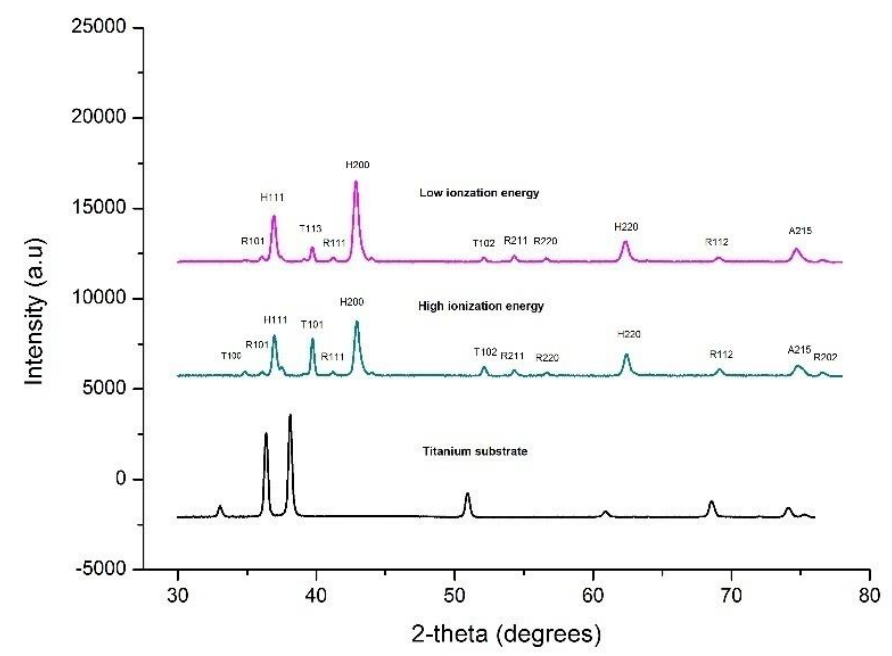

High ionization energy
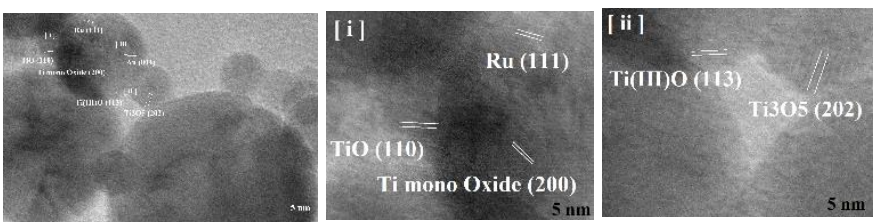

Low ionization energy
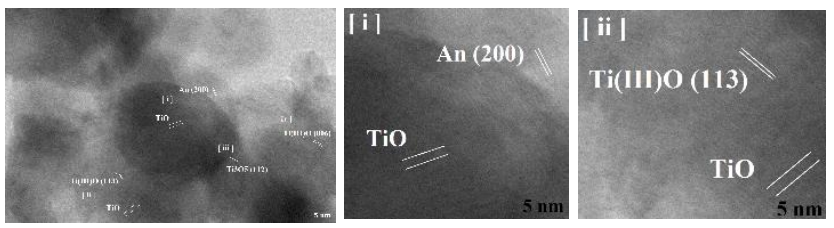

Figure 21: XRD plot of multiphase titanium oxide nanostructure synthesized by varying the ionization energy, (a) Lattice fringes of multiphase titanium oxide nanostructure generated by high ionization energy, (b) Lattice fringes of multiphase titanium oxide nanostructure generated by low ionization energy

The material composition of this multi-Ti oxide phased nano structure is quantitatively analyzed by employing XRD. This unique multi-Ti oxide phase nano structure consist of tetragonal $\mathrm{TiO}_{2}$ (anatase and rutile), cubic TiO (Hongquiite) and unprocessed $\alpha$-phase titanium substrate entirely. The random orientation of these crystal structure could be attributed by varying ionization energy 
and there is definite attribution between available oxygen for the growth of crystalline phases ${ }^{63}$. From the figure 21(c) it observed that this multi-Ti oxide phase nanostructure formed has titanium core and shell structure is made up of multiple titanium oxides revealing the presence of anatase, rutile, trigonal oxide and non-stoichiometric cubic TiO phases. As earlier discussed in TEM-EDX indicates the variation of titanium and oxygen peaks proportional in indicating the ionization energy forming anatase dominance and rutile dominance in multi-Ti oxide phased nanostructure is composed of varied anatase, rutile, trigonal oxide and non-stoichiometric cubic TiO phases. We observed earlier that as the peak power reduces it is evident that anatase is dominant to rutile phase with either increase or decrease of pulse to pulse separation time. However at high peak power condition at shortest pulse to pulse separation time rutile phase is dominant along with increase in non-stoichiometric cubic TiO. In the condition of high peak power at longest pulse to pulse separation time rutile phase is dominant but there was no dominance among the rare oxide phases between trigonal oxide and non-stoichiometric cubic TiO. Thus the role of these multi-Ti oxide phased nanostructure are further probed to study the potential correlation in reducing cancer cell differentiation and proliferation.

\subsubsection{Decrease of HeLa cell adhesion pattern on nanostructure synthesized by varying the ionization energy:}

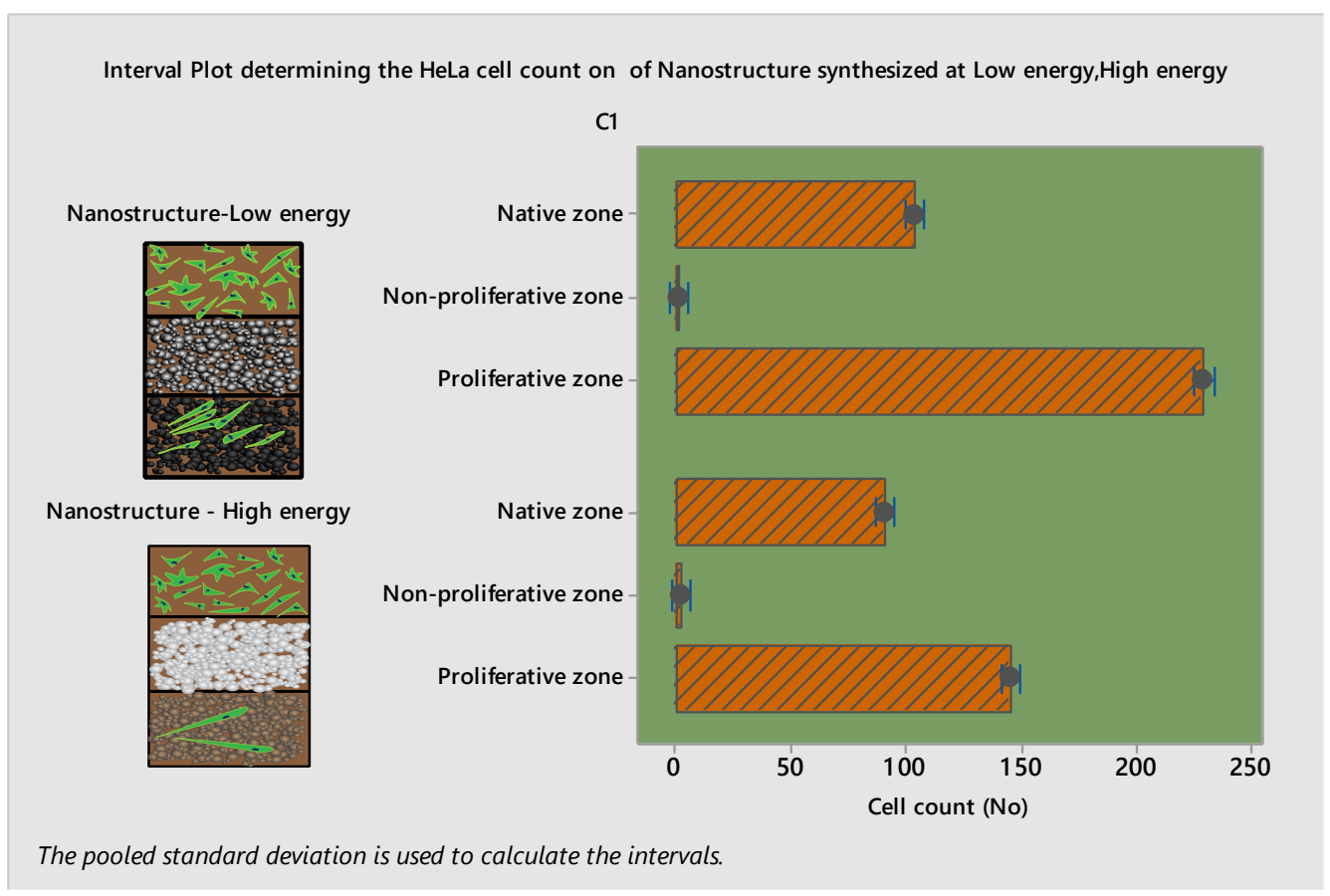


Figure 22: HeLa cell count on proliferative zone and non-proliferative zone of the nanomaterial by varying the energy from the ultrashort pulsed laser

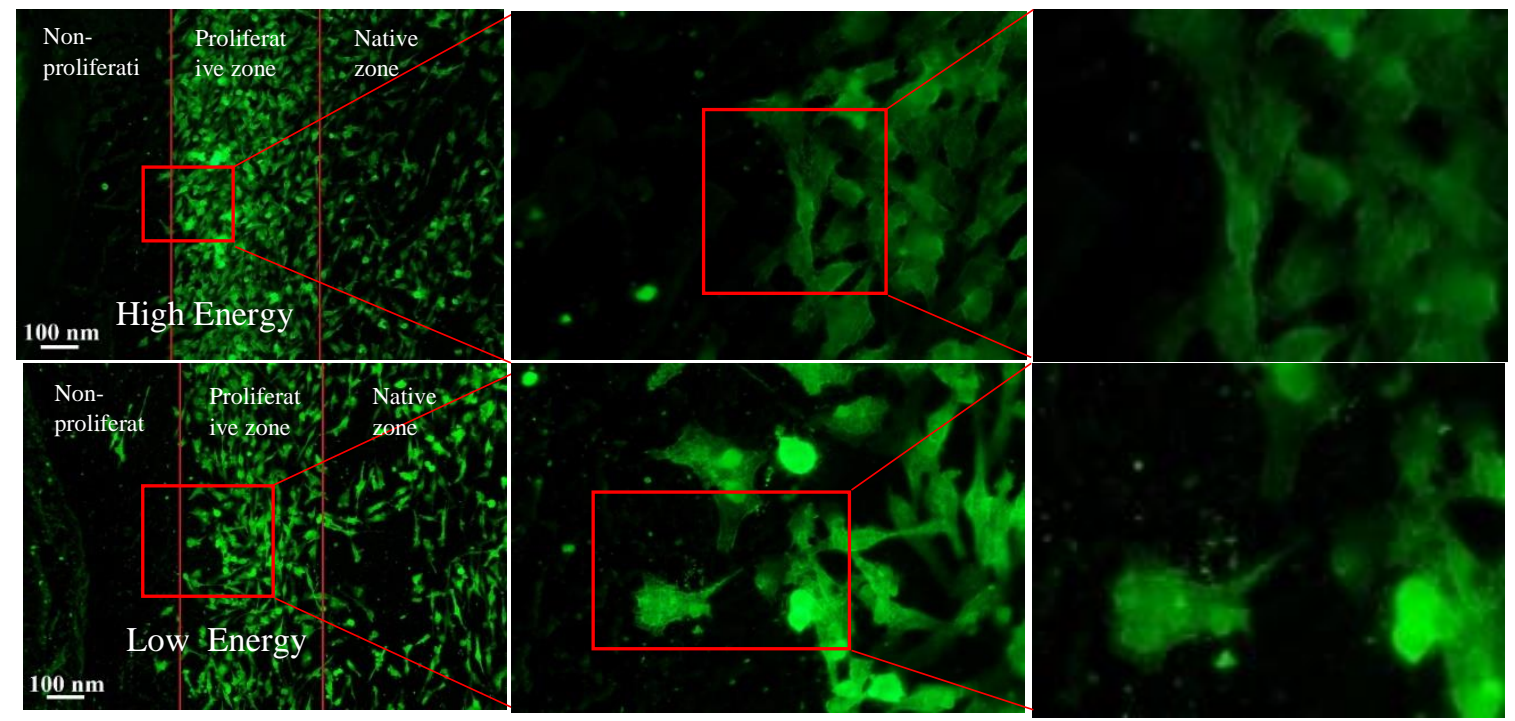

Figure 23: HeLa cell count on proliferative zone and non-proliferative zone synthesized by high and low ionization energy from ultrashort pulsed laser

The study of physical internment created by multi-phase titanium oxide nanostructures when interacting with cervical cancer cells resulted in creation of two unique zones: proliferative zone and non-proliferative zones. These two unique zones of nanostructures were synthesized by the variation of temperature difference in the plume. Nanostructures present on these two zones was likely the combination of multi-phase titanium oxides present in the nanoparticle as confirmed by HR-TEM lattice fringes analysis. However the independent composition of two unique zones cannot be identified since there is no technique available to differentiate them. From the figure 16(a) and 16(d), it is clear that there are two different zones of HeLa cell interaction with multiphase titanium oxide nanostructures observed at both low ionization energy and high ionization energy conditions. However in the proliferative zone of nanostructures formed at low ionization energy conditions we observed increased HeLa cell proliferation when compared to the proliferative zone of nanostructures formed at high ionization energy. This increased proliferation of the HeLa cell in the proliferative zone of nanostructures formed by low ionization energy was 
due to the anatase phase of $\mathrm{TiO}_{2}$ being less dominant. It is notable that the HeLa cells align in a pattern whereby we observed an increased proliferation of HeLa cells in the proliferative zone and the zone adjacent to it has become a non-proliferative zone. This similar pattern of arrangement was not observed at nanostructures formed high ionization energy where two unique conditions was observed when compared to nanostructures formed at low ionization energy : decreased proliferation of HeLa cells in the proliferative zone, decreased pattern of HeLa cells among proliferative zone, nonproliferative zone and the native zone. From XRD investigation, it is confirmed that rutile phase of $\mathrm{TiO}_{2}$ is found dominant in the nanostructure synthesized at high ionization energy condition. However HeLa cells proliferation was found comparatively decreased on the proliferative zone of the nanostructure which is the resultant of rutile phase being less dominant. To the best of our knowledge there is no nanostructured biomaterial which has the ability to create two distinct zones by varying the dominance nature of $\mathrm{TiO}_{2}$ phases for $\mathrm{HeLa}$ cells to differ differently.

\subsubsection{Proliferative and Non-proliferative zone determine the HeLa cell cytoskeleton shape:}

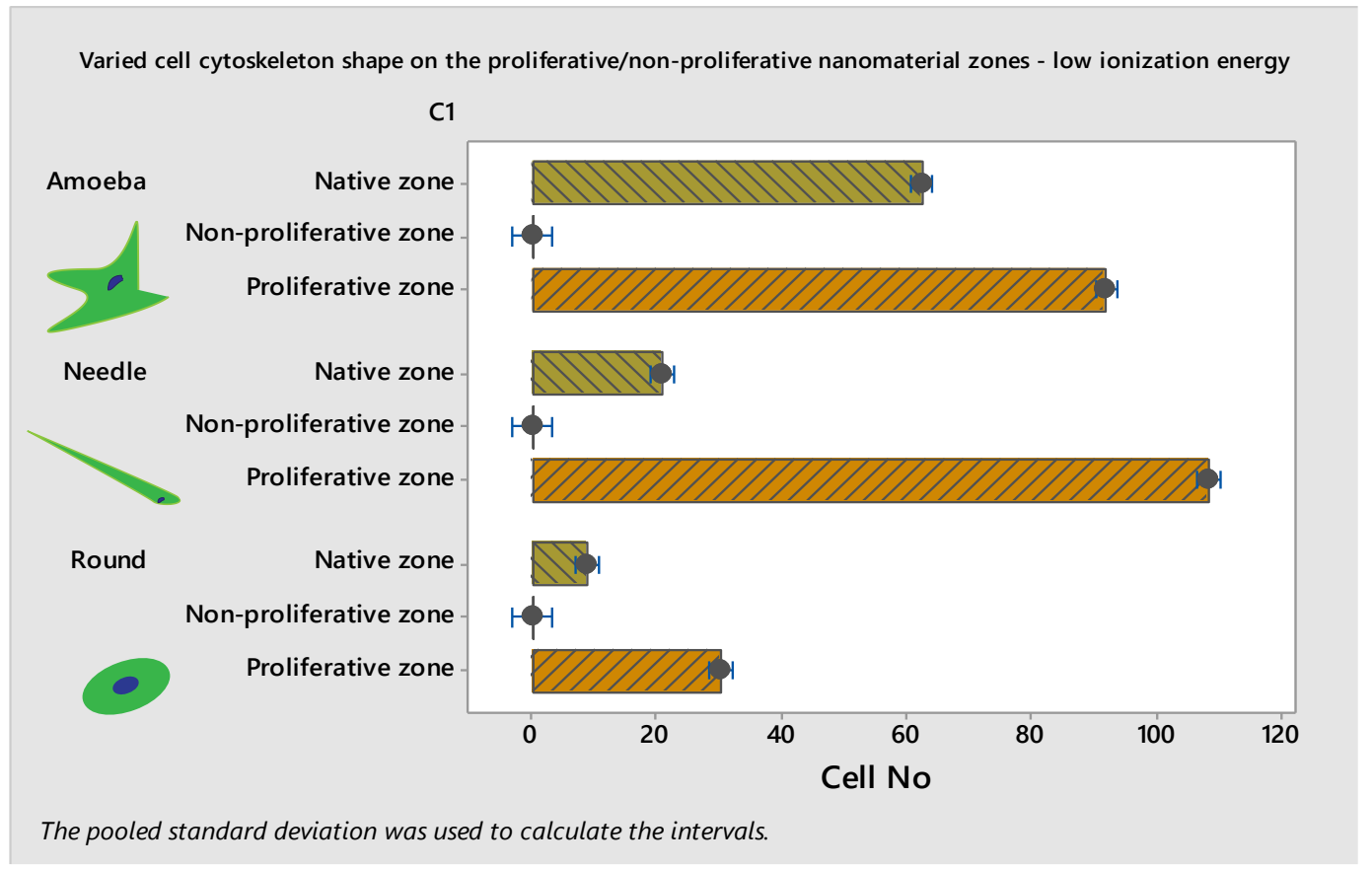




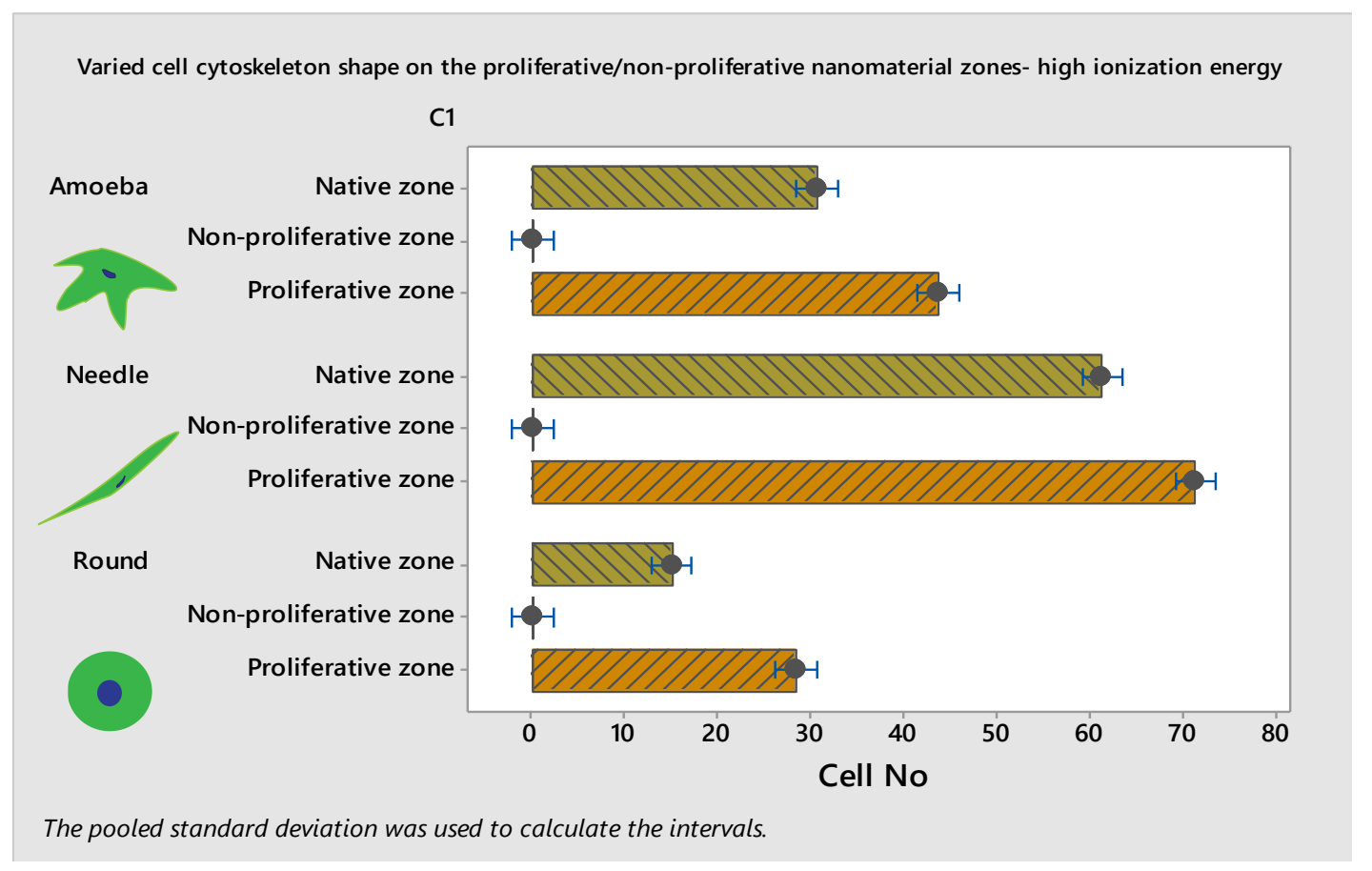

Figure 24: Influence of proliferative and non-proliferative zone of the multi-Ti oxide phased nanostructure determine the HeLa cell cytoskeleton shape

Quantification of cell morphology remaining on both proliferative zone nanomaterial and nonproliferative zone nanomaterial is evaluated using fluorescence staining at 24 hours. This analysis revealed a trend of cytoskeleton of the HeLa cell is categorized into three various shape include: Amoeba, Needle, and Round. Thus the influence of dominant phases present in titanium oxides generated by varying the ionization energy from ultrashort pulsed laser has resulted in HeLa cell interaction with the nanomaterial synthesized into either proliferative or non-proliferative zone. This analysis of HeLa cell cytoskeleton-nanomaterial interaction were confined to proliferative zone, non-proliferative zone and the native zone for comparison. After incubation time with the HeLa cell, it is observed that anatase phase being less dominant on proliferative nanomaterial zone resulted in needle shaped cell proliferated more, when compared to the rutile phase less dominant on proliferative zone which indicate that the influence of rutile phase is strenuous in controlling the HeLa cell cytoskeleton from becoming well defined amoeba shape. Interestingly, round cell morphology of the HeLa cell in the anatase phase less dominant proliferative nanomaterial appeared equivalent to rutile phase less dominant proliferative nanomaterial. However, in both dominant anatase and rutile phases of the non-proliferative nanomaterial zones conditions there is 
no evidence of HeLa cell proliferation, which indicate the dominant concentration of either anatase or rutile phases formed on the non-proliferation nanomaterial zone resulted in HeLa cell not to proliferate. Heather power et al., stated that cell cytoskeleton can be controlled by varying physical nature of the nanotopography but cannot be achieved through chemical alternation of the nanotopography ${ }^{84}$. Well defined HeLa cell cytoskeleton, amoeba is organized and appeared to be increased proliferation on anatase less dominant nanomaterial zone when compared to rutile less dominant nanomaterial zone. Increased cellular response was observed for nanostructures $13 \mathrm{~nm}$ height but when the nanostructures height is more than $95 \mathrm{~nm}$ reduced cellular proliferation is observed ${ }^{85}$. Similarly Amy brook et al., research work it is evident that geometric constraints of the structures created by micro contact printing, determines the morphology of the cell and their motility in confined environments ${ }^{86}$. Thus the earlier research work on determining the cell cytoskeleton were achieved by varying the physical morphology of the nanostructures.

\subsubsection{Various patterns of multiphase titanium oxide nanostructures determine the HeLa cell into proliferative and non-proliferative zone:}

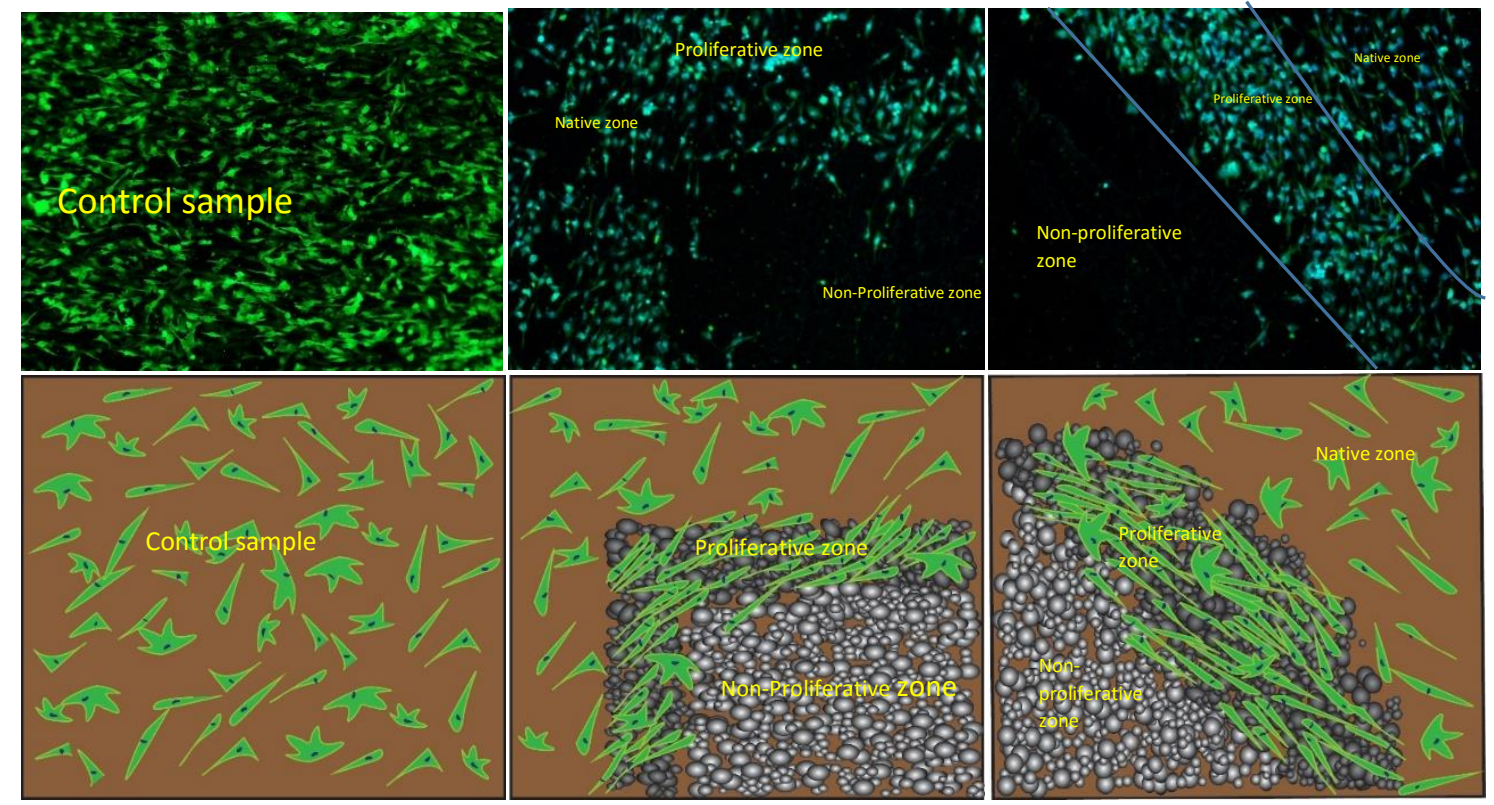

Figure 25: Influence of various nanopatterns synthesized by high energy from the ultrashort pulsed laser 
To explore the effects of various patterns of multiphase titanium oxide nanostructures synthesized by ultrashort pulsed laser when interacting the HeLa cell using fluorescence cell imaging on one dimensional confined environments. It is observed that the movement and adherence of the HeLa cell using proliferative zone and non-proliferative zone nanomaterial is an essential component for patterning devices. As shown in figure 25(A), it is clearly observed that HeLa cells doesn't adhere ,migrate into the right angled triangle and also from figure 25(B) where in the corner of circle also we found HeLa cells adhere on proliferative, non-proliferative zone. However in both the conditions we observed proliferative and non-proliferative zones which indicate that these zones were created no matter of various patterns created. Thus it proves that using ultrashort pulsed laser we can create various patterns of multiphase titanium oxide nanostructures control the HeLa cells into proliferative and non-proliferative zones.

\subsection{Conclusion:}

Ultrashort pulsed laser fabrication method is proven to be unique technique to develop nanopatterning devices that can precisely alter control adhesion, morphology, migration and differentiation. Considering previous research there is evidence of cell can be manipulated by physical cues of the nanostructure they interact with and also there is pronounced evidence of micro contact fabrication method in patterning the cell when compared to nanopatterning devices because of only their easy fabrication method. The finding of this study will definitely enhance the knowledge of fabricating nanopatterning device which can definitely influence HeLa cancer cells rather when comparing with mammalian cells. Although majority of evidence proved that topographical modification including grooves, pits were direct the cell but their interaction with cells was not achieved focal adhesion complex of the cell is not sufficient enough to interact. 


\section{Chapter -5 \\ Cancer cell signalling under the influence of multi-Ti oxide nanostructures having dominant phases}

Cancer cells in tissues adhere and their interact via cell-cell communication and cell-extracellular matrix contacts for cancer cell metastasis ${ }^{87}$. Cells senses a change in chemical and mechanical signals present in the cellular environments and they respond by cell proliferation via biochemical responses. Recently, there is continuous research to replace the tissue with artificial scaffold using biocompatible materials that would enable the cells to response positively by proliferation behaviour. Earlier biochemically organized complex of proteins, polysaccharides of different length and physical properties are considered the naturally available ECM that play the vital role in deciding the behavior of the cell. There has been considerable amount of experimental research on manipulating the cell-cell adhesion by nanomanipulation using drug loaded techniques where cell specific drug is coated onto the surface of the nanostructures.

\subsection{Introduction:}

The approaches to modulate cell functionality are focused on controlling nanoscale adhesion either by spatial patterning or nanotopography of the substrate. The focus is now more on nanoscale surface modification of titanium which offers excellent bio integration for mammalian cells due to the creation of more surface area made available for cells to freely interact and also to adhere when compared to usage of bulk titanium material ${ }^{88}$. Inspite of numerous investigations on intrinsic and deep understanding of the factors governing adhesion of cells to nano biomaterial surfaces is still limited. It is crucial among various different effects responsible for cell behaviour, related to viability, proliferation, motility, adhesion, morphology, cytoskeleton arrangement by controlling the surface chemistry while keeping the same nano morphology. In particular, designing an effective nano surface chemistry preventing $\mathrm{HeLa}$ cancer cell proliferation has remained a fundamental challenge which is overcome through synthesizing readily scalable oxidised titanium nano bio-material. 
The directional single cell alignment is due to varied cues arising from the substrate which plays a vital role in dictating the migration pattern of grouped cells. Aligned cells were elongated near the buffer zone of nanostructure when compared with cells cultured on the smooth control surface sample. Previous studies on directional cell alignment have concentrated on substrate with topographical changes. However the functionalization of "engineered polar-neoplastic" nanostructure enable us to align the cell and reducing the proliferation of cell altogether. Therefore directional alignment of cell is attributed to the presence of varying phases of $\mathrm{TiO}$ and $\mathrm{Ti}$ present in the nanostructure. Moreover when HeLa cancer cells interact with nano bio-material formed at low peak power in longer pulse to pulse width they appeared to be not aligned, remained to have stable cell adhesion to the nanostructure. But at high peak power at shorter pulse to pulse width these neoplastic HeLa cancer cell are aligned with poor cell adhesion and ensuing apoptosis explains the reason for the significant decrease in cell number. Thus the surface chemistry of titanium nano biomaterial can provide deeper insight into the mechanism of cell-repelling when they come in contact with nano biomaterial and leads us to design an innovative non-drug induced altering cell behaviour.

When cells comes in contact with the biomaterial they would perceive the chemistry of surface of the biomaterial using their integrin membrane protein for finding the suitable sites for adhesion and differentiation. It is speculated that the filopodia present on the surface of the cells acts as a main sensory tools for cells to find the preferential sites for cell adhesion and thereby initiating different distinct morphologies such as static and motile. Static cells are one which has the morphology of well-established focal adhesion with many contractile stress fibers pulling the cells flat. Whereas motile cells have a rounded body with leading edge (lamellipodium) and a tail with many filopodia ${ }^{89,90}$. These cells move to the desired site it is achieved by G-protein signalling and actin cytoskeleton. The Rho sub-family of G-protein induces the actin contractile stress fibers to assemble the cell pull against the substrate whereas the Rac induces the lamellipodium and Cdc42 is required for filopodia formation ${ }^{91}$. Therefore the cells lacking Cdc42 will migrate in random manner since they don't sense chemotactic gradients and thus it present us the evidence that filopodia involvement in cell sensing ${ }^{92}$. 


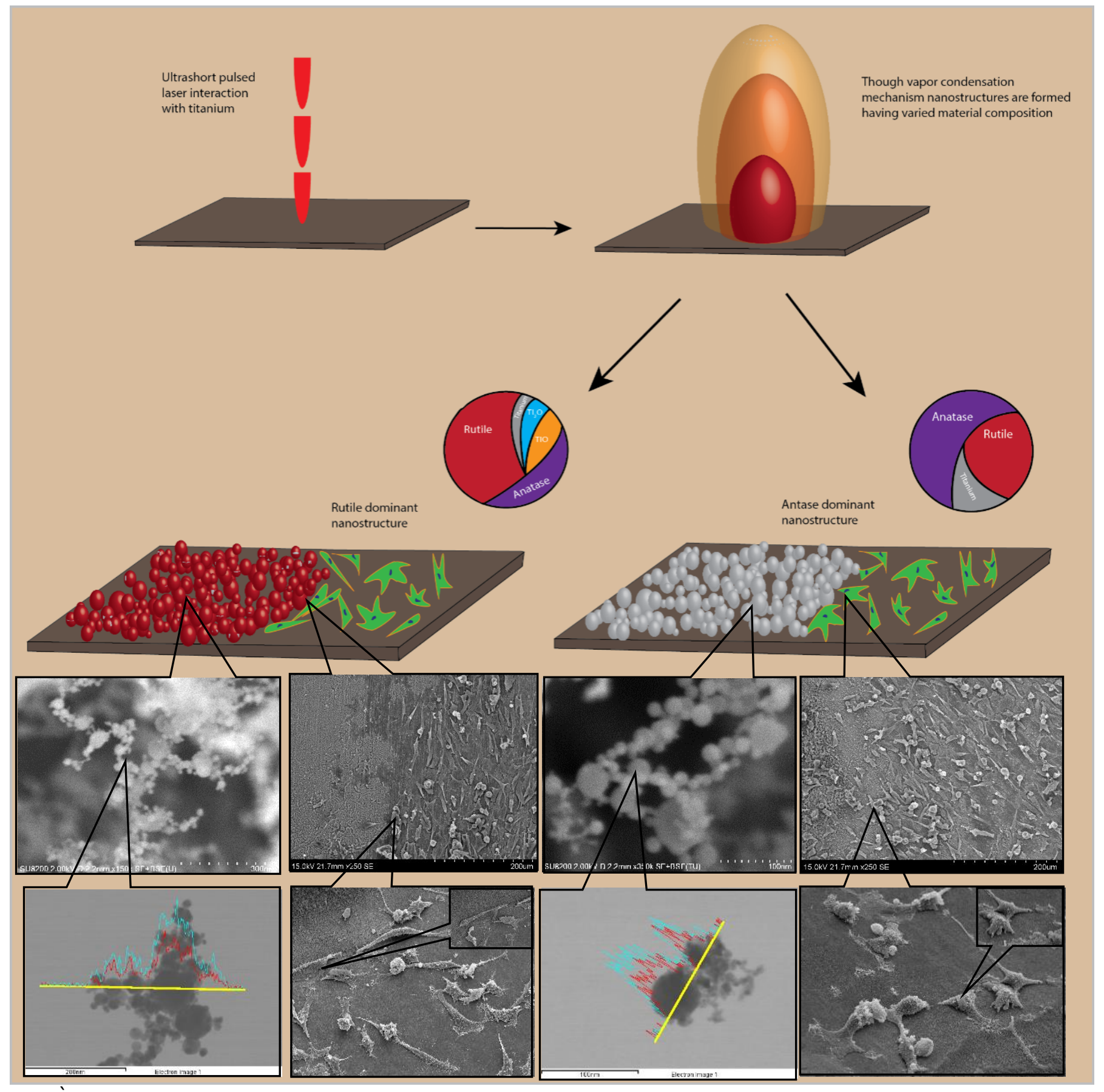

Figure 26: Graphical illustration representing the dominant phases of titanium oxide influence HeLa cell - cell signalling 


\subsection{Results and discussion:}

\subsubsection{Influence of phase dominance of titanium oxide nanostructure determines cell-to-cell signaling}

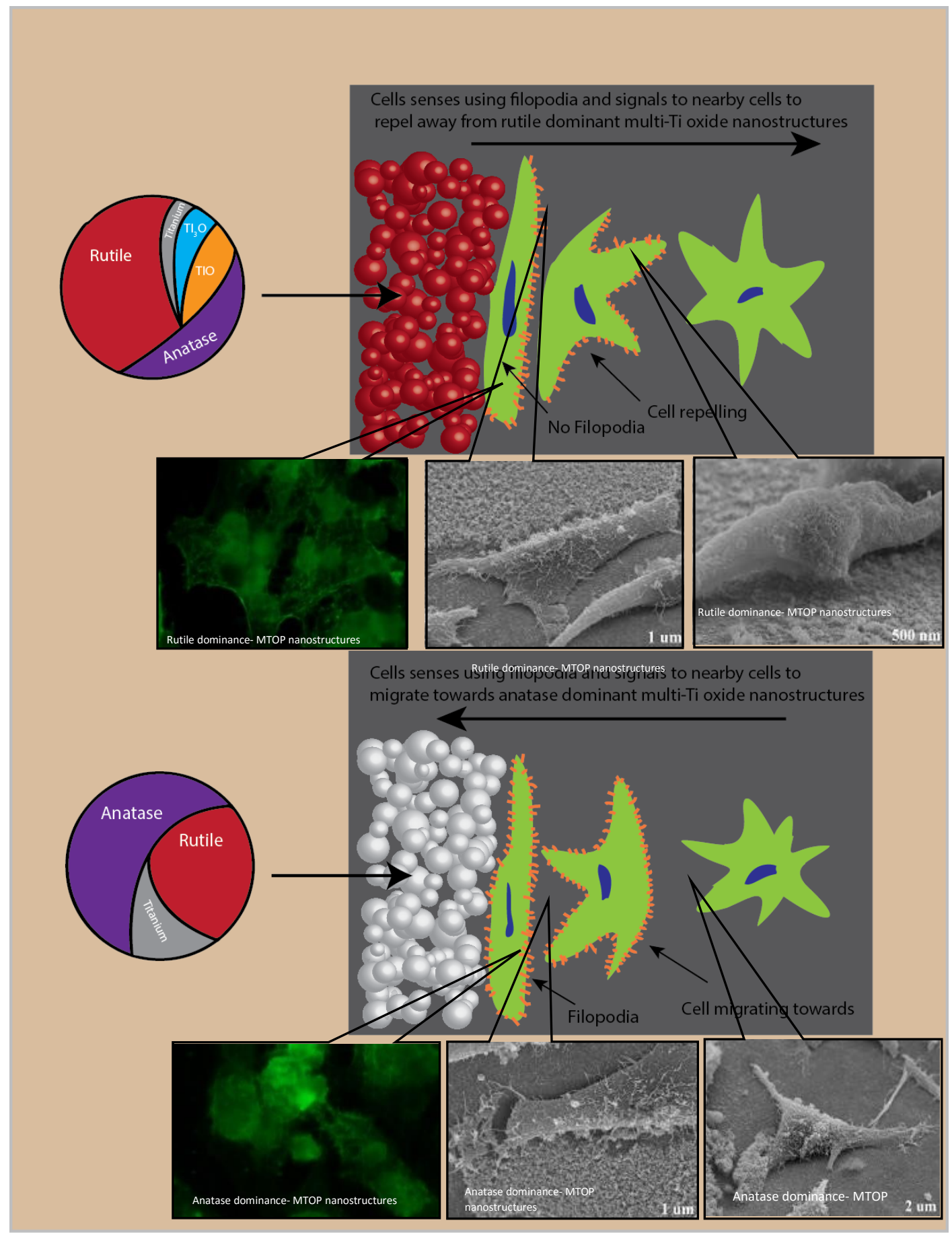

Figure 27: HeLa cancer cell-cell communication influenced by dominant phase of multi-Ti oxide phased nanostructure 
The focal adhesion, stress fiber formation of the cell are co-dependent on each other for governing cell shape and cell movement at nanoscale due to spatial distribution of integrin clusters ${ }^{93}$. We evaluate the formation of focal adhesion point formation at 24 hours of incubation when the cells are exposed to both anatase dominance and rutile dominance titanium nanostructure. Figure indicate that cells near the nanostructure initiate filopodia depending on the dominant nature of the nanostructure they interact with. This intricate cell signalling is the fundamental in understanding the cell-biomaterial interaction. We have found that the material composition of the nanostructure has influence in determining the cancer cell to cell signalling which provides the fundamental understanding for cancer therapy. Alexander et al., stated the micro patterned structural morphology have the ability for cell-cell communication to happen ${ }^{94}$. However earlier studies indicate that notable cell- cell signalling happens, but does not result in low adhesion of fibroblast cells onto nanotopography ${ }^{95}$. It is observed that needle shaped cytoskeleton cells are predominantly form near the phase dominant nanostructures. When cancer cells interact with anatase dominant multi-Ti oxide nanostructures resulted in unique cytoskeleton behaviour of the cell where broad lamellopodial is formed at the edge of the needle shaped but when compared to rutile dominant nanostructures interaction where there is no presence of lamellopodial on the needle shaped cell. This indicate the individual cell signalling happens which indicate the interaction with the phase dominant nanostructures can play a vital role determining the cytoskeleton behaviour of the cell. There were evidence that three-dimensional microenvironments of the nanostructures have the ability to reducing differentiation but no evidence of polarity of the cell can be changed ${ }^{96}$. This indicate that the morphology of the nanostructures synthesized have the ability of reducing the differentiation but not able to regulate the cellular behaviour. 


\subsubsection{Mechanotransduction mechanism by which cellular elongation is determined by the}

\section{influence of multi-Ti oxide phased nanostructure:}

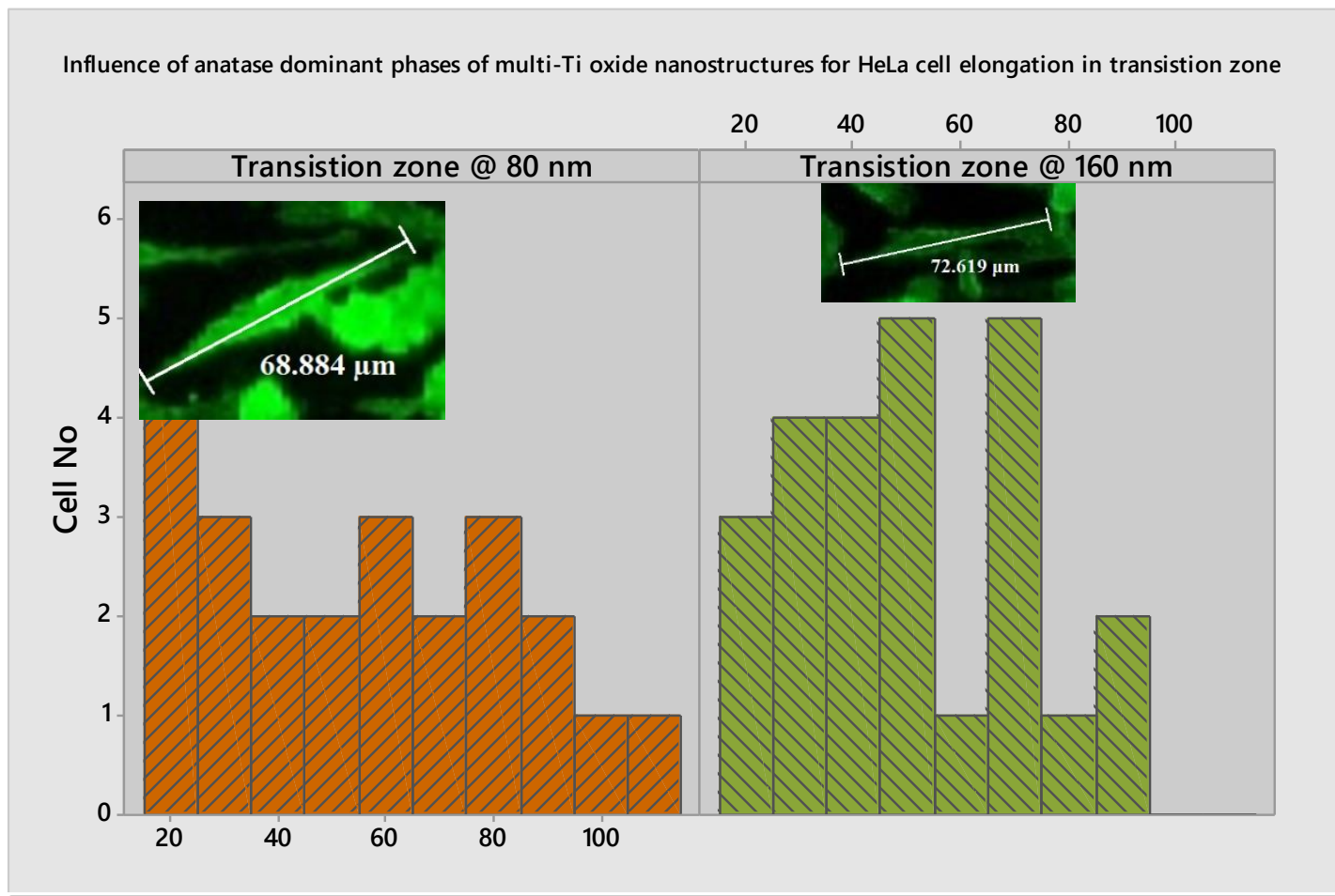

Influence of rutile dominant phases of multi-Ti oxide nanostructures for HeLa cell elongation in transistion zone

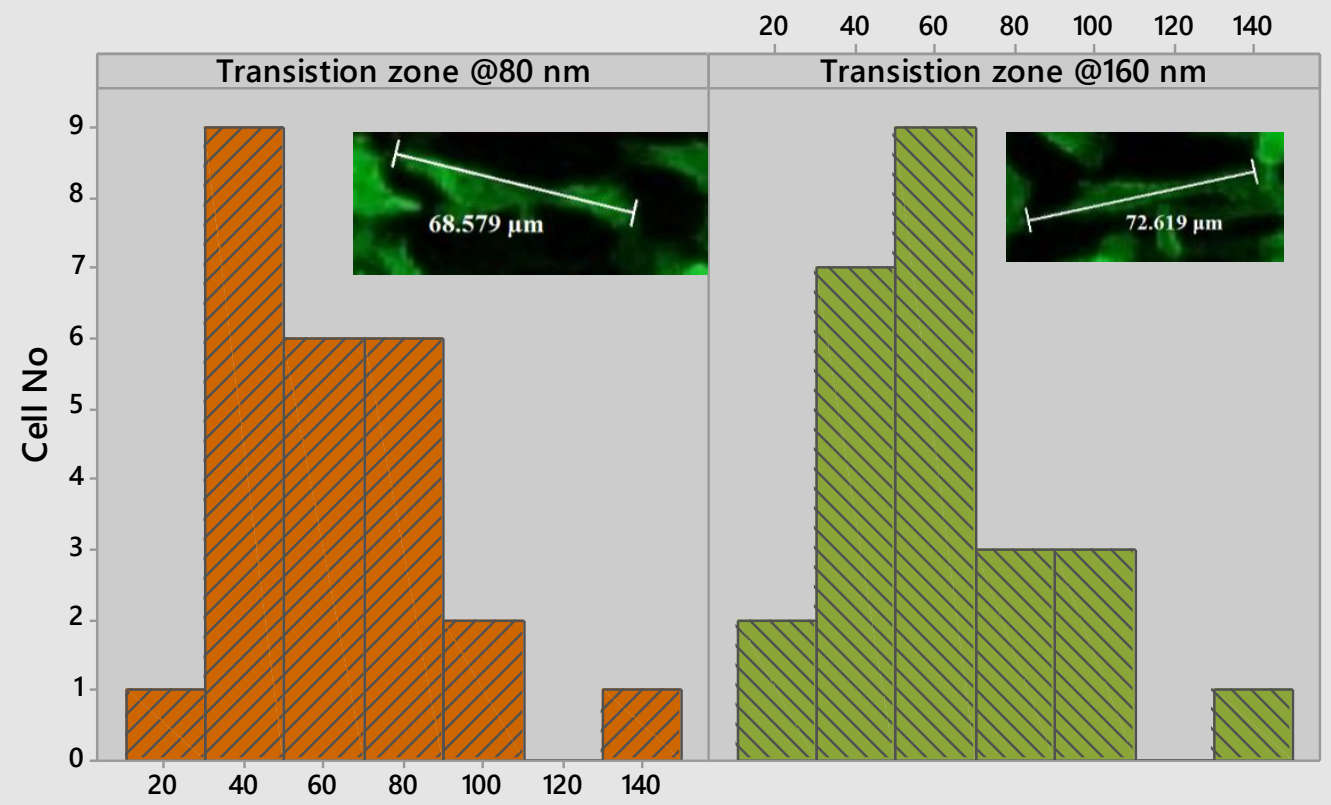

Figure 28: Displaying the influence of dominant phases of multi-Ti oxide nanostructure in determining HeLa cell elongation at 24 hours 
The sensitivity of mammalian cells to nanoscale variations is offered by spacing in between the nanoparticles on the substrate ${ }^{97}$. Studies reveal that cell respond to the gradient of $15 \mathrm{~nm}$ per mm provides a gradient having a spacing of 58-73nm. However cell elongation was achieved only in the direction of gradient and they have strong tendency in determining the direction of cell migration. There is no evidence of earlier research that material composition of the nanostructure has influence in determining the HeLa cancer cell elongation. Also, there is not enough research work on multiple phases in a single nanostructure have the ability to controlling the cellular cytoskeleton behaviour. In this research, we have demonstrated a unique opportunity where the HeLa cancer cells elongation can be modulated by varying the dominant phases present in a multiTi oxide nanostructure. At high ionization energy from the ultrashort pulsed laser results in synthesizing rutile phase dominant in multi combinatorial titanium oxides phases results in cell elongation more predominately when compared to low ionization energy interaction with titanium resulted in anatase phase dominant in multi combinatorial titanium oxides where HeLa cancer cell elongation is lesser. From the figure and, where transition zone at $80 \mu \mathrm{m}$ for both rutile and anatase dominant phase it is observed that total length of HeLa cancer cells was maximum at $20 \mu \mathrm{m}$ and $40 \mu \mathrm{m}$ respectively. This indicate that cells on dominant rutile phase intend to migrate away from the material when compared to the HeLa cancer cells on dominant anatase phase nanostructure. However at $160 \mu \mathrm{m}$ from the transition zone it is observed that at number of HeLa cancer cells elongated on dominant rutile phased nanostructure is also predominantly higher when compared to dominant anatase phased nanostructure. This also indicate that elongation of HeLa cancer cell is higher of dominant rutile phased nanostructure is more when compared to dominant phased nanostructure. In both the transition zone $80 \mu \mathrm{m}$ and $160 \mu \mathrm{m}$, number of HeLa cancer cells on $160 \mu \mathrm{m}$ transition zone there is reduced number of cells when compared to $80 \mu \mathrm{m}$ transition which infer that the concentration of dominant phases of multi-Ti oxide nanostructure losses their efficacy. 
5.2.2. Directed HeLa cell and NIH3T3 cell repelling away from multi-Ti oxide nanostructure:
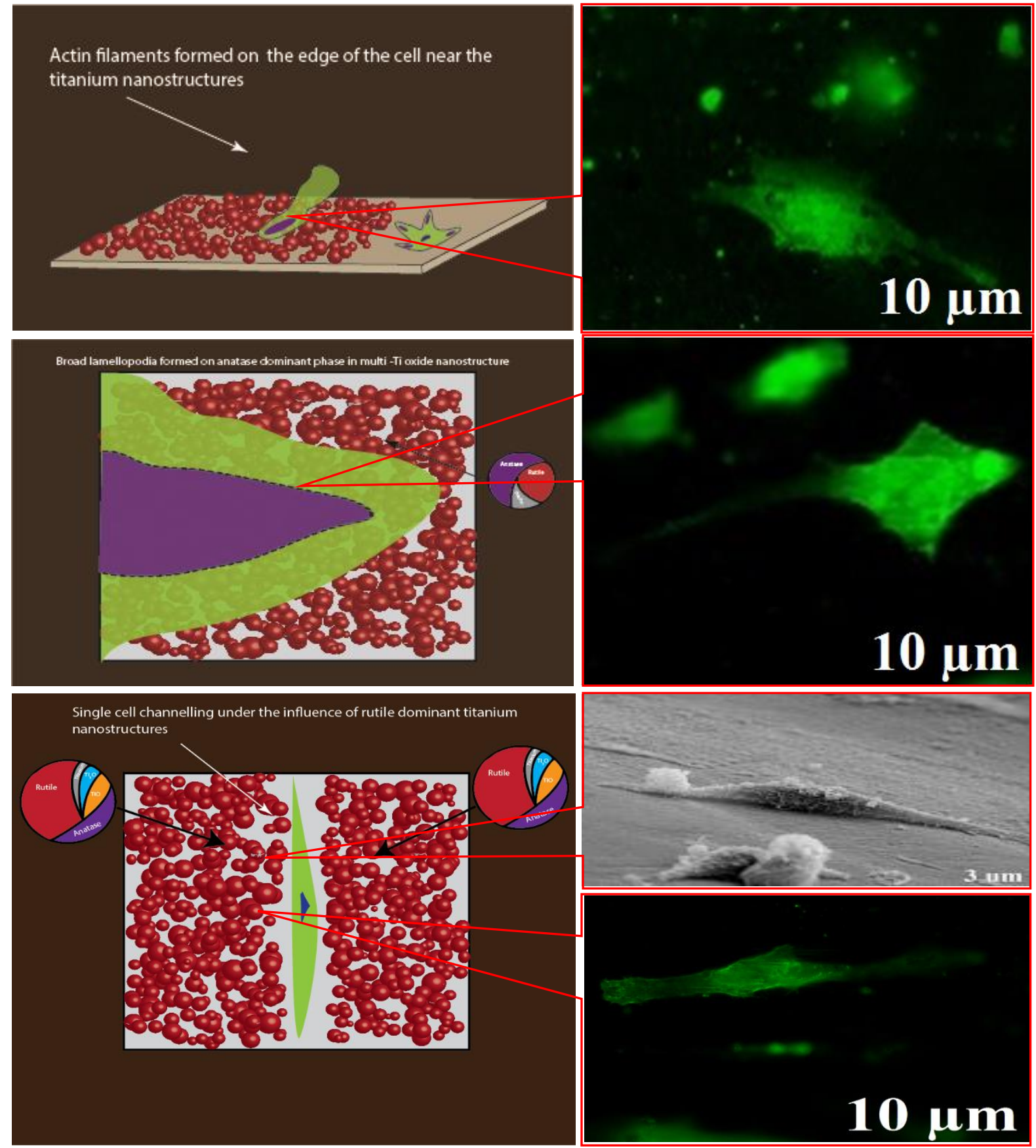


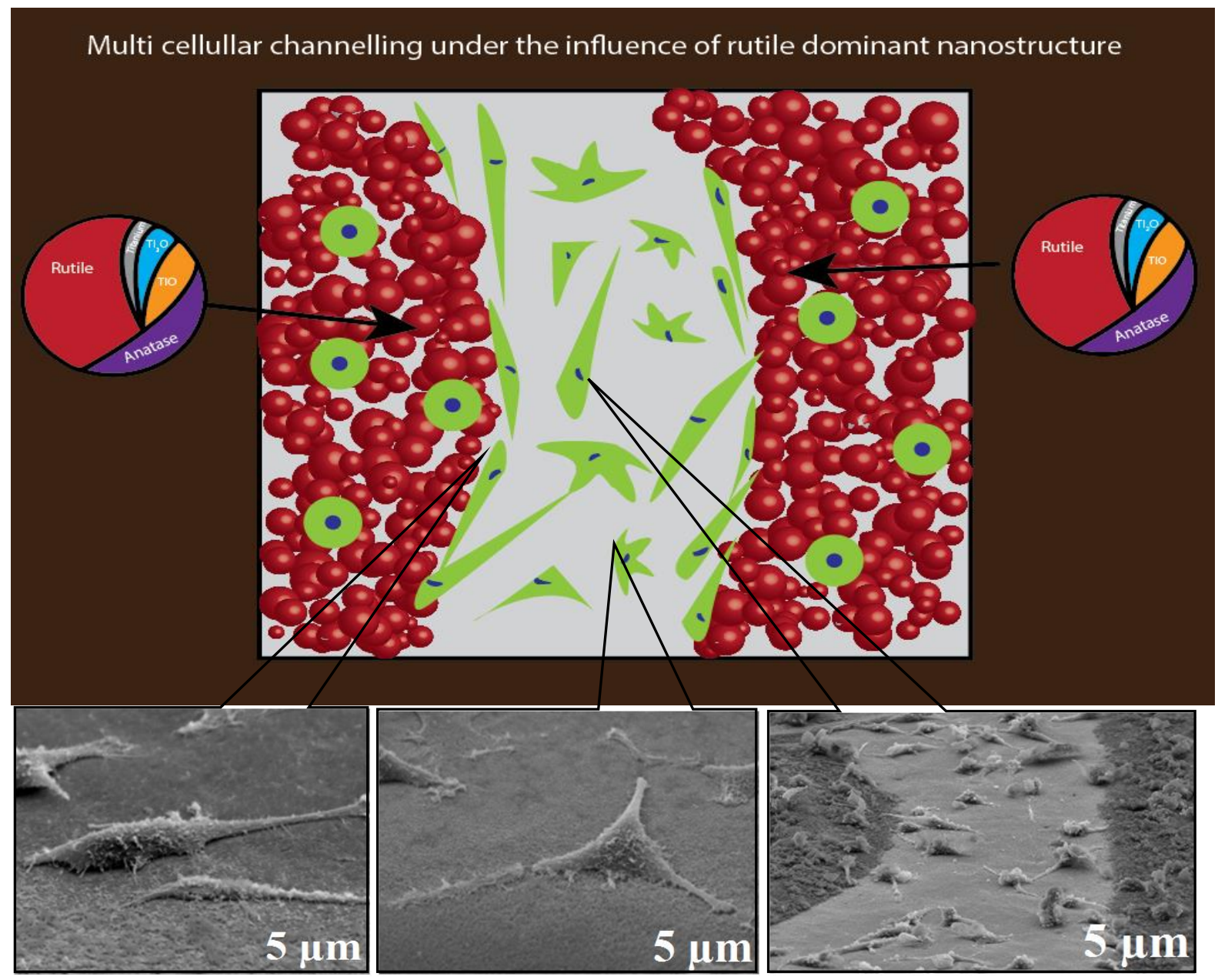

Figure 29: Influence of dominant phases of multi-Ti oxide nanostructure in multi cell channelling and single cell channelling

The cells appeared to randomly arranged but when they are near anatase dominant nanostructure they showed arrangement of aligning themselves parallel to the rutile dominant nanostructure, which indicate that these cells sense through haptotaxis mechanism where chemo gradient of the material was influenced by cancer cell. Thermodynamic nature of the nanostructure results in repelling of both the mammalian cells and also cancer cells away from the dominant phases of multi-Ti oxide nano structure when they come in contact and this phenomena is observed only when rutile and non-stoichiometric cubic $\mathrm{TiO}$ oxide dominant. The oxide variation in the nanostructure results in varied thermodynamic stress on the nano structure and these stresses when sensed by the cervical cancer cells, their morphology is reorganized by avoiding further interaction with the nanostructure. Schakenraad et al., stated that higher the surface energy lead to higher cell adhesion of HeLa cells. Thus it is suggested that at only 24 hours period the cancer cells moves away from the higher surface energy area i.e. rutile and non-stoichiometric cubic TiO oxide 
dominant. At figure 29, it is observed that the cells repel away when they approach the nanostructure and the filopodia attached to the cells also repelling away. But the nanostructure synthesized at high peak power at longer pulse to pulse width, broader lamellipodia is formed on the anatase dominant nanostructure which is not observed at high peak power shorter pulse to pulse width having rutile dominant nanostructure. Cell channelling is an interesting phenomenon where the influence of rutile dominance nanostructure resulted in needle shaped cell which was not achieved in anatase dominant nanostructure. Thus this cell characteristics is observed only due to the rutile and non-stoichiometric cubic $\mathrm{TiO}$ oxide phase present in the nanostructure. Broad lamellipodia of the cell is found from nanostructure is due to the activation of repulsion receptors and also due to the actin and microtubule dynamics are changed while simultaneously reducing adhesion between cells and the nano structure. However this influence of using anatase dominance nanostructure which indicate that this composition of phases of titanium oxides cannot be achieved by any other fabrication methods. Broad lamellipodia of the cell indicate that the cells favour to adhere and intend to proliferate. Needle shaped cytoskeleton behaviour of the cell intend to migrate away from the zone of the nanostructure they interact with.

\subsubsection{Effect of multi-Ti oxide phases of nanostructure for cancer cell directionality:}

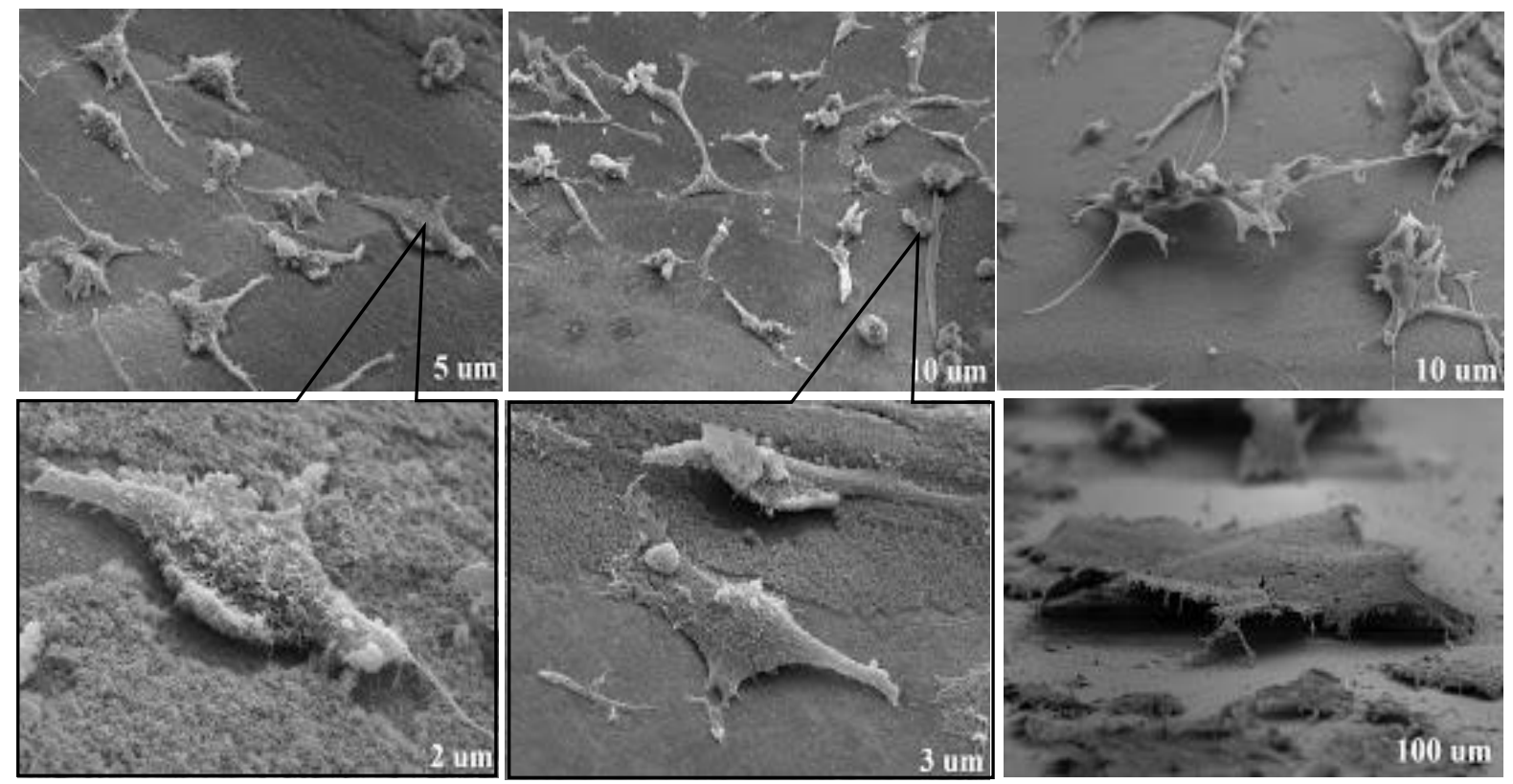

Figure 30: Cancer cell directionality is determined by the dominant phase of multi-Ti oxide nanostructure 
In figure 30, Cell trapping using the nano structures may be due to the activation of repulsion receptors present in the cells which is sensed through filopodia. Cell interaction with nanostructures is achieved through the formation of polarized "lamellipodia" and fine hair like protrusions termed "filopodia" which gather spatial, topographical and chemical information of the environment. Cell spreading is a series of process initiated from filopodia probe the environment followed by the establishment of focal adhesion of the cell and finally cell protrusions. Focal contact are formed at the leading edge of the migrating cell and often mature into focal adhesions. However the intracellular mechanism by which cell protrude is not fully understood. This intricate relationship between cancer cell and the multi-Ti oxide nanostructures is unique where the cell trapping phenomenon happens only when rutile dominant nanostructures interact resulting in cancer cells trapped and not allowing cells to migrate. This results indicate the cells signals to each other and the cells which make the first contact to nanostructure transmit the signals to nearby cells to avoid or migrate towards the nanostructure.

\subsubsection{HeLa cell alignment in the proliferative zone due to the influence of energy from the ultrashort pulsed laser:}

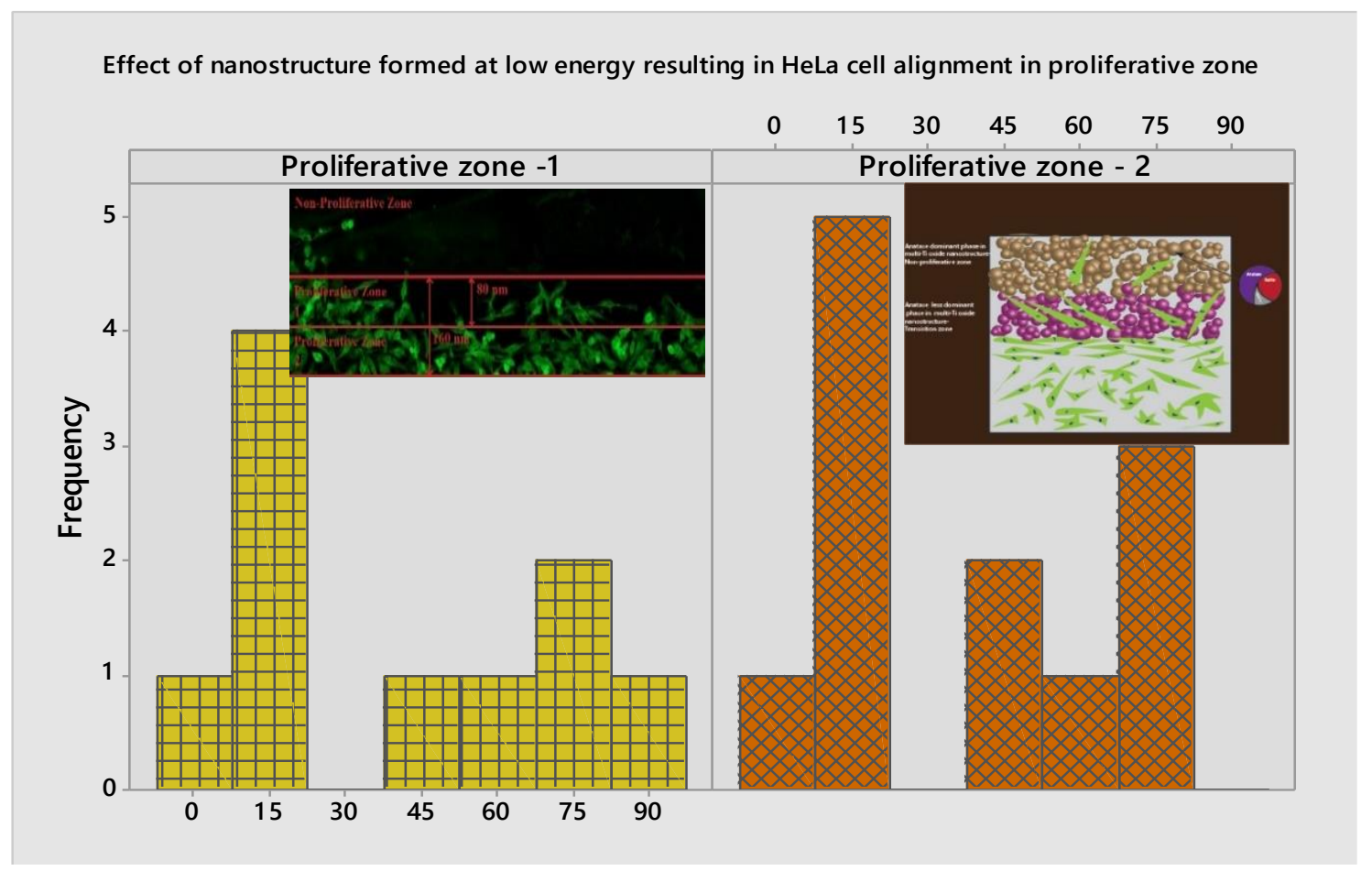


Figure 31: Influence of dominant phase of multi-Ti oxide phased nanostructure determines the HeLa cell alignment at 24 hours

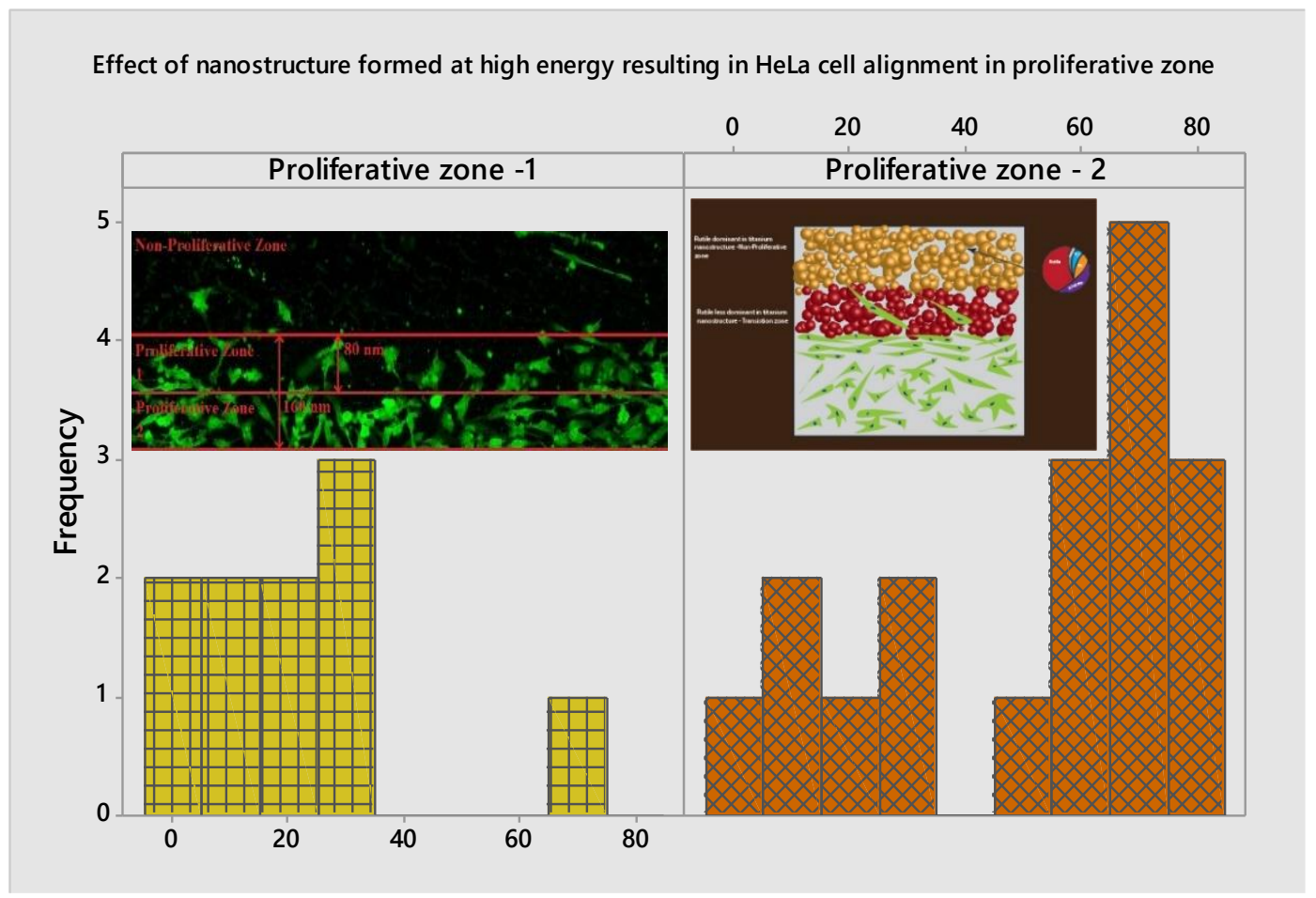

HeLa cancer cell adhesion on multi-Ti oxide nanostructures demosntrated adhesion complexes with clear alignment along the direction of the multi-Ti oxide phased nanostructure pattern. From the figure 28 , it is inferred that dominant phases present in the multi-Ti oxide nanostructure has influence in determining the alignment of cancer cells towards the nanostructure. However the degree of alignment of cancer cells depends the zones of nanostructure deposited onto the substrate. These zones are categorized based upon the number of cells aligned paralelly to the nanostructures. This indicate that at proliferative zone -1 there were no cells aligned vertically to the nanostructure which indicate defienetly that cells tend to move away. However in the proliferative zone -2 more cells are vertically aligned than cells horizontally which indicate the cells intend to move toward. Cells intially senses by aligning vertically and depending on the favourable area for them to adhere they move forward otherwise they move away from the nanostructure. This interesting phenomeno aids us in understanding the mechanism of cell interaction with the nanostructured biomaterial. 


\subsubsection{Rounding of HeLa cancer cell when interacting with multi-Ti oxide phased nanostructure:}
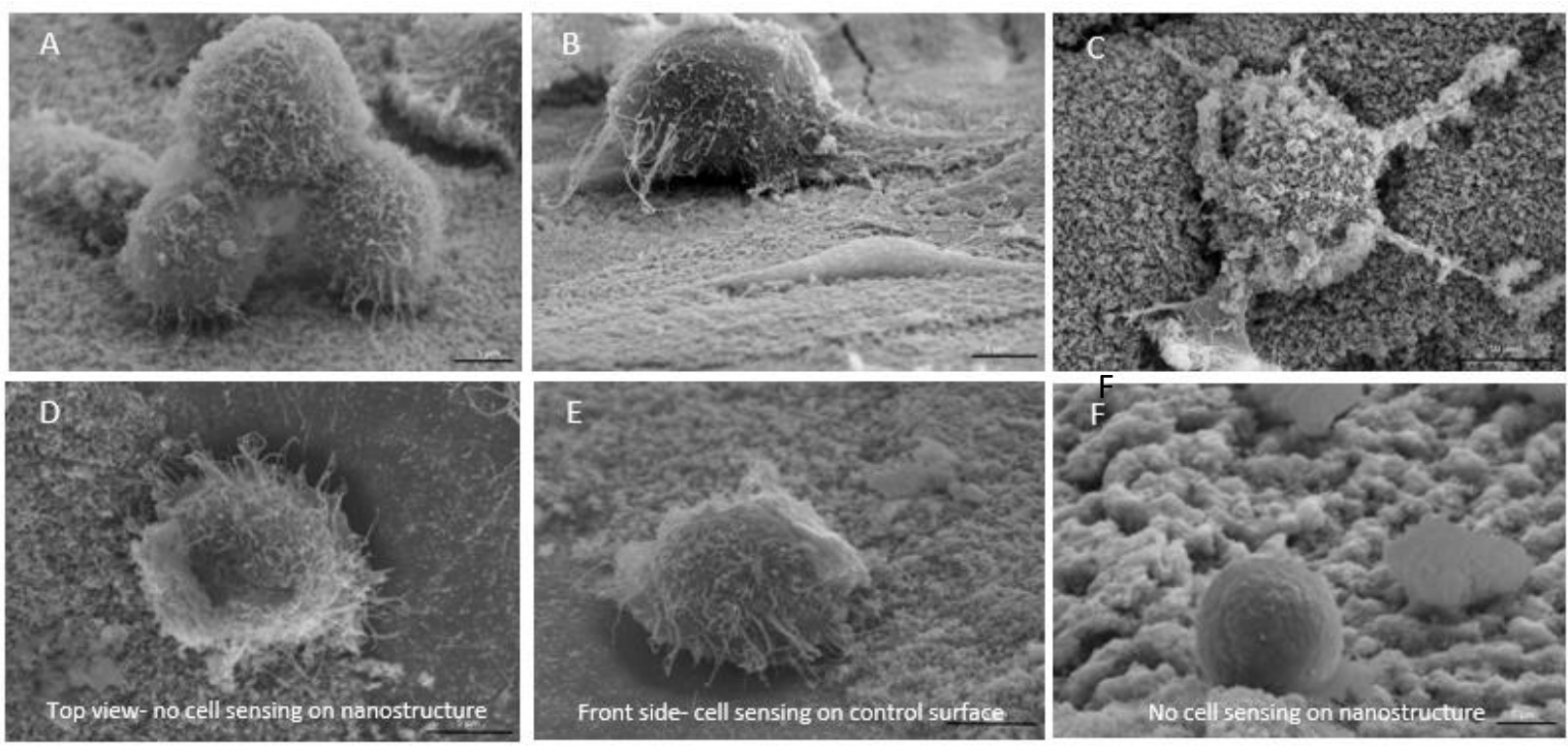

Figure 32: Scanning electron micrograph showing the influence of titanium nano material when interacting with HeLa on (A -C) Anatase dominant titanium nano material (D-F) Rutile and non-stoichiometric cubic TiO oxide dominant

We observed a distinct HeLa cell sensing ability to gather the information for it adhere and proliferate when they interacted with multi-Ti oxide phased nanostructure. In the above figure 32(A) and 32(B) it is observed that rounded cells when interact with anatase dominant titanium nano material they were able to adhere firmly producing lamellae and filopodia. Closer examination showed that filopodia are seen to interact mostly with nano material and at this point it is concluded that the anatase phase which is dominant in multi-Ti oxide phased nanostructure which is synthesized at low peak power at short pulse width is favored for cells to adhere and proliferate. But in the condition where rutile and non-stoichiometric cubic oxide is dominant in titanium nanomaterial it is clearly evident as per figure 32(D) and 32(E) that cells initiate filopodia formation only on plain titanium substrate but not on the nanomaterial. Cells which is located on multi-Ti oxide phased nanostructure as in figure 32(F) there is no formation of filopodia which is directly opposite to the cells interact with anatase dominant nanostructure. Thus this altered dynamics of cell spreading will eventually lead to cell death and leads to reduction in HeLa cancer cells in the presence of multi-Ti oxide phased nanostructure. 


\subsection{Conclusion:}

Engineering the cell-cell communication is essential in designing the cancer cell architecture surrounding the mammalian cells which would enable us to exactly manipulate the cancer cells. There have been no techniques available where cell-cell communication can be manipulated using the material composition of the nanostructure which would enable us to determine cell cytoskeleton behaviour. However currently cell-cell communication can be determined using nanostructured morphology. Interestingly, the phase dominance of titanium oxide nanostructure synthesized have provided evidence that they can determine the cell signaling which would enable us to understand the fundamental knowledge of cell-cell communication. 


\section{Chapter- 6 \\ Summary and future research work}

\subsection{Summary:}

In this thesis, a unique composition of multi-Ti oxide having dominance phases was synthesized using varying the ionization energy from ultrashort pulsed laser. Notable reduction in cancer cell proliferative is achieved when multi-Ti oxide nanostructures is interacted with the cancer cell. Also, in this thesis cardinal approach in studying the cancer cell cytoskeleton behaviour using multi-Ti oxide nanostructures is achieved, which has the application in designing biomaterial having ying-yang proliferative properties. Mammalian cell does not have the influence of dominant phases of titanium oxides when compared to the HeLa cancer cell which indicate that this unique combinatorial material composition of titanium oxides can selectively control their cytoskeleton behaviour. In an effort, multi-Ti oxide nanostructures not only able to modulate the cancer cell behaviour but when these nanostructures synthesized in a patterned manner proliferative and non-proliferative zones of the dominant phases of the titanium oxides results in controlling the HeLa cell but not mammalian cell. This approach of synthesizing ying -yang proliferative biomaterial has the application in implants, where mammalian cells has to proliferate but not the HeLa cancer cell. This would enable the patients after sparing surgery have a better quality of life instead having repetitive surgery because of reoccurrence of cancer.

\subsection{Recommended future work:}

This research work has promising prospects in array of applications such as tissue engineering and drug-delivery systems. The cell-biomaterial interaction properties can be further probed by tailoring the composition of titanium oxides for stem cell proliferative study. Therefore there is wide application using the multi-Ti oxide nanostructures for differentiation of stem cells into bone lineage. Further similar approach of controlling cancer cell proliferation can be applied to antibacterial applications. 


\section{References:}

1. Yang L, Liu H, Lin Y. Biomaterial nanotopography-mediated cell responses: experiment and modeling. Int J Smart Nano Mater. 2014;2014. doi:10.1080/19475411.2014.995744.

2. Karuri NW, Liliensiek S, Teixeira AI, et al. Biological length scale topography enhances cell-substratum adhesion of human corneal epithelial cells. J Cell Sci. 2004;117(Pt 15):3153-3164. doi:10.1242/jcs.01146.

3. Bacakova L, Filova E, Parizek M, Ruml T, Svorcik V. Modulation of cell adhesion, proliferation and differentiation on materials designed for body implants. Biotechnol Adv. 2011. doi:10.1016/j.biotechadv.2011.06.004.

4. Richert L, Vetrone F, Yi J-HH, et al. Surface Nanopatterning to Control Cell Growth. $A d v$ Mater. 2008;20(8):1488-1492. doi:10.1002/adma.200701428.

5. Jeon H. Biomaterials Design for Control of Cell Behavior by Femtosecond Laser Processing By Hojeong Jeon A dissertation submitted in partial satisfaction of the requirements for the degree of Doctor of Philosophy in Engineering - Mechanical Engineering in the Gra. 2011.

6. Yao X, Peng R, Ding J. Cell-material interactions revealed via material techniques of surface patterning. Adv Mater. 2013;25(37):5257-5286. doi:10.1002/adma.201301762.

7. Whitesides GM. The "right" size in nanobiotechnology. Nat Biotechnol. 2003;21(10):1161-1165. doi:10.1038/nbt872.

8. Minagar S, Wang J, Berndt CC, Ivanova EP, Wen C. Cell response of anodized nanotubes on titanium and titanium alloys. J Biomed Mater Res A. 2013;101(9):2726-2739. doi:10.1002/jbm.a.34575.

9. Kim Y, Muruganandham M, Modrick JM, Bayouth JE. Evaluation of artifacts and distortions of titanium applicators on 3.0-tesla MRI: Feasibility of titanium applicators in MRI-guided brachytherapy for gynecological cancer. Int J Radiat Oncol Biol Phys. 2011;80(3):947-955. doi:10.1016/j.ijrobp.2010.07.1981.

10. Park J, Bauer S, Mark K Von Der, Schmuki P. Nanosize and Vitality: TiO 2 Nanotube Diameter Directs Cell Fate. Nano Lett. 2007;7(6):1686-1691.

11. Lee J, Kang BS, Hicks B, et al. The control of cell adhesion and viability by zinc oxide nanorods. Biomaterials. 2008;29(27):3743-3749. doi:10.1016/j.biomaterials.2008.05.029.

12. Choi C-H, Hagvall SH, Wu BM, Dunn JCY, Beygui RE, CJ Kim C-J. Cell interaction with three-dimensional sharp-tip nanotopography. Biomaterials. 2007;28(9):1672-1679. doi:10.1016/j.biomaterials.2006.11.031. 
13. Girard PP, Cavalcanti-Adam E a., Kemkemer R, Spatz JP. Cellular chemomechanics at interfaces: sensing, integration and response. Soft Matter. 2007;3(3):307.

doi:10.1039/b614008d.

14. Petty RT, Li HW, Maduram JH, Ismagilov R, Mrksich M. Attachment of cells to islands presenting gradients of adhesion ligands. J Am Chem Soc. 2007;129(29):8966-8967. doi:10.1021/ja0735709.

15. Macak JM, Tsuchiya H, Ghicov a., et al. TiO2 nanotubes: Self-organized electrochemical formation, properties and applications. Curr Opin Solid State Mater Sci. 2007;11(1-2):318. doi:10.1016/j.cossms.2007.08.004.

16. Zwilling V, Aucouturier M, Darque-Ceretti E. Anodic oxidation of titanium and TA6V alloy in chromic media. An electrochemical approach. Electrochim Acta. 1999;45(6):921929. doi:10.1016/S0013-4686(99)00283-2.

17. Popat KC, Leoni L, Grimes C a., Desai $\mathrm{T}$ a. Influence of engineered titania nanotubular surfaces on bone cells. Biomaterials. 2007;28(21):3188-3197.

doi:10.1016/j.biomaterials.2007.03.020.

18. Ahn YC, Park SK, Kim GT, et al. Development of high efficiency nanofilters made of nanofibers. Curr Appl Phys. 2006;6(6 SPEC. ISS.):1030-1035. doi:10.1016/j.cap.2005.07.013.

19. Doshi J, Reneker DH. Electrospinning process and applications of electrospun fibers. Conf Rec 1993 IEEE Ind Appl Conf Twenty-Eighth IAS Annu Meet. 1993. doi:10.1109/IAS.1993.299067.

20. Zuwei Ma, Wei He, Thomas Yong SR. Grafting of gelatin on electrospun poly(caprolactone) nanofibers to improve endothelial cell spreading and proliferation and to control cell orientation. Tissue Eng. 2005;11(7).

21. Yang F, Murugan R, Wang S, Ramakrishna S. Electrospinning of nano/micro scale poly(llactic acid) aligned fibers and their potential in neural tissue engineering. Biomaterials. 2005;26(15):2603-2610. doi:10.1016/j.biomaterials.2004.06.051.

22. Stankus JJ, Guan J, Wagner WR. Fabrication of biodegradable elastomeric scaffolds with sub-micron morphologies. J Biomed Mater Res A. 2004;70(4):603-614. doi:10.1002/jbm.a.30122.

23. Ohgo K, Zhao C, Kobayashi M, Asakura T. Preparation of non-woven nanofibers of Bombyx mori silk, Samia cynthia ricini silk and recombinant hybrid silk with electrospinning method. Polymer (Guildf). 2002;44(3):841-846. doi:10.1016/S00323861(02)00819-4. 
24. Matthews J a, Boland ED, Wnek GE, Simpson DG, Bowlin GL. Electrospinning of collagen type 2 A feasibility study. J Bioact Compat Polym. 2003;18(March 2003):125134. doi:10.1177/088391103033856.

25. Um IC, Fang D, Hsiao BS, Okamoto A, Chu B. Electro-Spinning and Electro-Blowing of Hyaluronic Acid. Biomacromolecules. 2004;5:1428-1436.

26. Ma Z, Kotaki M, Ramakrishna S. Electrospun cellulose nanofiber as affinity membrane. $J$ Memb Sci. 2005;265(1-2):115-123. doi:10.1016/j.memsci.2005.04.044.

27. Chen Z, Mo X, Qing F. Electrospinning of collagen-chitosan complex. Mater Lett. 2007;61(16):3490-3494. doi:10.1016/j.matlet.2006.11.104.

28. Ekaputra AK, Prestwich GD, Cool SM, Hutmacher DW. Combining electrospun scaffolds with electrosprayed hydrogels leads to three-dimensional cellularization of hybrid constructs. Biomacromolecules. 2008;9(8):2097-2103. doi:10.1021/bm800565u.

29. Bhardwaj N, Kundu SC. Electrospinning: A fascinating fiber fabrication technique. Biotechnol Adv. 2010;28(3):325-347. doi:10.1016/j.biotechadv.2010.01.004.

30. Couleau N, Techer D, Pagnout C, et al. Hemocyte responses of Dreissena polymorpha following a short-term in vivo exposure to titanium dioxide nanoparticles: Preliminary investigations. Sci Total Environ. 2012;438:490-497. doi:10.1016/j.scitotenv.2012.08.095.

31. Jacobasch C, Völker C, Giebner S, et al. Long-term effects of nanoscaled titanium dioxide on the cladoceran Daphnia magna over six generations. Environ Pollut. 2014;186:180186. doi:10.1016/j.envpol.2013.12.008.

32. Frenzilli G, Bernardeschi M, Guidi P, et al. Effects of in vitro exposure to titanium dioxide on DNA integrity of bottlenose dolphin (Tursiops truncatus) fibroblasts and leukocytes. Mar Environ Res. 2014;100:68-73. doi:10.1016/j.marenvres.2014.01.002.

33. Divya Rani V V., Vinoth-Kumar L, Anitha VC, Manzoor K, Deepthy M, Shantikumar VN. Osteointegration of titanium implant is sensitive to specific nanostructure morphology. Acta Biomater. 2012;8(5):1976-1989. doi:10.1016/j.actbio.2012.01.021.

34. Li Z, Pan X, Wang T, Wang P-N, Chen J-Y, Mi L. Comparison of the killing effects between nitrogen-doped and pure $\mathrm{TiO} 2$ on HeLa cells with visible light irradiation. Nanoscale Res Lett. 2013;8(1):96. doi:10.1186/1556-276X-8-96.

35. Yin ZF, Wu L, Yang HG, Su YH. Recent progress in biomedical applications of titanium dioxide. Phys Chem Chem Phys. 2013;15(14):4844-4858. doi:10.1039/c3cp43938k.

36. Mullick Chowdhury S, Lalwani G, Zhang K, Yang JY, Neville K, Sitharaman B. Cell specific cytotoxicity and uptake of graphene nanoribbons. Biomaterials. 2013;34(1):283293. doi:10.1016/j.biomaterials.2012.09.057. 
37. Tavangar A. FEMTOSECOND LASER NANO-FABRICATION AND ITS BIOMEDICAL APPLICATIONS by.; 2013.

38. Bass JD, Belamie E, Grosso D, Boissiere C, Coradin T, Sanchez C. Nanostructuration of titania films prepared by self-assembly to affect cell adhesion. J Biomed Mater Res A. 2010;93(1):96-106. doi:10.1002/jbm.a.32477.

39. Andersson A, Brink J, Lidberg U, Sutherland DS. Influence of systematically varied nanoscale topography on the morphology of epithelial cells. IEEE Trans Nanobioscience. 2003;2(2):49-57. doi:10.1109/TNB.2003.813934.

40. Diehl K a, Foley JD, Nealey PF, Murphy CJ. Nanoscale topography modulates corneal epithelial cell migration. J Biomed Mater Res A. 2005;75(3):603-611. doi:10.1002/jbm.a.30467.

41. Yim EKF, Leong KW. Significance of synthetic nanostructures in dictating cellular response. Nanomedicine. 2005;1(1):10-21. doi:10.1016/j.nano.2004.11.008.

42. Geiger B, Spatz JP, Bershadsky AD. Environmental sensing through focal adhesions. Nat Rev Mol Cell Biol. 2009;10(1):21-33. doi:10.1038/nrm2593.

43. Vogel V, Sheetz M. Local force and geometry sensing regulate cell functions. Nat Rev Mol Cell Biol. 2006;7(4):265-275. doi:10.1038/nrm1890.

44. Curtis ASG, Wilkinson CDW. Reactions of cells to topography. J Biomater Sci Polym Educ. 1998;9(12):1313-1329.

45. Anselme K, Davidson P, Popa a M, Giazzon M, Liley M, Ploux L. The interaction of cells and bacteria with surfaces structured at the nanometre scale. Acta Biomater. 2010;6(10):3824-3846. doi:10.1016/j.actbio.2010.04.001.

46. Biggs MJP, Richards RG, Dalby MJ. Nanotopographical modification: a regulator of cellular function through focal adhesions. Nanomedicine. 2010;6(5):619-633. doi:10.1016/j.nano.2010.01.009.

47. Kim D, Ghicov A, Schmuki P. TiO2 Nanotube arrays: Elimination of disordered top layers ("nanograss") for improved photoconversion efficiency in dye-sensitized solar cells. Electrochem commun. 2008;10(12):1835-1838. doi:10.1016/j.elecom.2008.09.029.

48. Zhang Y, Xiang Q, Dong S, Li C, Zhou Y. Fabrication and characterization of a recombinant fibronectin/cadherin bio-inspired ceramic surface and its influence on adhesion and ossification in vitro. Acta Biomater. 2010;6(3):776-785. doi:10.1016/j.actbio.2009.08.025. 
49. Park JS, Yang HN, Woo DG, Jeon SY, Park KH. Chondrogenesis of human mesenchymal stem cells in fibrin constructs evaluated in vitro and in nude mouse and rabbit defects models. Biomaterials. 2011;32(6):1495-1507. doi:10.1016/j.biomaterials.2010.11.003.

50. Macdonald ML, Samuel RE, Shah NJ, Padera RF, Beben YM, Hammond PT. Tissue integration of growth factor-eluting layer-by-layer polyelectrolyte multilayer coated implants. Biomaterials. 2011;32(5):1446-1453. doi:10.1016/j.biomaterials.2010.10.052.

51. Daoud W a., Xin JH, Zhang YH. Surface functionalization of cellulose fibers with titanium dioxide nanoparticles and their combined bactericidal activities. Surf Sci. 2005;599(1-3):69-75. doi:10.1016/j.susc.2005.09.038.

52. Kazemzadeh-Narbat M, Lai BFL, Ding C, Kizhakkedathu JN, Hancock REW, Wang R. Multilayered coating on titanium for controlled release of antimicrobial peptides for the prevention of implant-associated infections. Biomaterials. 2013;34(24):5969-5977. doi:10.1016/j.biomaterials.2013.04.036.

53. $\mathrm{Hu}$ Y, Cai K, Luo Z, et al. TiO 2 nanotubes as drug nanoreservoirs for the regulation of mobility and differentiation of mesenchymal stem cells. Acta Biomater. 2012;8(1):439448. doi:10.1016/j.actbio.2011.10.021.

54. Huo K, Zhang X, Wang H, Zhao L, Liu X, Chu PK. Osteogenic activity and antibacterial effects on titanium surfaces modified with $\mathrm{Zn}$-incorporated nanotube arrays. Biomaterials. 2013;34(13):3467-3478. doi:10.1016/j.biomaterials.2013.01.071.

55. Park J, Bauer S, Schlegel KA, Neukam FW, von der Mark K, Schmuki P. TiO2 nanotube surfaces: $15 \mathrm{~nm}$--an optimal length scale of surface topography for cell adhesion and differentiation. Small. 2009;5(6):666-671. doi:10.1002/smll.200801476.

56. Tillack MS, Blair DW, Harilal SS. The effect of ionization on cluster formation in laser ablation plumes. Nanotechnology. 2004;15:390-403. doi:10.1088/0957-4484/15/3/028.

57. Gamaly EG, Madsen NR, Golberg D, Rode a. V. Expansion-limited aggregation of nanoclusters in a single-pulse laser-produced plume. Phys Rev B. 2009;80(18):184113. doi:10.1103/PhysRevB.80.184113.

58. Tan B, Venkatakrishnan K. Synthesis of fibrous nanoparticle aggregates by femtosecond laser ablation in air. Opt Express. 2009;17(2):1064-1069. doi:10.1364/OE.17.001064.

59. Tavangar A, Tan B, Venkatakrishnan K. Synthesis of bio-functionalized threedimensional titania nanofibrous structures using femtosecond laser ablation. Acta Biomater. 2011;7(6):2726-2732. doi:10.1016/j.actbio.2011.02.020.

60. Duscn MS Van, Kingery WD, Laubitz MJ, et al. Kinetics of the Anatase-Rutile Transformation. J Am Ceram Soc. 1965;(August). 
61. Zhang H, Banfield JF. Understanding Polymorphic Phase Transformation Behavior during Growth of Nanocrystalline Aggregates: Insights from TiO2. J Phys Chem B.

2000;104(15):3481-3487. doi:10.1021/jp000499j.

62. Gouma PI, Mills MJ. Anatase-to-Rutile Transformation in Titania Powders. J Am Ceram Soc. 2001;84(189042):619-622. doi:10.1111/j.1151-2916.2001.tb00709.x.

63. Banerjee I, Karmakar S, Kulkarni N V., et al. Effect of ambient pressure on the crystalline phase of nano $\mathrm{TiO} 2$ particles synthesized by a dc thermal plasma reactor. J Nanoparticle Res. 2009;12(2):581-590. doi:10.1007/s11051-009-9627-9.

64. Harano A, Shimada K, Okubo T, Sadakata M. Crystal phases of TiO 2 ultrafine particles prepared by laser ablation of solid rods. J Nanoparticle Res. 2002;(3):215-219.

65. Ingber DE. Tensegrity I. Cell structure and hierarchical systems biology. J Cell Sci. 2003;116(7):1157-1173. doi:10.1242/jcs.00359.

66. Lu J, Rao MP, MacDonald NC, Khang D, Webster TJ. Improved endothelial cell adhesion and proliferation on patterned titanium surfaces with rationally designed, micrometer to nanometer features. Acta Biomater. 2008;4:192-201. doi:10.1016/j.actbio.2007.07.008.

67. Yu WQ, Jiang XQ, Zhang FQ, Xu L. The effect of anatase TiO2 nanotube layers on MC3T3-E1 preosteoblast adhesion, proliferation, and differentiation. J Biomed Mater Res - Part A. 2010;94(4):1012-1022. doi:10.1002/jbm.a.32687.

68. Yu WQ, Zhang YL, Jiang XQ, Zhang FQ. In vitro behavior of MC3T3-E1 preosteoblast with different annealing temperature titania nanotubes. Oral Dis. 2010;16(7):624-630. doi:10.1111/j.1601-0825.2009.01643.x.

69. Lee J, Chu BH, Chen K-H, Ren F, Lele TP. Randomly oriented, upright SiO2 coated nanorods for reduced adhesion of mammalian cells. Biomaterials. 2009;30(27):44884493. doi:10.1016/j.biomaterials.2009.05.028.

70. Re F, Zanetti A, Sironi M, et al. Inhibition of Anchorage-dependent Cell Spreading Triggers Apoptosis in Cultured Human Endothelial Cells. J Cell Biol. 1994;127(2):537546.

71. Csaderova L, Martines E, Seunarine K, Gadegaard N, Wilkinson CDW, Riehle MO. A biodegradable and biocompatible regular nanopattern for large-scale selective cell growth. Small. 2010;6(23):2755-2761. doi:10.1002/smll.201000193.

72. Schiele NR, Corr DT, Huang Y, Raof NA, Xie Y, Chrisey DB. Laser-based direct-write techniques for cell printing. Biofabrication. 2010;2(3):032001. doi:10.1088/17585082/2/3/032001. 
73. Kohn J, Welsh WJ, Knight D. A new approach to the rationale discovery of polymeric biomaterials. Biomaterials. 2007;28(29):4171-4177.

doi:10.1016/j.biomaterials.2007.06.022.

74. Simon CG, Stephens JS, Dorsey SM, Becker ML. Fabrication of combinatorial polymer scaffold libraries. Rev Sci Instrum. 2007;78(7). doi:10.1063/1.2755761.

75. Cukierman E, Pankov R, Yamada KM. Cell interactions with three-dimensional matrices. Curr Opin Cell Biol. 2002;14(5):633-639. doi:10.1016/S0955-0674(02)00364-2.

76. Cukierman E, Pankov R, Stevens DR, Yamada KM. Taking cell-matrix adhesions to the third dimension. Science. 2001;294(5547):1708-1712. doi:10.1126/science.1064829.

77. Walboomers XF, Croes HJE, Ginsel L a., Jansen J a. Growth behavior of fibroblasts on microgrooved polystyrene. Biomaterials. 1998;19(20):1861-1868. doi:10.1016/S01429612(98)00093-3.

78. Clarke P, Connolly P. Topographical control of cell behaviour : 2. Multiple grooved substrata. 1990;644:635-644. http://strathprints.strath.ac.uk/28851/.

79. Zinchenko YS, Schrum LW, Clemens M, Coger RN. Hepatocyte and kupffer cells cocultured on micropatterned surfaces to optimize hepatocyte function. Tissue Eng. 2006;12(4):751-761. doi:10.1089/ten.2006.12.751.

80. Folch A, Jo BH, Hurtado O, Beebe DJ, Toner M. Microfabricated elastomeric stencils for micropatterning cell cultures. J Biomed Mater Res. 2000;52(2):346-353. doi:10.1002/1097-4636(200011)52:2<346::AID-JBM14>3.0.CO;2-H.

81. Rohr S, Flückiger-Labrada R, Kucera JP. Photolithographically defined deposition of attachment factors as a versatile method for patterning the growth of different cell types in culture. Pflugers Arch. 2003;446(1):125-132. doi:10.1007/s00424-002-1000-0.

82. Ginger DS, Zhang H, Mirkin C a. The Evolution of Dip-Pen Nanolithography. Angew Chemie - Int Ed. 2004;43(1):30-45. doi:10.1002/anie.200300608.

83. Roth E a., Xu T, Das M, Gregory C, Hickman JJ, Boland T. Inkjet printing for highthroughput cell patterning. Biomaterials. 2004;25(17):3707-3715. doi:10.1016/j.biomaterials.2003.10.052.

84. Powell HM, Kniss DA, Lannutti JJ. Nanotopographic Control of Cytoskeletal Organization. Langmuir. 2006;22(11):5087-5094.

85. Dalby MJ, Yarwood SJ, Riehle MO, Johnstone HJH, Affrossman S, Curtis ASG. Increasing fibroblast response to materials using nanotopography: morphological and genetic measurements of cell response to 13-nm-high polymer demixed islands. Exp Cell Res. 2002;276(1):1-9. doi:10.1006/excr.2002.5498. 
86. Brock A, Chang E, Ho CC, et al. Geometric determinants of directional cell motility revealed using microcontact printing. Langmuir. 2003;19(5):1611-1617.

doi:10.1021/la026394k.

87. Huang S. Gene expression profiling, genetic networks, and cellular states: An integrating concept for tumorigenesis and drug discovery. J Mol Med. 1999;77(6):469-480. doi:10.1007/s001099900023.

88. Molly M, Julian H. Exploring and Engineering the Cell Surface Interface. 2005.

89. Burridge K, Chrzanowska-Wodnicka M. Focal adhesions, contractility, and signaling. Annu Rev Cell Dev Biol. 1996;12:463-518. doi:10.1146/annurev.cellbio.12.1.463.

90. Wood W, Martin P. Structures in focus-filopodia. Int J Biochem Cell Biol. 2002;34(7):726-730. doi:10.1016/S1357-2725(01)00172-8.

91. Schmitz a a, Govek EE, Böttner B, Van Aelst L. Rho GTPases: signaling, migration, and invasion. Exp Cell Res. 2000;261(1):1-12. doi:10.1006/excr.2000.5049.

92. Jones GE. The Rho GTPases in Macrophage Motility and Chemotaxis. Cell Adhes Commun. 1998;6(2-3):237-245.

93. Kunzler TP, Huwiler C, Drobek T, Vörös J, Spencer ND. Systematic study of osteoblast response to nanotopography by means of nanoparticle-density gradients. Biomaterials. 2007;28(33):5000-5006. doi:10.1016/j.biomaterials.2007.08.009.

94. Efremov AN, Stanganello E, Welle A, Scholpp S, Levkin PA. Micropatterned superhydrophobic structures for the simultaneous culture of multiple cell types and the study of cell-cell communication. Biomaterials. 2013. doi:10.1016/j.biomaterials.2012.11.034.

95. Dalby MJ, Gadegaard N, Herzyk P, Agheli H, Sutherland DS, Wilkinson CDW. Group analysis of regulation of fibroblast genome on low-adhesion nanostructures. Biomaterials. 2007;28(10):1761-1769. doi:10.1016/j.biomaterials.2006.11.049.

96. Kim D-H, Han K, Gupta K, Kwon KW, Suh K-Y, Levchenko A. Mechanosensitivity of fibroblast cell shape and movement to anisotropic substratum topography gradients. Biomaterials. 2009;30(29):5433-5444. doi:10.1016/j.biomaterials.2009.06.042.

97. Arnold M, Hirschfeld-Warneken VC, Lohmüller T, et al. Induction of cell polarization and migration by a gradient of nanoscale variations in adhesive ligand spacing. Nano Lett. 2008;8(7):2063-2069. doi:10.1021/nl801483w. 